NASA CONTRACTOR REPORT

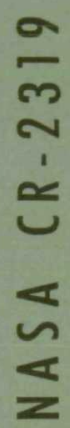
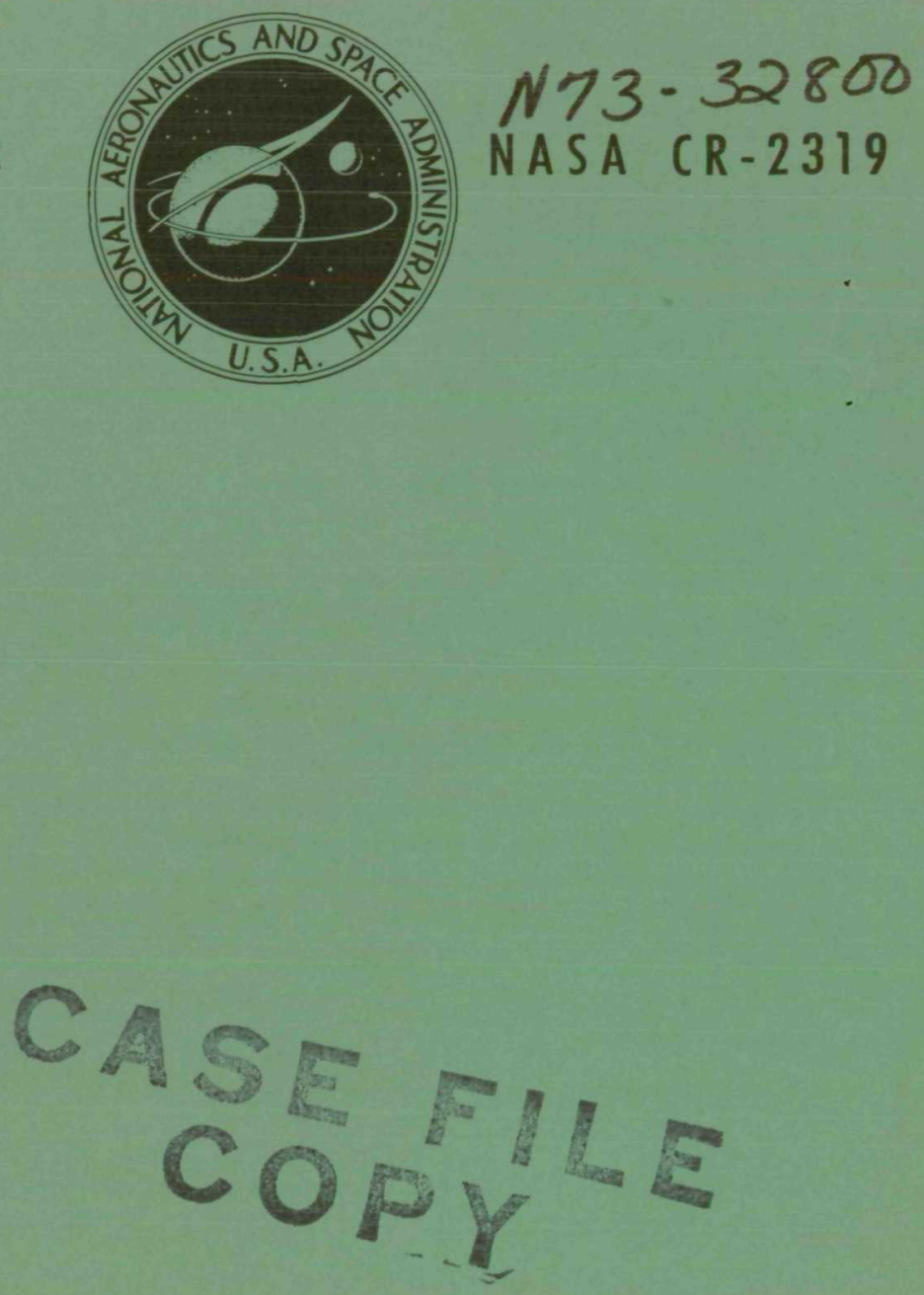

\title{
A STUDY OF \\ CRACK CLOSURE IN FATIGUE
}

by T. T. Shib and R. P. Wei

Prepared by

LEHIGH UNIVERSITY

Bethlehem, $\mathrm{Pa}$.

for Langley Research Center

NATIONAL AERONAUTICS AND SPACE ADMINISTRATION • WASHINGTON, D. C. • OCTOBER 1973 


\begin{tabular}{|c|c|c|c|}
\hline $\begin{array}{l}\text { 1. Report No. } \\
\text { NASA CR-2319 }\end{array}$ & 2. Government Accession No. & \multicolumn{2}{|c|}{ 3. Recipient's Catalog No. } \\
\hline \multirow{2}{*}{\multicolumn{2}{|c|}{$\begin{array}{l}\text { 4. Title and Subtitle } \\
\text { A STUDY OF CRACK CLOSU }\end{array}$}} & \multicolumn{2}{|c|}{$\begin{array}{l}\text { 5. Report Date } \\
\text { October } 1973\end{array}$} \\
\hline & & \multicolumn{2}{|c|}{$\begin{array}{l}\text { 6. Performing Organization Code } \\
\text {. }\end{array}$} \\
\hline \multicolumn{2}{|l|}{ 7. Author(s) } & \multicolumn{2}{|c|}{$\begin{array}{l}\text { 8. Performing Organization Report No. } \\
\text { IFSM-72-25 }\end{array}$} \\
\hline \multirow{2}{*}{\multicolumn{2}{|c|}{$\begin{array}{l}\text { 9. Performing Organization Name and Address } \\
\text { Department of Mechanical Engineering and Mechanics } \\
\text { Lehigh University } \\
\text { Bethlehem, PA }\end{array}$}} & \multicolumn{2}{|c|}{$\begin{array}{l}\text { 10. Work Unit No. } \\
501-22-02-01\end{array}$} \\
\hline & & \multicolumn{2}{|c|}{$\begin{array}{l}\text { 11. Contract or Grant No. } \\
\text { NGL-39-007-040 }\end{array}$} \\
\hline \multirow{2}{*}{\multicolumn{2}{|c|}{$\begin{array}{l}\text { 12. Sponsoring Agency Name and Address } \\
\text { National Aeronautics and Space Administration } \\
\text { Washington, D.C. } 20546\end{array}$}} & \multicolumn{2}{|c|}{$\begin{array}{l}\text { 13. Type of Report and Period Covered } \\
\text { Contractor Report }\end{array}$} \\
\hline & & \multicolumn{2}{|c|}{ 14. Sponsoring Agency Code } \\
\hline \multicolumn{4}{|c|}{$\begin{array}{l}\text { 15. Supplementary Notes } \\
\text { This is a final report. }\end{array}$} \\
\hline \multicolumn{4}{|c|}{$\begin{array}{l}\text { 16. Abstract } \\
\text { Crack closure phenomenon in fatigue was studied by using a Ti-6Al-4V titanium alloy. The } \\
\text { occurrence of crack closure was directly measured by an electrical-potential method, and indirectly } \\
\text { by load-strain measurement. The experimental results showed that the onset of crack closure } \\
\text { depends on both the stress ratio, } R \text {, and the maximum stress intensity factor, } K \text { max No crack } \\
\text { closure was observed for stress ratio, } R \text {, greater than } 0.3 \text { in this alloy. } \\
\text { A two-dimensional elastic model was used to explain the behavior of the recorded load-strain } \\
\text { curves. Closure force was estimated by using this model. Yield level stress was found near the } \\
\text { crack tip. Based on this estimated closure force, the crack opening displacement was calculated. } \\
\text { This result showed that onset of crack closure detected by electrical-potential measurement and } \\
\text { crack-opening-displacement measurement is the same. } \\
\text { The implications of crack closure on fatigue crack are considered. The experimental results } \\
\text { show that crack closure cannot fully account for the effect of stress ratio, } R \text {, on crack growth, } \\
\text { and that it cannot be regarded as the sole cause for delay. }\end{array}$} \\
\hline $\begin{array}{l}\text { 17. Key Words (Suggested by Autt } \\
\text { Fatigue crack growth } \\
\text { Crack closure } \\
\text { Ti-6Al-4V titanium all }\end{array}$ & $\begin{array}{l}\text { 18. Distrib } \\
\text { Unc lass }\end{array}$ & limited & \\
\hline $\begin{array}{l}\text { 19. Security Classif. (of this report) } \\
\text { Unclassified }\end{array}$ & $\begin{array}{l}\text { 20. Security Classif. (of this page) } \\
\text { Unclassified }\end{array}$ & $\begin{array}{l}\text { 21. No. of Pages } \\
85 \\
\end{array}$ & \begin{tabular}{|l} 
22. Price* \\
Domestic, $\$ 3.75$ \\
Foreign, $\$ 6.25$ \\
\end{tabular} \\
\hline
\end{tabular}

"For sale by the National Technical Information Service, Springfield, Virginia 22151 
TABLE OF CONTENTS

$\underline{\text { Page }}$

List of Tables

iv

List of Figures

$\mathrm{V}$

Summary

1

List of Symbols 2

I. INTRODUCTION

II. MATERIAL AND EXPERIMENTAL WORK

A. Material and Specimen $\quad 8$

B. Crack Monitoring System 9

C. Environment Control System 11

D. Experimental Work 11

1. Electrical Potential Measurements $\quad 12$

2. Strain Measurements $\quad 13$

III. RESULTS AND DISCUSSION 14

A. Electrical Potential Measurements 14

B. Strain Measurements $\quad 16$

C. The Effects of Stress Ratio and $\mathrm{K}_{\max }$ on Crack Closure 18

D. Discussion 19

IV. MODELING FOR CRACK CLOSURE 21

A. Analytical Model 21

B. Behavior of Load-Strain Curves 24

1. Strain Gage Located Ahead of the Crack Tip 24

2. Strain Gage Located Just Behind the Crack Tip 25

3. Strain Gage Located Behind the Crack Tip 26

C. Estimate of Closure Force $\quad 27$

D. Computation of Crack Opening Displacement 28

V. GENERAL DISCUSSION 29

VI. CONCLUSIONS

TABLES

FIGURES $\quad 36$

$\begin{array}{ll}\text { REFERENCES } & 74\end{array}$

$\begin{array}{ll}\text { APPENDIX } & 77\end{array}$ 


\section{LIST OF TABLES}

\section{Page}

TABLE I Chemical Composition and Tensile Properties 33

TABLE II Strain Gage Position 34

TABLE III Estimated Closure Stress

35 


\section{LIST OF FIGURES}

Figure

Page

1 Schematic Illustration of Load versus Crack-Opening

Displacement Curve

2 Center-Cracked Specimen $\quad 37$

3 Potential Leads on a Center-Cracked Specimen

4 Schematic Diagram of Electrical-Potential System for

Crack Measurement

5 Schematic Diagram of Environmental Control System

6 Load versus Electrical Potential Curves (Before the Compressive Loading Cycle)

$7 \quad$ Load versus Electrical Potential Curves (for the Compressive Loading Cycle)

8 Load versus Electrical Potential Curves (after the Compressive Loading Cycle)

9 Load versus Electrical Potential Curve for an Idealized Crack in an Elastic Medium

10 Load versus Strain Curve for an Idealized Crack in an Elastic Medium

12 Relationship between $U$ and $K_{\max }$ for Different Stress Ratios

14 Central Crack of Length $2 a$ in an Infinite Plate with Equal

Pairs of Splitting Forces at $\mathrm{x}= \pm \mathrm{b}$

15 Relationships between Strains and the Position of Unit Splitting Forces with Strain Gages Located Ahead of the Crack Tip $(\mathrm{a}=0.34 \mathrm{in})$

16 Relationships between Strains and the Position of Unit Splitting Forces with Strain Gage Located Behind the Crack Tip $(\mathrm{a}=0.908 \mathrm{in})$

17 Typical Load versus Strain Curve for a Strain Gage Located Ahead of the Crack Tip

18 Typical Load versus Strain Curve for a Strain Gage

Located Just Behind the Crack Tip

19 Longitudinal Strain Calculated by the Superposition

Model and the Estimated Closure Force 
20 Comparison of Probable Closure Line with Closure Line Indicated by the Electrical Potential Measurements

21 The Difference between the Plastic Zone Size Near the Surface and in the Center

22 Relationship between Load and Relative Displacement Between Gage Points Computed by Using the Superposition Model

23 Schematic Shown of the Onset of Crack Closure Detected by Three Different Experimental Techniques

24 Schematic Diagram Illustrating Effective $\Delta K$ for Several Cases

25 Crack Growth Rate for Ti-6AI-4V Alloy

26 The Effect of $K_{\min }$ on Delay 


\section{A STUDY OF CRACK CLOSURE IN FATIGUE}

by

T. T. Shih and R. P. Wei

LEHIGH UNIVERSITY

Bethlehem, Pennsylvania

\section{SUMMARY}

Crack closure phenomenon in fatigue was studied by using a Ti-6Al-4V titanium alloy. The occurrence of crack closure was directly measured by an electrical-potential method, and indirectly by load-strain measurement. The experimental results showed that the onset of crack closure depends on both the stress ratio, $R$, and the maximum stress intensity factor, $K_{\max }$. No crack closure was observed for stress ratio, $R$, greater than 0.3 in this alloy.

A two-dimensional elastic model. was used to explain the behavior of the recorded load-strain curves. Closure force was estimated by using this model. Yield level stress was found near the crack tip. Based on this estimated closure force, the crack opening displacement was calculated. This result showed that onset of crack closure detected by electrical-potential measurement and crack-opening-displacement measurement is the same.

The implications of crack closure on fatigue crack are considered. The experimental results show that crack closure cannot fully account for the effect of stress ratio, $R$, on crack growth, and that it cannot be regarded as the sole cause for delay. 


\section{LIST OF SYMBOLS}

a

$a_{\text {close }}$

$a_{0}$

$\triangle \mathrm{a} / \Delta \mathrm{N}$ rate of fatigue crack growth

$\mathrm{A}$

b

B

C

E

f (b)

$\mathrm{K}$

$\mathrm{K}_{\mathrm{c}}$

$\mathrm{K}_{\max }$

$\mathrm{K}_{\text {mean }}$

$\mathrm{K}_{\mathrm{min}}$

$\triangle \mathrm{K}$

$\triangle \mathrm{K}_{\mathrm{eff}}$

$\mathbf{n}$

$\mathrm{P}$

$\mathrm{P}_{\max }$

$P_{\min }$

$\mathrm{P}_{\text {op }}$

$\mathrm{R}$

$\mathrm{S}$

$\mathrm{s}_{\max }$

. $\mathrm{s}_{\min }$

$\mathrm{S}_{\mathrm{op}}$

$\triangle \mathrm{S}$

$\triangle \mathrm{s}_{\text {eff }}$

$\mathrm{U}$

half crack length

initial half crack length

empirical constant

specimen thickness

empirical constant

Young's Modulus

stress intensity factor

empirical constant

applied load

effective stress range

distance between the center of the crack and the contact line

location of crack splitting force with respect to specimen centerline

the magnitude and distribution of closure force

the critical stress intensity for failure or fracture toughness

the maximum stress intensity factor in a loading cycle

the mean value of stress intensity factor in a loading cycle

the minimum stress intensity factor in a loading cycle

the range of stress intensity factor in a loading cycle; $\Delta \mathrm{K}=\mathrm{K}_{\max }-\mathrm{K}_{\min }$

the effective range of stress intensity factor in a loading cycle

the maximum applied load in a loading cycle

the minimum applied load in a loading cycle

the applied load above which the crack is fully open

stress ratio; $\mathrm{R}=\mathrm{S}_{\min } / \mathrm{S}_{\max }=\mathrm{K}_{\min } / \mathrm{K}_{\max }$

applied (gross-section) stress; $\mathrm{S}=\mathrm{P} / \mathrm{BW}$

the maximum applied stress in a loading cycle

the minimum applied stress in a loading cycle

crack opening stress above which the crack tip is fully open

the range of applied stress in a loading cycle; $S=S_{\max }-S_{\min }$

effective stress or stress intensity range ratio; $U=\frac{\Delta S_{\text {eff }}}{\Delta S}=\frac{\Delta K_{\text {eff }}}{\Delta K}$ 
v y direction displacement

$\mathrm{v}_{\text {closure }} \mathrm{y}$ direction displacement produced by the closure force

$\mathrm{v}_{\text {remote }} \mathrm{y}$ direction displacement produced by remote loading

$\mathrm{v}_{\text {splitting }} \mathrm{y}$ direction displacement produced by two pairs of unit splitting force applied to the crack faces

$\mathrm{V} \quad$ electrical potential between the potential leads

$\mathrm{V}$ (a) electrical potential between the potential leads

$\mathrm{V}\left(\mathrm{a}_{\mathrm{o}}\right) \quad$ electrical potential between the potential leads corresponding to half-crack length $a_{0}$

W gross specimen width

$\overline{\bar{Z}}(\mathrm{z}), \overline{\mathrm{Z}}(\mathrm{z}), \mathrm{Z}(\mathrm{z}), \mathrm{Z}^{\prime}(\mathrm{z}) \quad$ stress function and its derivatives

$\epsilon_{\mathrm{y}} \quad$ longitudinal strain

$(\epsilon$ y) closure longitudinal strain produced by closure force

$(\epsilon$ ) remote longitudinal strain produced by remote loading

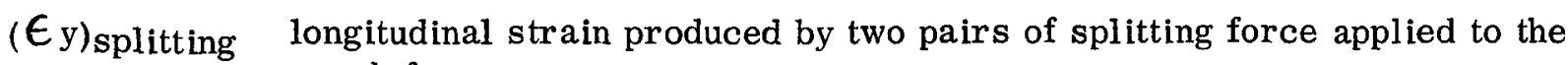
crack faces

$\downarrow \quad$ Poisson ratio

$\sigma \quad$ applied stress

$\sigma \times \quad$ longitudinal stress

$\sigma \mathrm{y}$ transverse stress

$\sigma$ ys yield strength

$\tau \mathrm{xy} \quad$ shear stress

$\Phi(\mathrm{x}, \mathrm{y}) \quad$ Airy's stress function

$\Psi_{\mathrm{i}} \quad$ harmonic functions; $i=1,2,3,4$ 


\section{INTRODUCTION}

The importance of fatigue in determining the serviceable life of engineering structures has been well recognized [1]. In modern high-performance structure designed for finite service life, fatigue crack growth occurs over a significant portion of the useful life of the structure. Information on the kinetics of fatigue crack growth, therefore, becomes an important aspect of material selection and design.

It has been shown that the crack tip stress intensity factor, $K$, defined by linear elasticity, is the most appropriate parameter for characterizing crack growth $[2,3,4]$. Several empirical relationships for correlating fatigue crack growth data have been suggested [2, 5-8]. Paris et al [7] and Paris and Erdogan [8] suggested that the primary variable of importance for fatigue crack growth is the range of the crack tip stress intensity factor, $\Delta K$, and that the rate of fatigue crack growth, $\Delta \mathrm{a} / \Delta \mathrm{N}$, may be described by a relationship given by Equation 1.

$$
\frac{\Delta \mathrm{a}}{\Delta \mathrm{N}}=\mathrm{A}(\Delta \mathrm{K})^{\mathrm{n}}
$$

$A$ and $n$ are empirical constants. The mean value, or the maximum value of $K$ $\left(\mathrm{K}_{\text {mean }}\right.$ or $\left.\mathrm{K}_{\max }\right)$ in a given load cycle was thought to have only a secondary - effect on crack growth. Forman et al [6] recognized that the maximum value of $K, K_{\max }$, can have a significant effect on fatigue crack growth, particularly at maximum $\mathrm{K}$ levels approaching $\mathrm{K}_{\mathrm{c}}$ (the critical stress intensity for failure). They suggested that Equation 1 be modified so that the rate of fatigue crack 
growth would approach infinity as $\mathrm{K}_{\max }$ approaches $\mathrm{K}_{\mathrm{c}}$, Equation 2.

$$
\frac{\Delta \mathrm{a}}{\Delta \mathrm{N}}=\frac{\mathrm{A}(\Delta \mathrm{K})^{\mathrm{n}}}{(1-\mathrm{R}) \mathrm{K}_{\mathrm{c}}-\Delta \mathrm{K}}
$$

The stress ratio, $R$, is the ratio between the minimum and maximum $K$ levels in a load cycle; $R=\mathrm{K}_{\min } / \mathrm{K}_{\max }$. Reasonable correlation with fatigue crack growth data in the range of $10^{-6}$ to $10^{-3}$ inch per cycle $\left(2.54 \times 10^{-5}\right.$ to $2.54 \mathrm{x}$ $10^{-2} \mathrm{~mm}$ per cycle) has been demonstrated [9].

The implicit assumptions in both Equations 1 and 2 are (a) that only the tensile portion of the load cycle is effective in producing fatigue crack growth, and (b) that there is no crack closure over the tensile portion of the load cycle, so that the full tensile range of loading is effective. In a recent series of experiments on a 2024-T3 aluminum alloy, Elber observed that the load versus crack-opening-displacement curves exhibited a nonlinear region at the lower load levels, as indicated schematically in Figure 1 [10]. This behavior was interpreted in terms of crack closure, that is, physical contact between the fracture surfaces produced by fatigue. Elber suggested that the crack is closed at the tip over the lower porition of the loading cycles and becomes open only after the applied stress exceeded a level $\mathrm{S}_{\mathrm{op}}$ (Figure 1) and that fatigue crack growth can occur only during that portion of the loading cycle in . which the crack is fully open. Based on this suggestion, an effective stress range, $\Delta S_{\text {eff }}$, and an effective stress range ratio, $U$, were defined.

$$
U=\frac{\Delta S_{\text {eff }}}{\Delta S}=\frac{S_{\max }-S_{o p}}{S_{\max }-S_{\min }}
$$

$\mathrm{S}_{\max }$ and $\mathrm{S}_{\mathrm{min}}$ are the maximum and minimum values of the applied stress in 
a given cycle; and $\mathrm{S}_{\mathrm{op}}$ is the crack opening stress. U can be defined equivalently in terms of the effective stress intensity range, $\Delta K_{\text {eff }}$, and $\Delta K$.

$$
\mathrm{U}=\frac{\Delta \mathrm{K}_{\mathrm{eff}}}{\Delta \mathrm{K}}=\frac{\mathrm{K}_{\max }-\mathrm{K}_{\mathrm{op}}}{\mathrm{K}_{\max }-\mathrm{K}_{\min }}
$$

$\mathrm{K}_{\mathrm{op}}$ is the crack opening stress intensity factor corresponding to $\mathrm{S}_{\mathrm{op}}$. Elber further suggested that the crack growth relationship be written in the following form:

$$
\frac{\Delta \mathrm{a}}{\Delta \mathrm{N}}=\mathrm{C}\left(\Delta \mathrm{K}_{\mathrm{eff}}\right)^{\mathrm{n}}=\mathrm{C}(\mathrm{U} \triangle \mathrm{K})^{\mathrm{n}}
$$

Based on a limited range of data, Elber suggested that the effective stress range ratio, $U$, is a function of the stress ratio, $R$, and is independent of $\mathrm{S}_{\max }$, or $\mathrm{K}_{\max }$. For the 2024-T3 aluminum alloy sheet, $\mathrm{U}$ is given simply by Equation 6, for $\mathrm{R}$ value ranging from -0.1 to 0.7 .

$$
\mathrm{U}=0.5+0.4 \mathrm{R} \quad-0.1 \leqslant \mathrm{R} \leqslant 0.7
$$

Using this empirical result, Elber showed that Equation 5 provided a better fit to the experimental data than either Equation 1 or Equation 2 [10]. Because Equation 6 was based on a very limited amount of data and because much of the data was obtained under conditions of net-section yielding, this relationship for $U$ should be considered only as a preliminary estimate and must be verified by additional experimentation. Thus far, no independent verification of this result has been reported, although some direct evidence for closure has been given by Buck et al [11-13]. Nevertheless, Elber's concept of effective stress range has been adopted by several authors $[14,15]$, and the expression for $U$ (Equation 6) has been accepted and used without question by others [16-18]. 
The principal purpose of this work is: (a) to provide independent verification of the crackclosure phenomenon; (b) to determine the effects of stress ratio, $R$, and maximum stress intensity factor, $K_{\max }$, on closure; and (c) to develop an analytical model for estimating the magnitude and distribution of the closure forces. The experimental and analytical studies are carried out within the framework of linear-elastic fracture mechanics. A Ti-6Al-4V alloy sheet is used in the experimental studies on closure. Crack closure is measured directly by means of an electrical potential technique, and indirectly by measurements of strains in the neighborhood of the crack tip. The implications of this study to the understanding of several practical problems in fatigue crack growth are considered.

Acknowledgment is extended to Mr. J. H. FitzGerald for his invaluable contributions to the experimental work. 


\section{MATERIAL AND EXPERIMENTAL WORK}

\section{A. Material and Specimen}

A 0.2 -inch-thick $(5.08 \mathrm{~mm})$ mill annealed $\mathrm{Ti}-6 \mathrm{Al}-4 \mathrm{~V}$ alloy plate was used in this investigation. The chemical composition, and longitudinal and transverse tensile properties of this alloy are given in Table I.

Three-inch-wide $(76.2 \mathrm{~mm})$ by 16 -inch-long $(406 \mathrm{~mm})$ center-cracked specimens (Figure 2), oriented in the long transverse (TL) direction, were used in the fatigue crack growth and crack closure studies. The initial center notch, about 0.4 inch (about $10 \mathrm{~mm}$ ) long, was introduced by electro-discharge machining (EDM). The specimens were precracked in fatigue either at loa ds to be used in the subsequent studies, or through a decreasing sequence of loads that terminated at these loads. The precracking procedure provided fatigue cracks about 0.08 inch (about $2 \mathrm{~mm}$ ) in length from the ends of the starter notches corresponding to a half-crack length of about 0.3 inch $(7.6 \mathrm{~mm})$, and ensured that the subsequent tests will be unaffected by the starter notch geometry and by the residual stress produced by EDM. Both precracking, and the crack growth and. crack closure experiments were carried out in a 100,000-lb. capacity MTS system closed-loop electrohydraulic testing machine. Load control was estimated to be better than \pm 1 percent.

The stress intensity factor, $\mathrm{K}$, for the center-cracked specimen was computed from Equation 7:

$$
\mathrm{K}=\frac{\mathrm{P}}{\mathrm{BW}} \sqrt{\pi \mathrm{a} \sec (\pi \mathrm{a} / \mathrm{W})}
$$

where $\mathrm{P}=$ applied load, $\mathrm{B}=$ specimen thickness, $\mathrm{W}=$ gross specimen width, and $\mathrm{a}=$ half crack length. The secant term is a correction for finite specimen 
width [19] which closely approximates the series correction obtained by Isida $[20]$.

\section{B. Crack Monitoring System}

An electrical potential method was used for monitoring crack growth and closure utilizing a continuous recording system. This method is based on an increase in electrical resistance of the specimen with crack growth. A constant current is applied to the specimen, and changes in electrical potential are measured between fixed points above and below the crack, Figure 3. A schematic diagram of the system is shown in Figure 4. The relationship between the electrical potential, $V$, half crack length, $a$, and the distance between the two fixed measurement points, $2 \mathrm{y}$, for the central cracked specimen is given by Equation 8:

$$
\frac{V(a)}{V\left(a_{0}\right)}=\frac{\cosh ^{-1}\left(\frac{\cosh \pi y / W}{\cos \pi a / W}\right)}{\cosh ^{-1}\left(\frac{\cosh \pi y / W}{\cos \pi a_{0} / W}\right)}
$$

This equation, derived by Johnson [21] was used as an analytical calibration curve. The electrical potential method provides measurements of average crack length through the thickness, as opposed to the visual methods which give measurements of the crack length at the specimen surface only. This method has been shown to agree well with other crack measuring techniques for a number of materials tested in various environments, provided that crack tunneling is taken into account [22].

This method of crack measurement has several advantages. It permits 
measurements of crack length wh ile the crack is completely covered, thus giving complete freedom for using environmental chambers which may completely cover the crack area. Since changes in electrical potential reflect electrical shorting, vis-a-vis, physical contact, across the crack surfaces, these changes provide direct measures of crack closure. The other important advantages are that it gives a continuous measurement of crack length as a function of time and that it permits continuous recording of load-potential curves. By using a working current of about 2.1 amperes, this system provided an average measurement sensitivity of about 0.0025 inch $(0.064 \mathrm{~mm})$ in half crack length, a, per microvolt (uv) changes in potential (that is, $0.0025 \mathrm{inch} / \mu \mathrm{v}$, or $0.064 \mathrm{~mm}$ $\mu v)$ for these specimens. Resolution is better than 0.001 inch, or $0.025 \mathrm{~mm}$. For autographic recording of the load versus potential data used in the crack closure studies, an alternate amplification procedure was used in place of the system shown in Figure 4. The electrical potential signal from the specimen was applied directly to the input of a d-c amplifier in the MTS Systems testing machine. The output from this amplifier was reduced by a preset $d-c$ signal, and the difference signal was amplified further in the $x-y$ recorder and recorded. Because these amplifiers were designed for signal levels in the millivolt range, and the changes in electrical potential were in the microvolt range, the background noise tended to be higher with this method. The reduced signal to noise ratio, however, did not seriously affect the closure results (see Figure 6, for example) and was acceptable in view of the improved convenience in measurement. 


\section{Environment Control System}

Preliminary experiments indicated that an insulating oxide layer formed on the fracture surfaces of specimens tested in air. This insulating oxide layer interferred with the measurement of crack closure by the electrical potential method, and gave values that underestimated the extent of closure. To circumvent this problem, all electrical potential measurements of closure were carried out on specimens tested in dehumidified argon that had been further purified by a titanium sublimation pump. A schematic diagram of the environment control system used in these experiments is shown in Figure 5.

Purification was achieved by passing ultrahigh purity argon through a molecular sieve drier, and a series of cold traps at about $-140^{\circ} \mathrm{C}$. Further purification was obtained by passing the argon through a titanium sublimation pump (TSP) in line with the gas purification system. The TSP was operated as a getter, and was connected directly to the environment chamber through a high conductance tube. To reduce possible back diffusion of impurities, the argon was discharged through an additional cold trap and a silicone fluid trap. The effectiveness of this purification system has been demonstrated by Wei and Ritter [23].

\section{Experimental Work}

Two independent methods were used to study the crack closure phenomenon. Crack closure was measured directly by using the electrical potential method. Indirect measurements of crack closure were made by measuring strains in regions near the crack tip. To simplfy experimentation, only one of 
the methods was used on each test specimen. The effects of both $R$ and $K_{\max }$ were examined.

Closure experiments were carried out, at suitable crack length intervals, on specimens that have been fatigued under constant load-amplitude cycling at selected stress ratios, $R$. Stress ratios of $0.05,0.1,0.2,0.3$ and 0.5 were used with the electrical potential method. For the strain measurements, only $R$ of 0.05 was utilized. A constant maximum load, $P_{\max }$, of $12,000 \mathrm{lb}$ (53.38 kN) was used for all the tests except for those at $R=0.05$. For the tests at $R=0.05, P_{\max }$ of $10,000 \mathrm{lb} .(44.48 \mathrm{kN})$ was used. Fatigue cracks were extended from an initial length of about 0.3 inch to about 0.9 inch (or, from about 7.6 to $23 \mathrm{~mm}$ ). The corresponding values of $\mathrm{K}_{\max }$ ranged from about 15 to $33 \mathrm{ksi}-\mathrm{in}^{\frac{1}{2}}\left(16.5\right.$ to $\left.36.3 \mathrm{MN}-\mathrm{m}^{-3 / 2}\right)$ for the test at a $\mathrm{P}_{\max }$ of $10,000 \mathrm{lb}$. (44.48 kN), and from about 17 to $40 \mathrm{ksi}-\mathrm{in}^{\frac{1}{2}}\left(18.7\right.$ to $\left.44 \mathrm{MN}-\mathrm{m}^{-3 / 2}\right)$ for tests at $\mathrm{P}_{\max }$ of $12,000 \mathrm{lb} .(53.38 \mathrm{kN})$.

1. Electrical Potential Measurements

Electrical potential measurements of crack closure were carried out on specimens that were fatigued in dehumidified argon (see section on Environment Control System). Fatigue cracks were extended to various selected lengths under constant load-amplitude cycling at $5 \mathrm{~Hz}$. for prescribed $\mathbf{R}$ and $\mathrm{P}_{\max } \cdot$ Cyclic loading was interrupted at selected crack length intervals, with the load at $\mathrm{P}_{\max }$, and the closure experiments were performed. The following sequences were utilized and constitute one set of closure experiments:

(a) the specimen was unloaded from $P_{\max }$ to $P_{\min }$, and then reloaded to $P_{\max }$; 
(b) the specimen was then unloaded from $P_{\max }$ into compression to about 4,000 lb. (17.79 $\mathrm{kN}$ compression), and reloaded to $\mathrm{P}_{\max }$; and (c) finally, step (a) was repeated. These procedures were carried out using the one-cycle loading feature on the MTS machine operated at $0.01 \mathrm{~Hz}$. Autographic recordings of load versus change in electrical potential (vis-a-vis, change in apparent crack length - crack closure) were made during each of the aforementioned steps.

\section{Strain Measurements}

For convenience, specimens for the strain measurements were fatigued in air. Metal foil strain gages, with $0.015 \mathrm{inch}(0.38 \mathrm{~mm})$ gage length, were used. Up to 4 strain gages were mounted at various locations on the specimen surface adjacent to the line of intended crack prolongation (see Table 2 for specific locations for the strain gages). Fatigue cracks were extended under constant amplitude cyclic loading at $5 \mathrm{~Hz}_{\bullet}$, with $\mathrm{P}_{\max }=$ $10,000 \mathrm{lb} .(44.48 \mathrm{kN})$ and $\mathrm{R}=0.05$. Cyclic loading was again interrupted at selected intervals (to approximate those of the electrical potential measurements) for the closure studies. Here, only the load sequence $P_{\max }$ to $4,000 \mathrm{lb}$. compression (-17.79 kN) back to $\mathrm{P}_{\max }$ was used. Autographic recordings of load versus strain were made during these unloading and reloading cycles. 


\section{RESULTS AND DISCUSSIONS}

In this section, the experimental results will be summarized. The results of the electrical potential and strain measurements will be considered separately. The effects of stress ratio, $R$, and maximum stress intensity factor, $\mathrm{K}_{\mathrm{max}}$, on crack closure will be discussed. Detailed interpretations of these experimental results and correlations with an approximate analysis will be given in the following section on analytical modeling.

\section{A. Electrical Potential Measurements}

Typical load versus change in electrical potential curves are shown in Figure 6-8 for the sets of crack closure experiments described in paragraph D-1 of Section II. Figure 6 depicts those for loading sequence (a) -- $P_{\max }$ to $P_{\min }$ to $P_{\max } ;$ Figure 7 , those for sequence (b) $--P_{\max }$ to $-4000 \mathrm{lb} .(-17.79 \mathrm{kN})$ to $P_{\max }$; and Figure 8, those for sequence (c) -- $P_{\max }$ to $P_{\min }$ to $P_{\max }--$ following the sequence into compression. Each of the curves corresponds to a different crack length, and thus corresponds to a different value of $\mathrm{K}_{\max }$. Sensitivity ranged from about $0.005 \mathrm{in} / \mu \mathrm{v} .(0.13 \mathrm{~mm} / \mu \mathrm{v}$.$) to 0.002 \mathrm{in} / \mu \mathrm{v}$ $(0.05 \mathrm{~mm} / \mu \mathrm{v})$, corresponding to crack lengths, a, of 0.3 to $0.8 \mathrm{in}$., respectively.

Changes in electrical potential reflects electrical (and, hence physical) contact between the fracture surfaces, and thus provide a direct measure of crack closure. For a crack in an elastic medium, the crack surfaces are expected to be completely separated (open) under an externally applied tensile load, and to be in complete contact (fully closed) in compression. Load versus change in electrical 
potential curves for this idealized case are expected to follow the behavior indicated by Figure 9. In the tensile region, the electrical potential assumes some value $V($ a) corresponding to the prevailing crack length. As the applied load is reduced through zero into compression, the electrical potential undergoes a step-wise change from $\mathrm{V}(\mathrm{a})$, and assumes a value $\mathrm{V}\left(\mathrm{a}_{\mathrm{o}}\right)$, corresponding to that for some initial finite-width notch of length $a_{0}$, or a value corresponding to the uncracked specimen. In reality, if crack closure occurs, it is expected to proceed from the crack tip and extend gradually back towards the initial notch. This gradual change is reflected in the actual experimental data, Figures 6-8. Initial deviation from $\mathrm{V}(\mathrm{a})$ can be identified with the onset of crack closure. This point can be identified with the crack opening stress, $S_{\mathrm{op}}$, used by Elber [10], and will be utilized in computing U. (Note that, aside from the identification with the onset of crack closure, no further physical significance is assumed or implied. Further discussion of this point will be given in a later section.) The influences of $R$ and $K_{\max }$ on the onset of crack closure will be considered separately in a later section.

It is useful to consider the nature of the load versus change in electrical potential curves in some detail, which serves to qualify the experimental results and provides some physical insight into the crack closure phenomenon. It is important to recognize that the electrical potential measurements provide measures of the average (through the thickness average) crack lengths, and 
that the identification of a change in electr ical potential with an increment of crack closure is based on the assumption that the electrical conductance across the fracture faces approximates that of the undamaged material. It is believed that this assumption was reasonably well met, and was correct in the case of compression loading based on the agreement between the electrical potential under compression loading and that of the uncracked specimen. Irrespective of this assumption, the point for the onset of crack closure can be identified with good accuracy.

From the experimental data, it can be seen that the paths of the unloading and reloading curves were somewhat different. The differences are particularly apparent in those cases where the specimens were loaded into compression. The differences were caused in part by short-term drift and electrical noise in the measurement circuit. The principal difference, Figure 7, was produced, most likely by crushing of the fracture surfaces, and by refracturing of regions of the fracture surfaces that had become "cold welded" during compression.

\section{B. Strain Measurements}

For an idealized crack in an elastic medium, as considered in the previous section, the load versus strain curves will exhibit two types of behavior depending on whether the strain gage is located ahead of, or behind the crack tip. For a gage that is located ahead of the crack tip, the idealized behavior would be that shown in Figure 10(a). In the tension region, the behavior is linear and reflects the stress (strain) concentration effect of the crack. In compression, the slope of the load-strain curve corresponds to that of an 
uncracked specimen. The abrupt change in slope at zero load reflects the abrupt change in stiffness as this idealized crack changes from a fully open to a fully closed configuration. Figure 10(b) illustrates the idealized load-strain behavior, when the strain gage is located behind the crack tip. In the compression region, the load strain curve again corresponds to that of an uncracked specimen. In the tension region, since the strain (stress) is essentially unloaded by the presence of the crack, changes in strain with load become very small. Similar to the case represented by Figure 10(a), the abrupt change in slope at zero load is again that associated with opening and closing of the crack.

The load versus strain behavior for cracks in real material is represented by the curves shown in Figures 11. The load was aga in cycled from $\mathrm{P}_{\max }$ into compression, and then back to $\mathrm{P}_{\max }$. The curves represent measurements made on two separate gages, and reflect changes in load-strain behavior as the crack tip approached and then moved away from the gage. The substantial deviations in behavior from those of the idealized crack again reflects the gradual nature of the closure process. The onset of closure can be detected most easily when the crack tip is close to the strain gage, and is determined by the deviation from linear behavior in the tension region. The loads at which the onset of closure began correlate well with the loads obtained from the electrical potential measurements. A more detailed discussion of these results is best made in terms of an analytical model, and will be deferred. 
C. The Effects of Stress Ratio and $\mathrm{K}_{\max }$ on Crack Closure

On the bas is of the electrical potential data, Figures 6 and 7 , the effect of stress ratio, $R$, and the maximum stress intensity, $K_{\max }$, on crack closure may be considered. Crack closure is considered to begin when the electrical potential deviates from the value at full load. The point for the onset of crack closure is consistent with that defined by Elber [10] on the basis of crack opening displacement measurements. The results are shown in Figure 12 , in terms of $U$ (the effective stress range ratio, U, as defined by Elber). It is to be emphasized again that $U$ is to be interpreted here only in terms of the onset of crack closure. No further physical significance is assumed or implied.

$U$ and $P_{o p}$, corresponding to the onset of crack closure, are shown in Figures $12 \mathrm{a}$ and $12 \mathrm{~b}$ as functions of $\mathrm{K}_{\max }$ for various $\mathrm{R}$. These results clearly indicate that the onset of closure is a function of stress ratio, as well as a function of $\mathrm{K}_{\max }$ and hence of crack length. For $\mathrm{R}$ greater than 0.3 , no crack closure was observed (i.e. $P_{o p} \leq P_{\min }$ ), and $U=1$. For values of $R$ below 0.3 , $U$ is a function of $K_{\max }$ above certain $K_{\max }$ values, and is independent of $K_{\max }$ (i.e. $U$ approaches 1) below these $K_{\max }$ values, Figure 12a. It is dependent on $K_{\max }$ in an intermediate range, and becomes mildly dependent on $K_{\max }$ at high $\mathrm{K}_{\max }$ levels. The latter region corresponds to the range in which $\mathrm{P}_{\text {op }}$ becomes nearly constant, Figure $12 \mathrm{~b}$. These results are qualitatively consistent with the physical model (see next section) that suggested an influence of stress ratio, $R$, and are consistent with the probable dominance of the surface plastic zones on closure. 
The results are, however, in contrast with those reported by Elber [10] for a 2024-T3 aluminum alloy. The differences, of course, can be attributed in part to the differences in materials. The principal causes, however, reside in Elber's data. First, because of the limited range of data (obtained mostly at high $\mathrm{K}_{\max }$ levels) and of the inherent uncertainties in the results, Elber's assertion that $U$ is independent of $K_{\max }$ cannot be regarded as being conclusive. Second, most of Elber's data were obtained under conditions above net section yielding. As such, the utility of the data and of the suggested relationship between $U$ and $R$ is questionable. Further independent verifications are needed.

\section{Discussion}

Based on the experimental observations, the following processes for crack closure are envisioned. The physical basis for closure can best be described by comparison with idealized cases. For an idealized stationary crack (infinitesimally thin slit) in an elastic medium, the crack surfaces are expected to be completely separated (open) under an externally applied tensile load. The separation between the crack faces (crack opening displacement), can be defined by linear elastic analysis [24]. Upon unloading to zero load, the crack opening displacement would return to zero along the full length of the crack. For an idealized stationary crack in an elastic-plastic medium, the crack opening displacements (crack contour) under tension are expected to be larger than those of the elastic case at the same load, as a result of plastic 
deformation ahead of the crack tip [25]. Because of this plastic deformation, residual crack opening displacements will remain following the reduction to zero load, and no crack closure would be indicated.

Real cracks, however, are formed as a result of highly localized deformation and separation at the crack tip, and are expected to behave quite differently. It is envisioned that the process of deformation and separation (for example, that suggested by Krafft $[26,27])$ followed by compaction, would produce a highly deformed layer. This layer, defined as "a layer of residual tensile strain left in the wake of the crack tip, " [10] delineates an effective crack contour with crack opening displacements that are, in all probability, smaller than those of an equivalent elastic crack. On unloading, the surfaces of this layer may come into contact and give rise to the observed results. Of course, the degree of compaction depends on the ratio between the minimum and maximum loads in a cycle, that is, on stress ratio, $R$. Hence the onset of crack closure is expected to be a function of R. Since the extent of the residual deformation is dependent on $\mathrm{K}_{\max }$, the onset of crack closure is expected to be a function of $K_{\max }$, also. The observations of experimental results support this model. 


\section{MODELING FOR CRACK CLOSURE}

A two-dimensional elastic model is used to assist in the interpretation of experimental results. It is recognized that such a model cannot truly represent the real physical problem, which is one that involves elastic-plastic behavior, with unloading, and is most probably three-dimensional in nature [28]. Nevertheless, it is felt that some semi-quantitative understanding can be obtained. In the following sections a brief description of the model is given, and the qualitative features of the model are discussed in terms of the observed load-strain behavior. On the basis of the model and the experimental data, estimates of the crack closure forces and the load versus crack opening displacement curves are made and discussed.

\section{A. Analytical Model}

The model chosen is that of a notch-like crack in a homogeneous, isotropic elastic body loaded by remote tension and by distr ibuted compressive forces, representing forces produced by crack closure, on the crack faces as shown in Figure 13. The magnitude and distribution of the distributed forces are expected to depend on the degree of closure. It is assumed that the crack faces remain separated, and the only manifestation of closure is the artifical introduction of closure forces. It is further assumed that the closure forces are uniformly distributed through the thickness (that is, in the z-direction), and vary only in the $\mathrm{x}$-direction, or along the crack length direction. The problem is then treated as one of generalized plane stress. Based on these assumptions, the 
stresses (strains) at any point can be obtained by superposition, Figure 13. This model is analogous to the strip yield model proposed by Dugdale [29], and is expected to provide reasonable approximate solutions for stresses (strains) away from the crack tip. The approximations are expected to be poor, however, in the near-tip region.

For a plate containing a central through-thickness crack of length $2 a$, the strain at any point and the relative displacement between any two points in the plate may be determined by using the Westergaard method and the appropriate stress functions [24]. The Westergaard method and the derivation of the relevant strain and displacement equations are summarized in the Appendix. The longitudinal strain, $\epsilon_{y}$, and displacement, v, produced by ${ }^{\prime}$ remote loading on a finite width plate are given by Equations 9 and 10:

$$
\begin{aligned}
& (\mathrm{Ev})_{\text {remote }}=\frac{\mathrm{P}}{\mathrm{BW}}\left[2 \operatorname{Im}\left(\sqrt{\mathrm{z}^{2}-\mathrm{a}^{2}}\right)-(1+\nu) \text { y } \operatorname{Re}\left(\frac{\mathrm{z}}{\sqrt{\mathrm{z}^{2}-\mathrm{a}^{2}}}\right)\right] \sqrt{\sec \frac{\pi \mathrm{a}}{W}} \\
& \left(\mathrm{E} \epsilon_{\mathrm{y}}\right)_{\text {remote }}=\frac{\mathrm{P}}{\mathrm{BW}}\left[(1-\nu) \operatorname{Re}\left(\frac{\mathrm{z}}{\sqrt{\mathrm{z}^{2}-\mathrm{a}^{2}}}\right)+(1+\nu) \mathrm{y} \operatorname{Im}\left(\frac{-\mathrm{a}^{2}}{\sqrt{\left(\mathrm{z}^{2}-\mathrm{a}^{2}\right)^{3}}}\right)\right] \sqrt{\sec \frac{\pi \mathrm{a}}{W}}
\end{aligned}
$$

For the present discussions, these equations are assumed to be valid also for compression loading, since the crack is assumed to be notch-like and to remain separated. The longitudinal strain and displacement produced by the distributed closure forces are obtained by integration of the solutions for two pairs of unit splitting forces applied to the crack faces, Figure 14. The longitudinal strain and displacement by the unit splitting forces are given by Equations 11 and 12: 


$$
\begin{aligned}
(E v)_{\text {splitting }}= & \frac{2}{\pi}\left[2 \operatorname{Im}\left(\tan \sqrt[-1]{\frac{z^{2}-a^{2}}{a^{2}-b^{2}}}\right)-(1+\nu) \text { ye }\left(\frac{z \sqrt{a^{2}-b^{2}}}{\left(z^{2}-b^{2}\right) \sqrt{z^{2}-a^{2}}}\right)\right] x \\
& \sqrt{\sec \frac{\pi a}{W}} \\
\left(E \epsilon_{y}\right)_{\text {splitting }}= & \frac{2}{\pi}\left[(1-\nu) \operatorname{Re}\left(\frac{z \sqrt{a^{2}-b^{2}}}{\left(z^{2}-b^{2}\right) \sqrt{z^{2}-a^{2}}}\right)-(1+\nu) y \operatorname{Im}\left(\frac{a^{2} \sqrt{a^{2}-b^{2}}}{\left(z^{2}-b^{2}\right) \sqrt{\left(z^{2}-a^{2}\right)^{3}}}\right.\right. \\
& \left.\left.+\frac{2 z^{2} \sqrt{a^{2}-b^{2}}}{\left(z^{2}-b^{2}\right)^{2} \sqrt{z^{2}-a^{2}}}\right)\right] \sqrt{\sec \frac{\pi a}{W}}
\end{aligned}
$$

The longitudina! strain and displacement produced by the distributed crack closure forces are then given by Equation 13 and 14:

$$
\begin{aligned}
& (E v)_{\text {closure }}=\int_{\text {close }}^{a}(E v)_{\text {splitting }} f(b) d b \\
& \left(E \epsilon_{y}\right)_{\text {closure }}=\int_{a_{\text {close }}}^{a}\left(E \epsilon_{y}\right)_{\text {splitting }} f(b) d b
\end{aligned}
$$

The longitudinal strain and displacement produced by the combination of remote loading and crack closure forces are then obtained by the superposition of the solutions given by Equation 15 and 16:

$$
\begin{aligned}
& E v=(E v)_{\text {remote }}+(E v)_{\text {closure }} \\
& E \epsilon_{y}=\left(E \epsilon_{y}\right)_{\text {remote }}+\left(E \epsilon_{y}\right)_{\text {closure }}
\end{aligned}
$$

The following definitions of terms were used for the previous equations:

$$
\begin{aligned}
& \mathrm{z}=\mathrm{x}+\mathrm{i} \mathrm{y} ; \mathrm{x}, \mathrm{y}, \text { coordinate of the point } \\
& \mathrm{E}, \mathcal{V}=\text { Young's modul us and Poisson ratio respectively }
\end{aligned}
$$




$$
\begin{gathered}
\mathrm{B}, \mathrm{W}=\text { thickness and width of the specimen, respectively } \\
\mathrm{b}=\text { the distance between the center of the crack and splitting force } \\
\mathrm{a}_{\text {close }}= \\
\text { the distance between the center of the crack and the contact } \\
\mathrm{f}(\mathrm{b})=\text { the magnitude and distribution of closure force } \\
\sqrt{\sec (\pi a / W)}=\text { correction factor for the finite width specimen }
\end{gathered}
$$

Unfortunately, the magnitude and distribution of the crack closure forces are not known. Under the assumptions of the model, an estimate may be made on the basis of the crack closure data and the load-strain results. Before proceeding with this estimate, qualitative features of this model may be examined and discussed in terms of the load-strain results.

\section{B. Behavior of Load-Strain Curves}

To facilitate the discussion of the load-strain curves, the longitudinal strains at fixed gage locations produced by unit splitting forces were computed as a function of the location of these forces, and are shown in Figures 15 and 16. Two different crack lengths were chosen. For one, all of the gages were located ahead of the crack tip; and the other, all behind the crack tip. The crack tips were sufficiently far away from the gages in both cases such that the approximations would be reasonable. Some specific cases are discussed below.

1. Strain Gage Located Ahead of the Crack Tip

A typical load-strain record for a strain gage located ahead of the crack tip is shown in Figure 17. This curve deviated from the load-strain 
curve for a notch-like elastic crack whenever the crack closure occurred. Whenever there is crack closure, compressive forces acting on the crack sur-. faces are expected. These closure forces produce tensile strain at the point ahead of the crack tip, just like a wedge force acting on the crack (Figure 15). The tensile strain produced by the closure forces makes the load-strain curves deviate from the idealized one. Upon further unloading, both the closed area and the closure force increase, so that the strain-load curve deviates further away from the idealized curve. The shadowed area depicted in Figure 17 represents the effect of the closure force.

\section{Strain Gage Located Just Behind the Crack Tip}

A typical load-strain curve for a strain gage located just behind the crack tip is shown in Figure 18. Initial deviation from the curve for the notchlike elastic crack before the onset of crack closure may be attributed to the onset of reversed yielding [30]. Once closure occurred, the curve deviated substantially from the idealized one. Now, the closure forces work like distributed forces acting on a straight boundary, and produce compressive strain on the gage points. The shadowed area in Figure 18 depicts the influence of the closure force and the cross hatched area shows approximately the influence of reversed yielding. Since the strain caused by closure forces and by reversed yielding are compressive instead of tensile, the shadowed area in Figure 18 is on the other side of the idealized curve when compared with the shadowed area in Figure 17.

It should be noted that the rate of decrease of strain was very high just 
after the onset of crack closure, and slowed down and approached a constant with further unloading. The initial rapid decrease was caused by both the rapid increase in closed area and of the closure force effect. As the contact line moved further away from the strain gage, the closure force on the newly closed area produced little additional strain on the strain gage. Hence, the rate of decrease became principally a function of the remotely applied load.

\section{Strain Gage Located Far Behind the Crack Tip}

For constant amplitude loading, the electrical potential measurements indicated that the onset of crack closure occurred at increasingly higher loads with increasing $K_{\max }$, that is, with increasing crack lengths, Figure $12 \mathrm{~b}$. Strain gage measurements (Figure 11b) appear to suggest, however, that the onset of crack closure occurred at progressively lower loads for the longer crack lengths. This apparent discrepancy was caused by the reduction in sensitivity when the strain gage is far away from the crack tip and can be explained in terms of the influence line depicted in Figure 16. Although the onset of crack closure actually occurred at a higher load for a longer crack length (as indicated by the electrical potential method), the strain produced at the gage location (when the crack tip was well past the gage) was small because of its remoteness from the closure forces near the crack tip. (Of course, the strain produced at the gage location by reversed yielding at the crack tip would have been small also). The strain gage could sense the effect of crack closure only when the contact line approached the gage. This occurred at loads much lower than that at the onset of closure, as shown in Figure 11b. Examination of Figure 11 shows that the strain produced by the splitting force changed signs depending on the 
location of the force in relation to the strain gage; being tensile over certain regions far removed from the strain gage and compressive near the gage. This change in sign, in combination with changes in magnitude with location, is consistant with the observed behavior.

\section{Estimate of Closure Forces}

An estimate of the magnitude and distribution of the closure force may be made on the basis of the superposition model, and experimental data on closure and on load-strain variations. The estimate was made for the case of $R=0.05$, since electrical potential measurements of crack closure and companion loadstrain data were available. The extent of crack closure (through-thickness average) was determined from the electrical potential records, Figure 7 (b). Closure forces with assumed magnitude and assumed distribution over the closed region were then used, in conjunction with the corresponding remote loading, to calculate the longitudinal strain (by numerical integration of Equation 16) at the gage location and compared with the experimental load-strain data. Typically, uniform closure forces (producing yield level stresses) were applied to a region near the crack tip, and rapidly decaying forces were applied to the remainder of the closed region. This distribution was considered to be consistent with the physical model. The forces that produced the best fit with the experimental data (Figure 19) were considered to be a reasonable estimate of the closure forces. Closure forces determined by this iterative procedure are shown in Table 3 and appear to be reasonable. A portion of the closure force at the crack tip may be regarded as a contribution from the compressive yield zone, although such an identification was not made explicitly. 
In arriving at these estimates, the distribution of closure forces was assumed to be constant in the thickness direction, and the crack front and the closure line were assumed to be straight and perpendicular to the specimen surface. Actually, the contact area would resemble that illustrated in Figure 20. This assumed three-dimensional distribution is quite reasonable, since the permanent tensile deformation near the surface is much larger than that in the center, because of the difference of plastic zone size (Figure 21). In this case, the distribution of the closure force must be solved as a three-dimensional problem. Because of this two dimensional approximation, the procedure for estimating the closure forces may be used only when the strain gage is located close to the crack tip.

\section{Computation of Crack Opening Displacement}

The relative displacement between several pairs of points in the longitudinal direction (that is, crack opening displacement) were computed by using the superposition model, and the magnitude and distribution of closure force estimated previously. The equations used in this computation are Equations 9, 11,13 , and 15.

The computed displacements are depicted in Figure 22. These curves are similar to those observed by Elber [10], Figure 1, and suggest that the onset of crack closure detected by several experimental techniques would be the same, Figure 23. 


\section{GENERAL DISCUSSIONS}

It has been shown that the crack tip stress intensity factor, $K$, defined by linear elasticity, is the most appropriate parameter for characterizing crack growth $[2,3,4]$. Because of a lack of understanding of the mechanism for fatigue crack growth, no fundamental fatigue crack growth laws have been proposed. The various "so-called" laws are empirical representations of available experimental data. In proposing the relationship given by Equation 1, Paris assumed that $\Delta K$ was the most significant parameter, and that stress ratio, $\mathrm{R}$, (or, $\mathrm{K}_{\mathrm{mean}}$; or $\mathrm{K}_{\mathrm{max}}$ ) is of only secondary importance.* In practical cases, the effect of $\mathrm{R}$ can be important. Attempts have been made to incorpor ate the influence of $R$ into the growth rate relations, again through empirical correlation $[5,6]$.

With the observation of crack closure, it is thought that a viable physical basis for explaining the influence of $\mathrm{R}$ on fatigue crack growth had been developed [10]. Because of closure, the effective stress intensity range, $\Delta K_{e f f}$, may differ considerably from the applied $\Delta \mathrm{K}$, and is a function of $\mathrm{R}$. Elber showed that good agreement with experimental data was obtained on this basis. Unfortunately, several questions remain unresolved. Firstly, Elber argued that as soon as crack closure begins, the singularity at the crack tip is removed. Thus, the corresponding $K$ value at the onset of crack closure is to be regarded as the $\mathrm{K}_{\mathrm{min}}$ in the load cycle; that is, a truncation of the lower portions of the load cycles. Experimental results from this investigation show the strains ahead of the

* Environmental effects will not be considered in this discussion. 
crack tip continue to decrease with unloading beyond this point, Therefore, since fatigue damage is related to the cyclic strain range, the effective $K_{\min }$ is likely to be somewhat lower than the level at the onset of closure (Figure 24). These differences in viewpoint cannot be resolved by the approximate two-dimensional model developed in this report. Secondly, because of the preliminary nature of Elber's closure data (see Introduction), the correlation between the rate of fatigue crack growth and $\Delta K_{\text {eff }}$ given by Equation 5 requires additional ver ification . Recent data obtained by FitzGerald and Wei [31] indicate that there is an effect of $R$ on fatigue crack growth, for $R$ values from 0.05 to 0.9 , in the Ti-6Al-4V alloy used in this study, Figure 25. The present results indicate the absence of closure at $R$ greater than 0.3 . Hence, crack closure is likely to be only one of several factors that contribute to the observed $R$ effect.

The importance of delay, or retardation in the rate of fatigue crack growth, produced by load interactions in variable-amplitude loading on the accurate prediction of fatigue lives has been well recognized for some time $[32,33,34]$. Jonáš and Wei [35] showed that the phenomenon of delay is very complex and can depend on a broad range of loading variables. Due to a lack of physical and phenomenological understanding of the effects of load interactions on fatigue crack growth, no successful mode! has been proposed to account for delay. In general, delay effects have been attributed to such things as crack tip blunting [36], residual stress ahead of the crack tip [34], and more recently, crack closure proposed by Elber [10]. The first two of these theories do not lend themselves to direct experimental observation. The third, crack closure 
proposed by Elber, may be verified experimentally by determining if delay occurs in the absence of closure. For a loading spectrum depicted on Figure 26 , there should not be any crack closure occurring for $K_{m i n}$ equal to or greater than $12 \mathrm{ksi} \sqrt{\mathrm{in}},(\mathrm{R}>0.3)$, according to the result of the last section. However, significant amount of delay was observed, Figure 26. This experimental result shows again that crack closure cannot be regarded as the sole cause for delay.

The present series of experiments have shown that crack closure can and does occur. Its occurrence is dramatically evidenced by changes in electrical potential, strain and crack opening displacement with load. However, crack closure cannot account for all of the observed fatigue behavior. Additional research is needed to better establish its significance. 


\section{CONCLUSION}

The following conclusions can be made on the basis of the experimental results on the Ti-6Al-4V alloy:

1. Crack closure does occur during fatigue. For the Ti-6Al-4V alloy, closure was observed at stress ratios, $R$, between 0.05 and 0.3 .

2. The extent of closure can be determined by electrical-potential measurement method with reasonable accuracy.

3. The stress intensity factor at the onset of closure depends on the stress ratio, $R$, and on $K_{\max }$.

4. Onset of closure can be detected by measurements of such quantities as electrical potential, crack-opening displacement, and strain.

5. The closure force near the crack tip was estimated to produce yield stresses. A part of this estimated closure force may be attributed to the plastic zone.

6. Crack closure cannot be regarded as the sole cause for the various observed phenomena for fatigue. For example, it cannot completely account for the influence of stress ratio, $R$, on fatigue crack growth; neither can it fully explain the delay phenomenon under variable-amplitude loading. 


\section{TABLE 1}

Chemical Composition and Tensile Properties

(Reactive Metal Ingot No. 293831, Lot 05)

\section{Chemical Composition - Weight Percent} (Ingot Analysis)

$\begin{array}{cccccccc}\underline{\mathrm{C}} & \underline{\mathrm{N}} & \underline{\mathrm{Fe}} & \underline{\mathrm{Al}} & \underline{\mathrm{V}} & \underline{\mathrm{O}} & \underline{\mathrm{H}(\mathrm{ppm})} & \underline{\mathrm{Ti}} \\ 0.03 & 0.013 & 0.13 & 6.2 & 4.2 & 0.12 & 90 & \text { Balance }\end{array}$

Tensile Properties*

Direction

\begin{tabular}{lcc} 
Yield Strength & Tensile & Elongation \\
Strength & in 2 in. \\
$\mathrm{ksi}\left(\mathrm{MN} / \mathrm{m}^{2}\right)$ & $\mathrm{ksi}\left(\mathrm{MN} / \mathrm{m}^{2}\right)$ & percent \\
\hline
\end{tabular}

Transverse

(Average)

$149.5(1,031) \quad 152.6(1,052)$

12.5

$149.8(1,033) \quad 153.4(1,058)$

12.5

$\underline{148.3}(1,023) \quad \underline{151.4}(1,044)$

11. 7

$149.2(1,029) \quad 152.5(1,051)$

12. 2

Longitudinal

\begin{tabular}{llll} 
& $151.1(973)$ & $151.0(1,041)$ & 11.7 \\
(Average) & $\underline{141.9}(978)$ & $\underline{151.8}(1,047)$ & $\underline{12.5}$ \\
\hline $141.5(976)$ & $151.2(1,042)$ & 12.0
\end{tabular}

* Production annealed $1,450^{\circ} \mathrm{F}, 15 \mathrm{~min} .+$ air cool. 
TABLE II

Strain Gage Position

(inch)

\begin{tabular}{|c|c|c|c|c|}
\hline Position & $\begin{array}{c}\text { Gage } \\
\mathrm{A}\end{array}$ & $\begin{array}{c}\text { Gage } \\
\mathrm{B}\end{array}$ & $\begin{array}{c}\text { Gage } \\
\mathrm{C}\end{array}$ & $\begin{array}{c}\text { Gage } \\
\mathrm{D}\end{array}$ \\
\hline $\mathrm{X}$ & 0.347 & 0.496 & 0.644 & 0.797 \\
$\mathrm{Y}$ & 0.036 & 0.083 & 0.036 & 0.079 \\
\hline
\end{tabular}

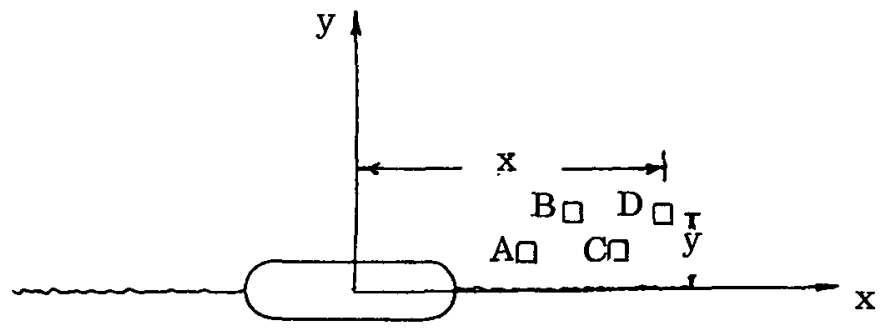




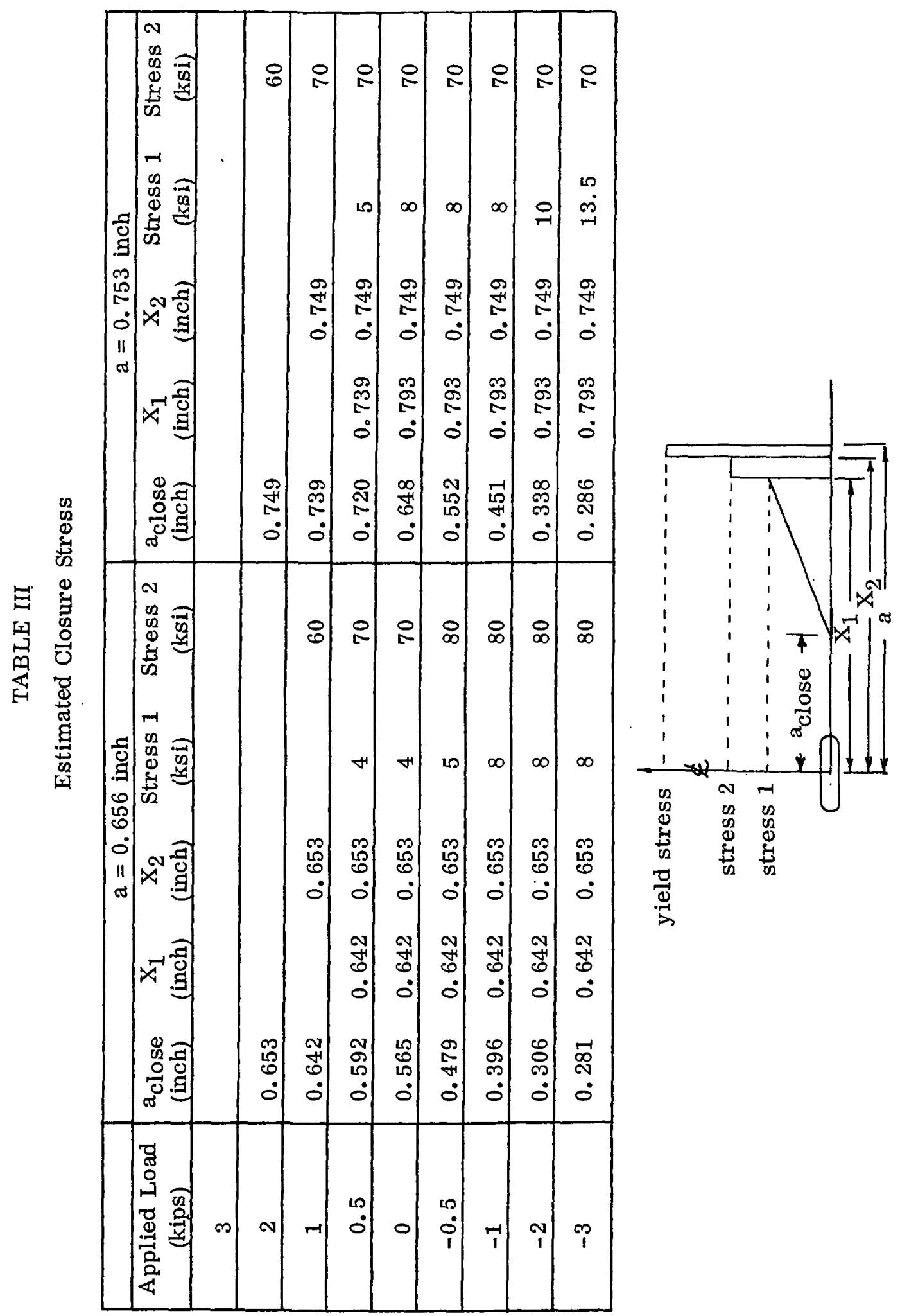




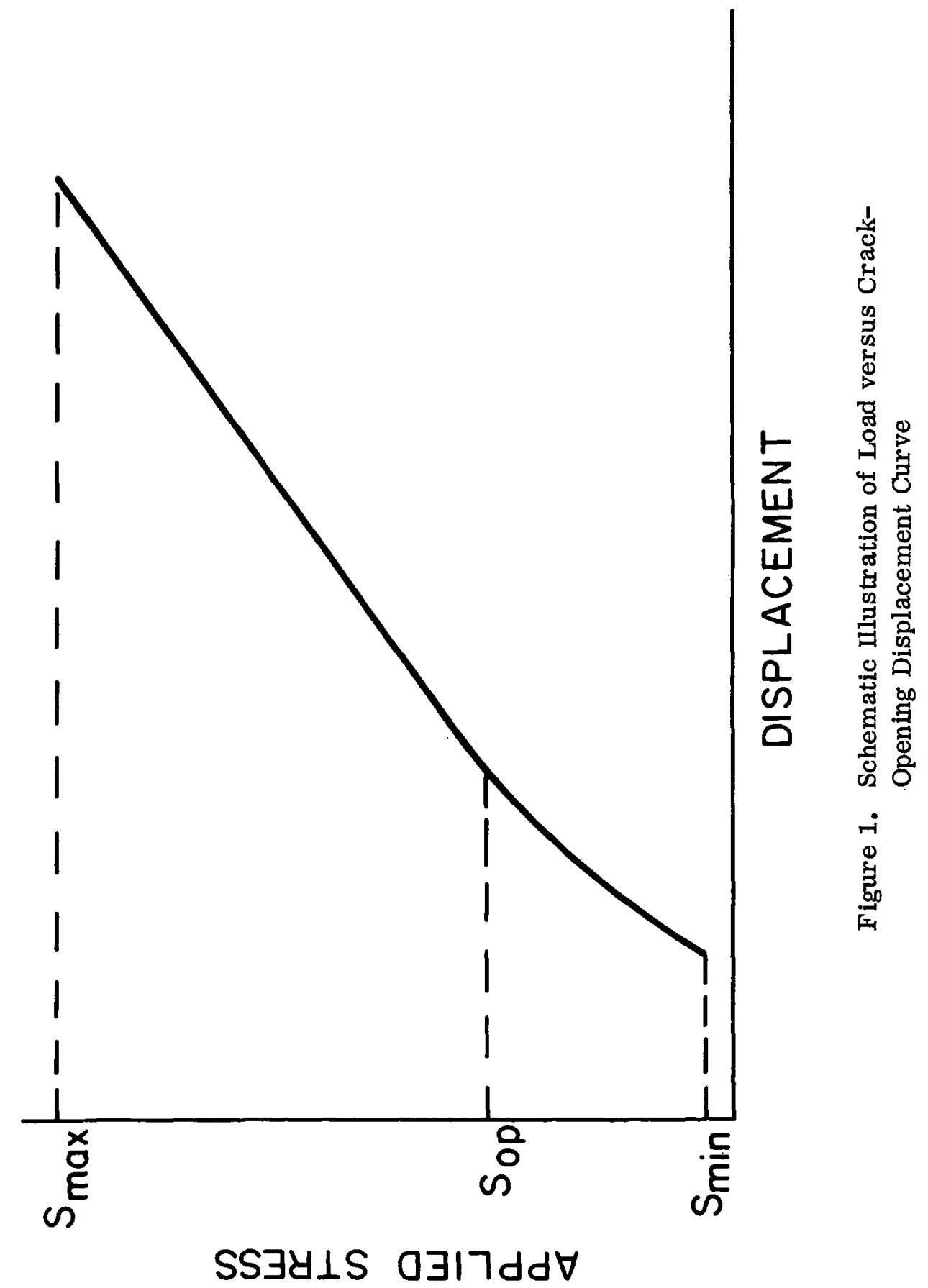




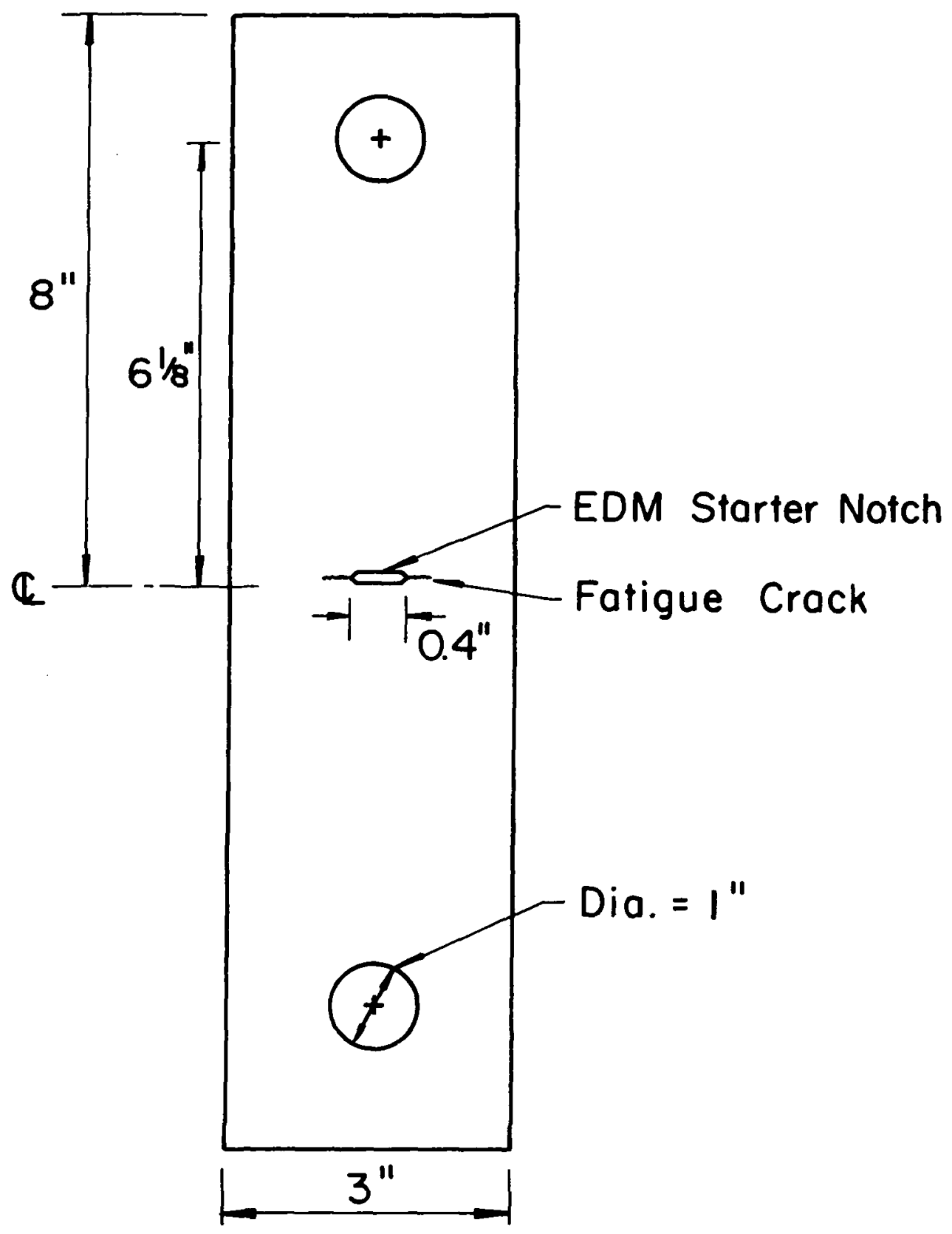

Figure 2. Center-Cracked Specimen 


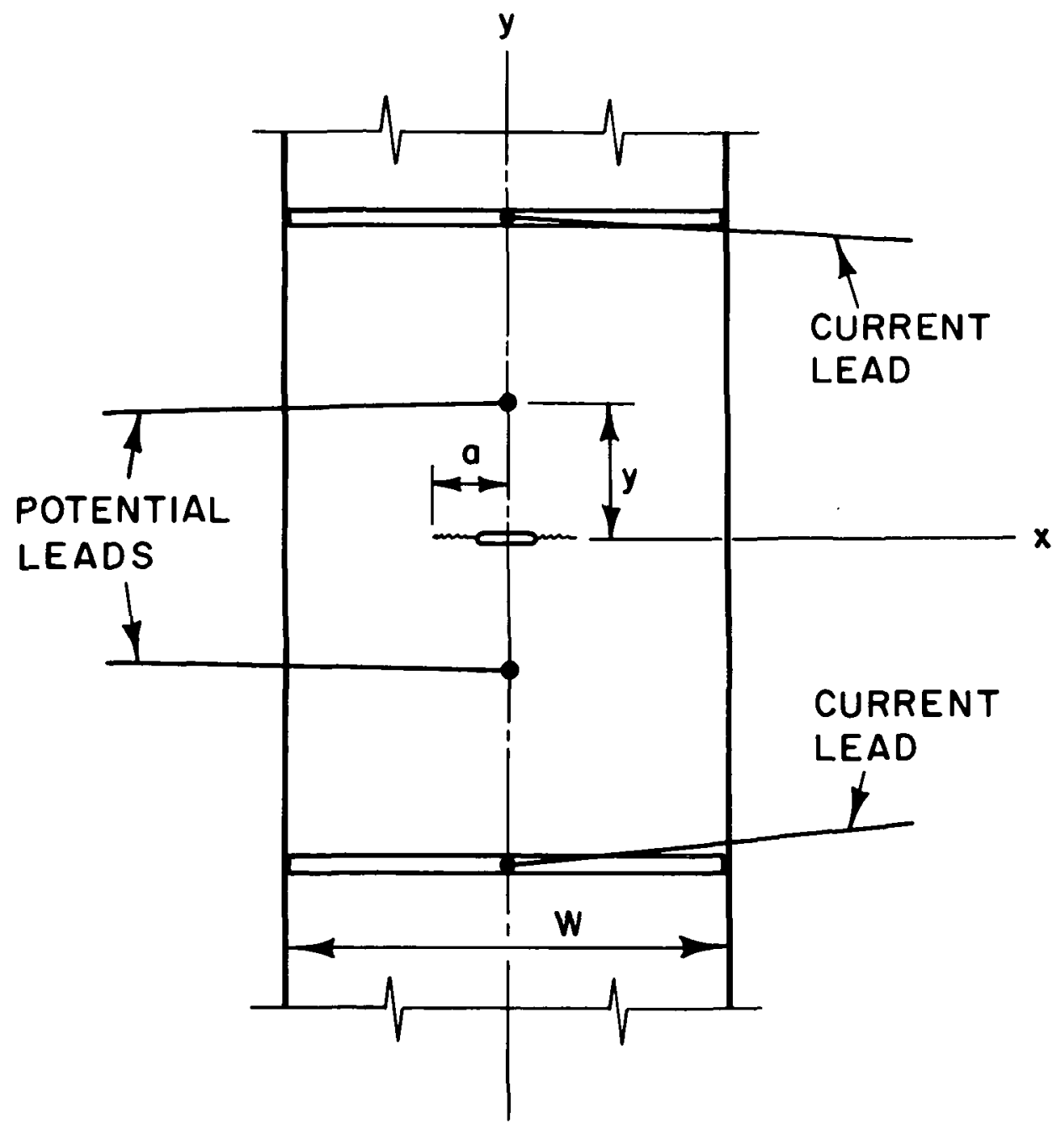

Figure 3. Potential Leads on a Center-Cracked Specimen 


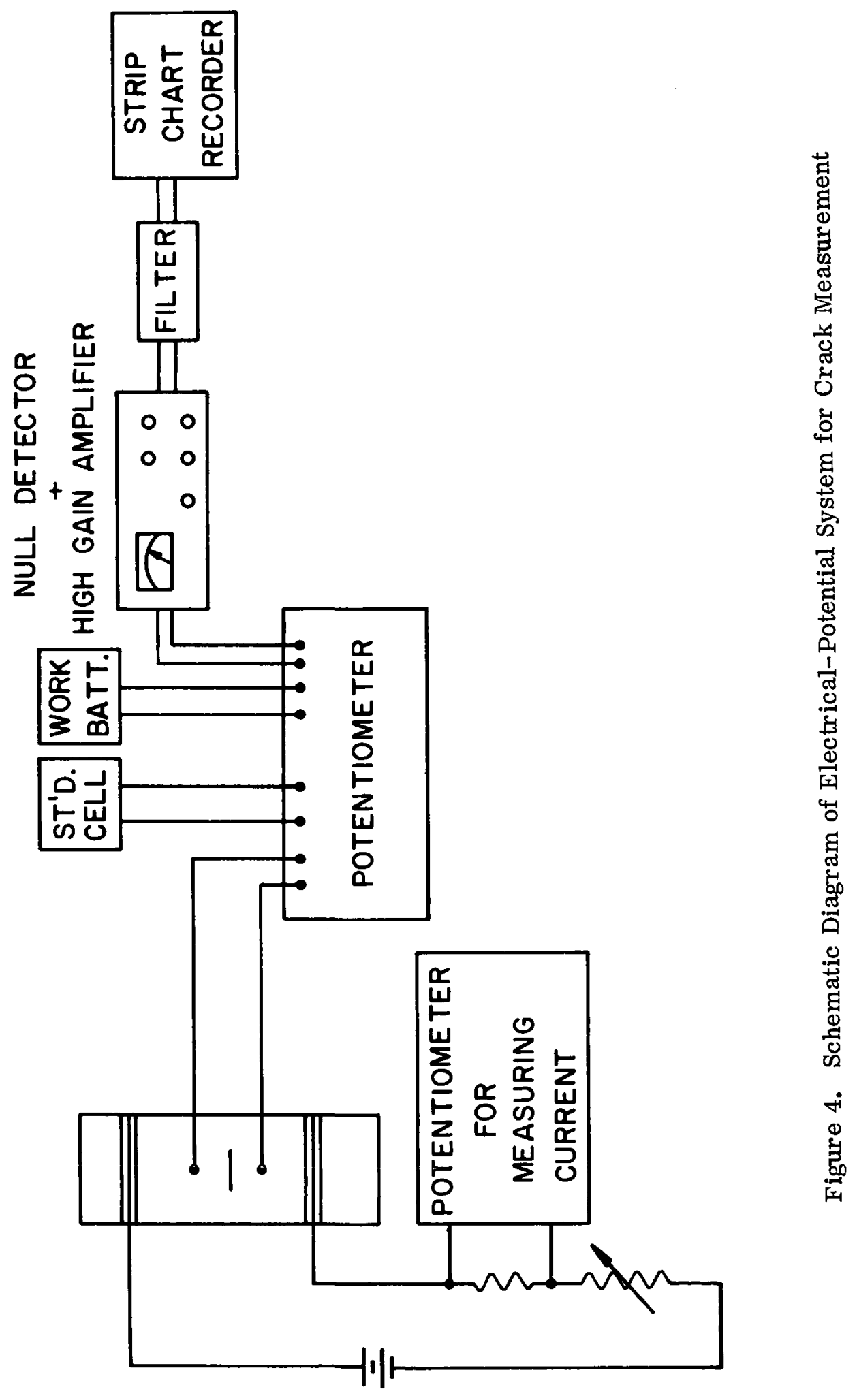




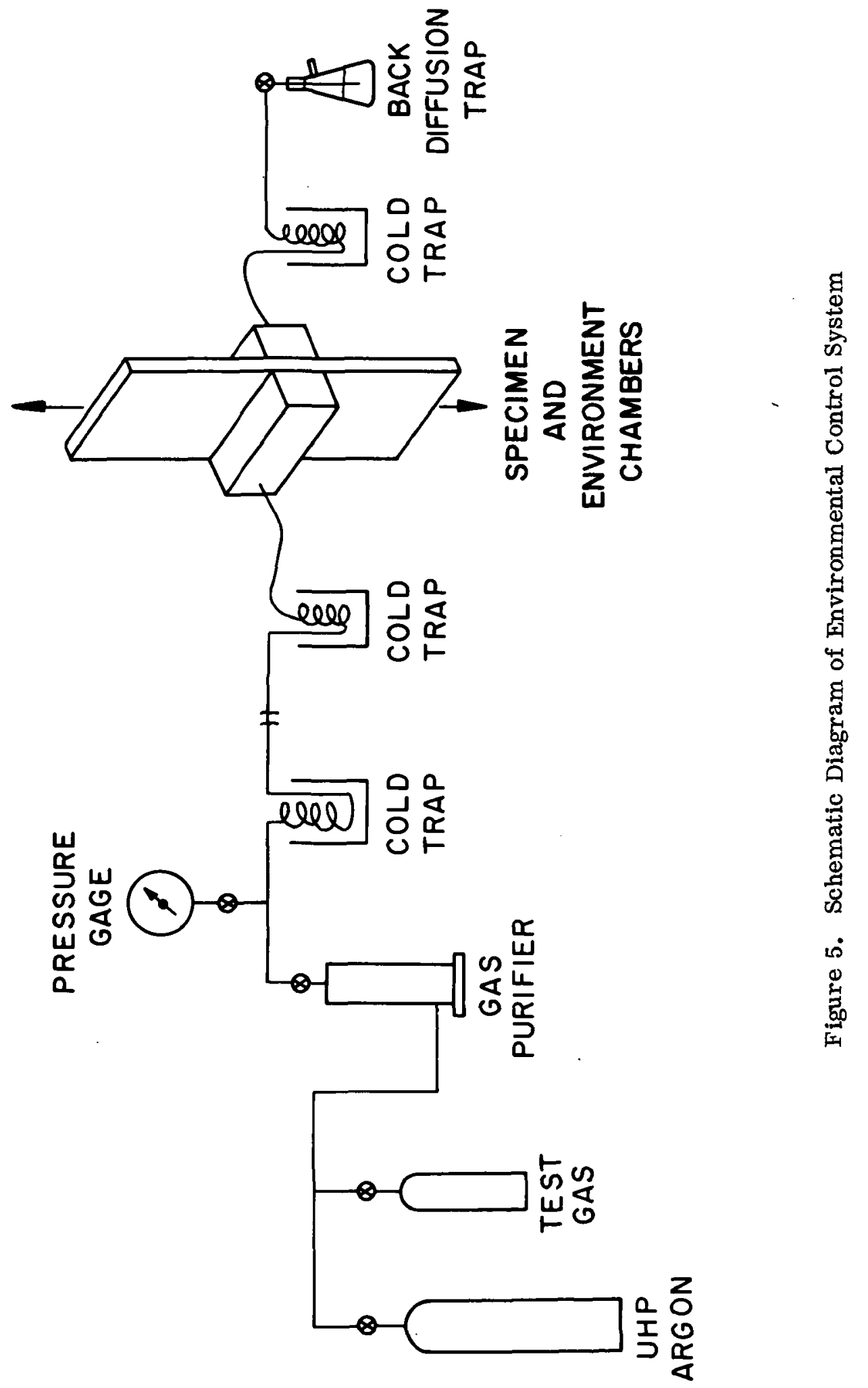




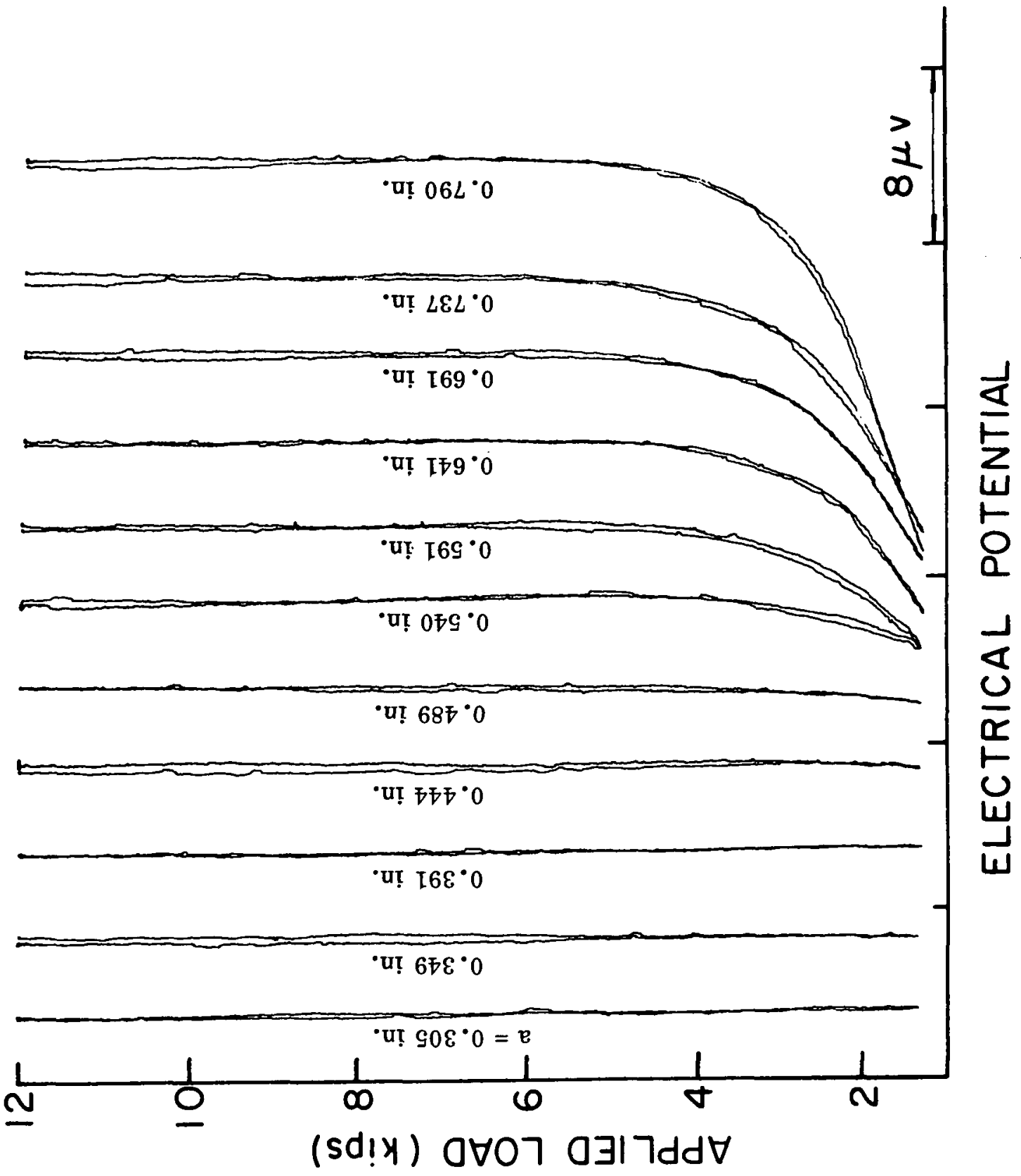

Figure 6a. Load versus Electrical Potential Curves (before the Compressive Loading Cycle) $(\mathrm{R}=0.1)$ 


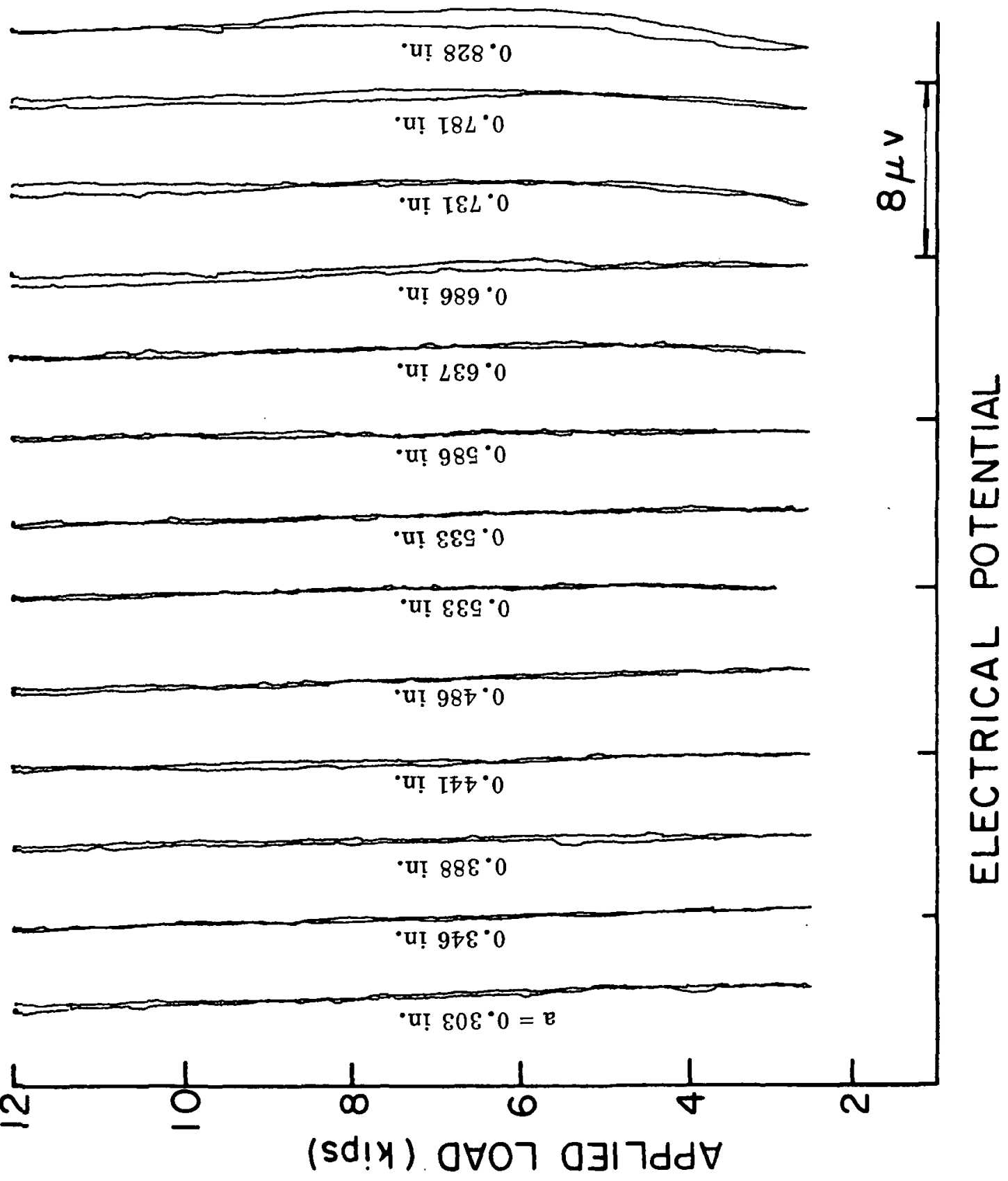

Figure 6b. Load versus Electrical Potential Curves (before the Compressive Loading Cycle) $(R=0.2)$ 

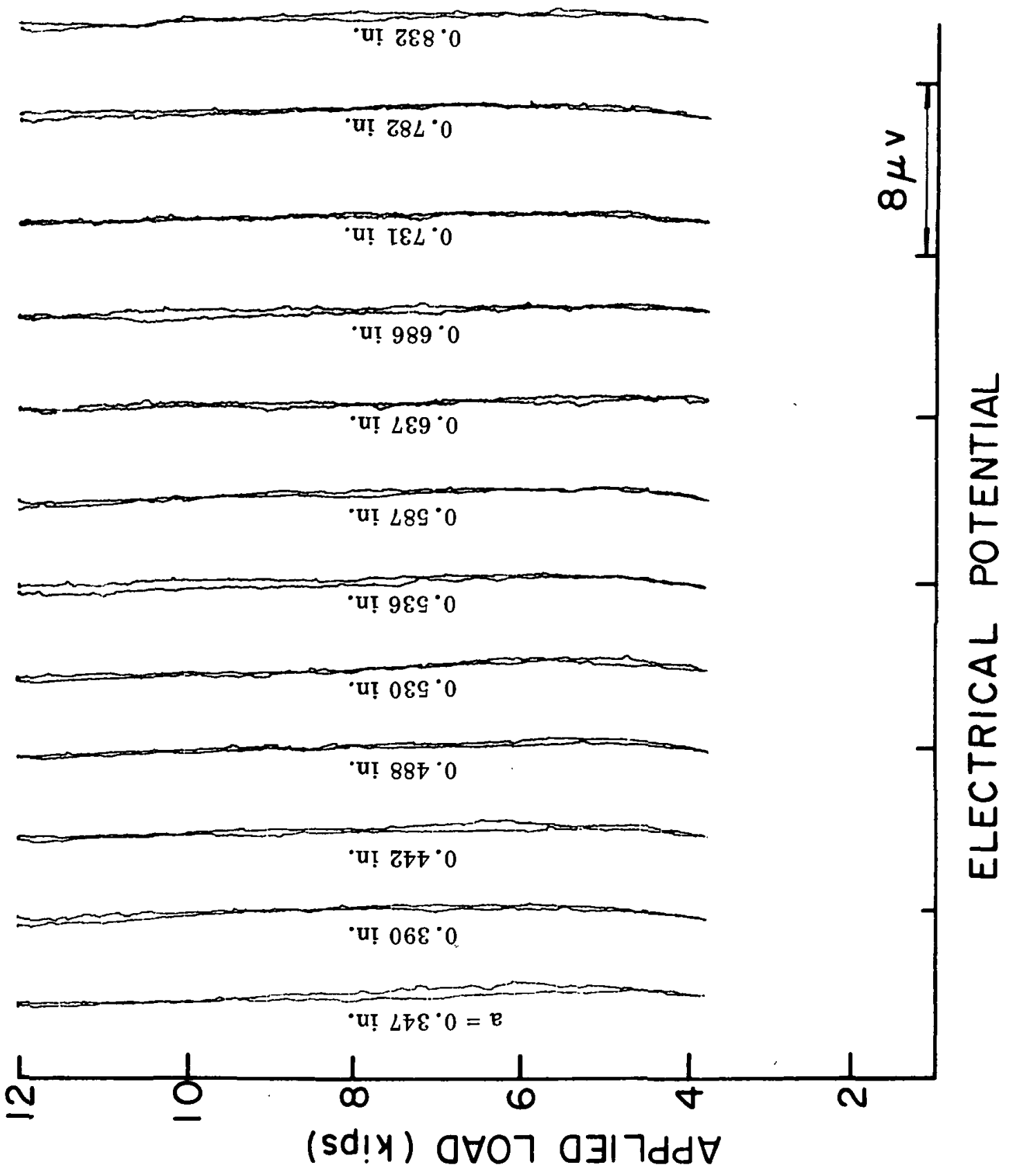

Figure 6c. Load versus Electrical Potential Curves (before the Compressive Loading Cycle) $(\mathrm{R}=0.3)$ 


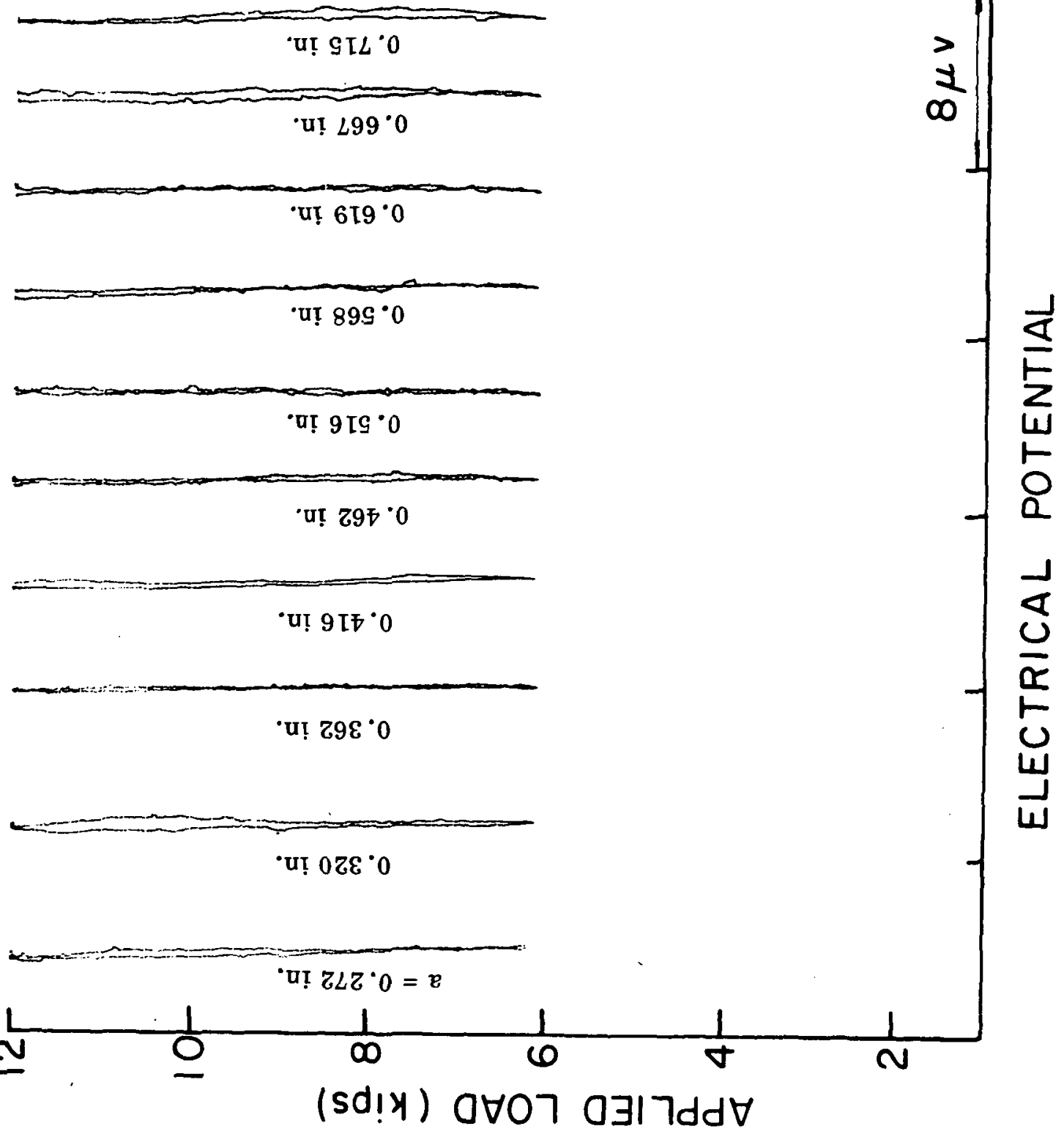

Figure 6d. Load versus Electrical Potential Curves (before the Compressive Loading Cycle) $(R=0.5)$ 


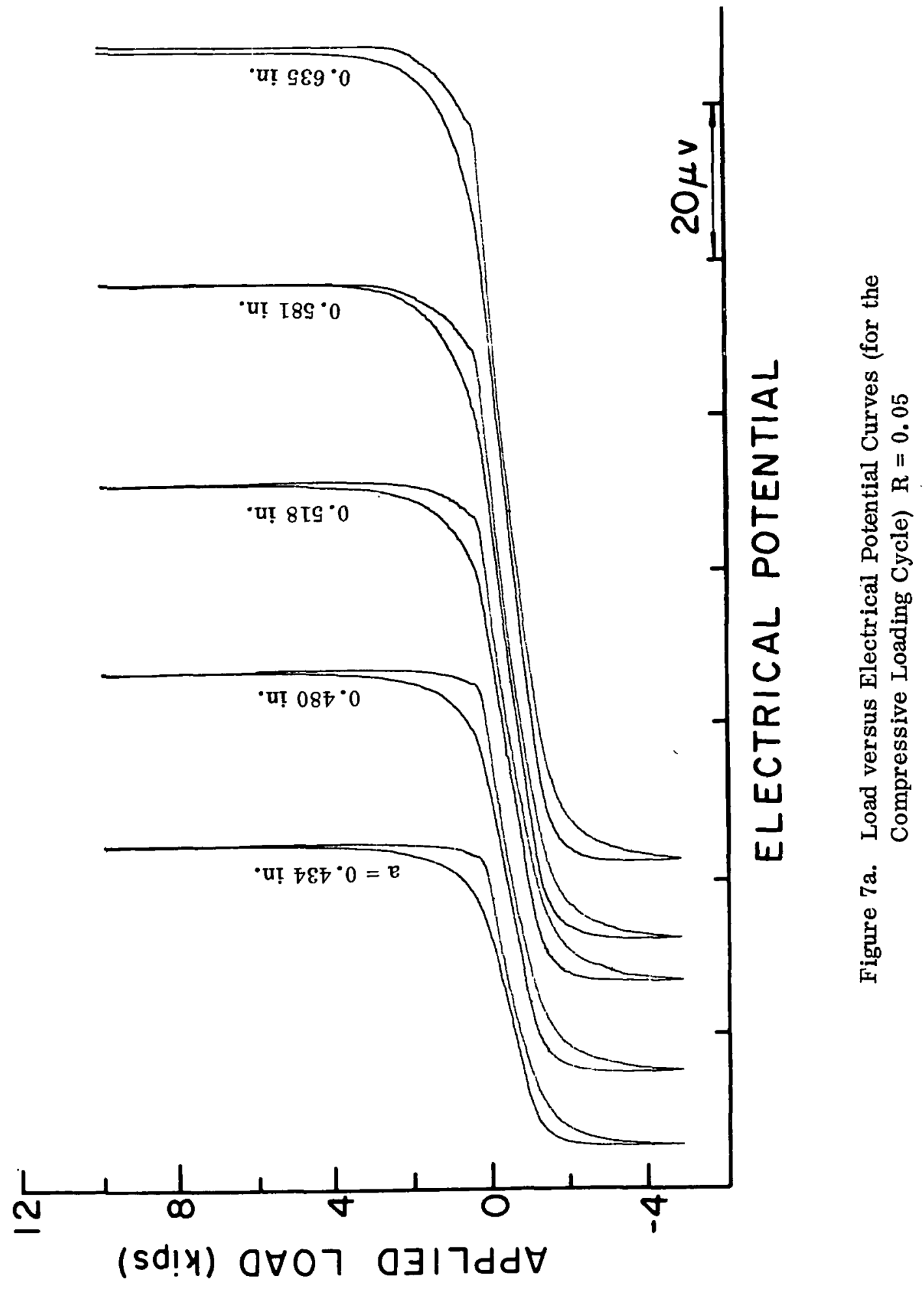




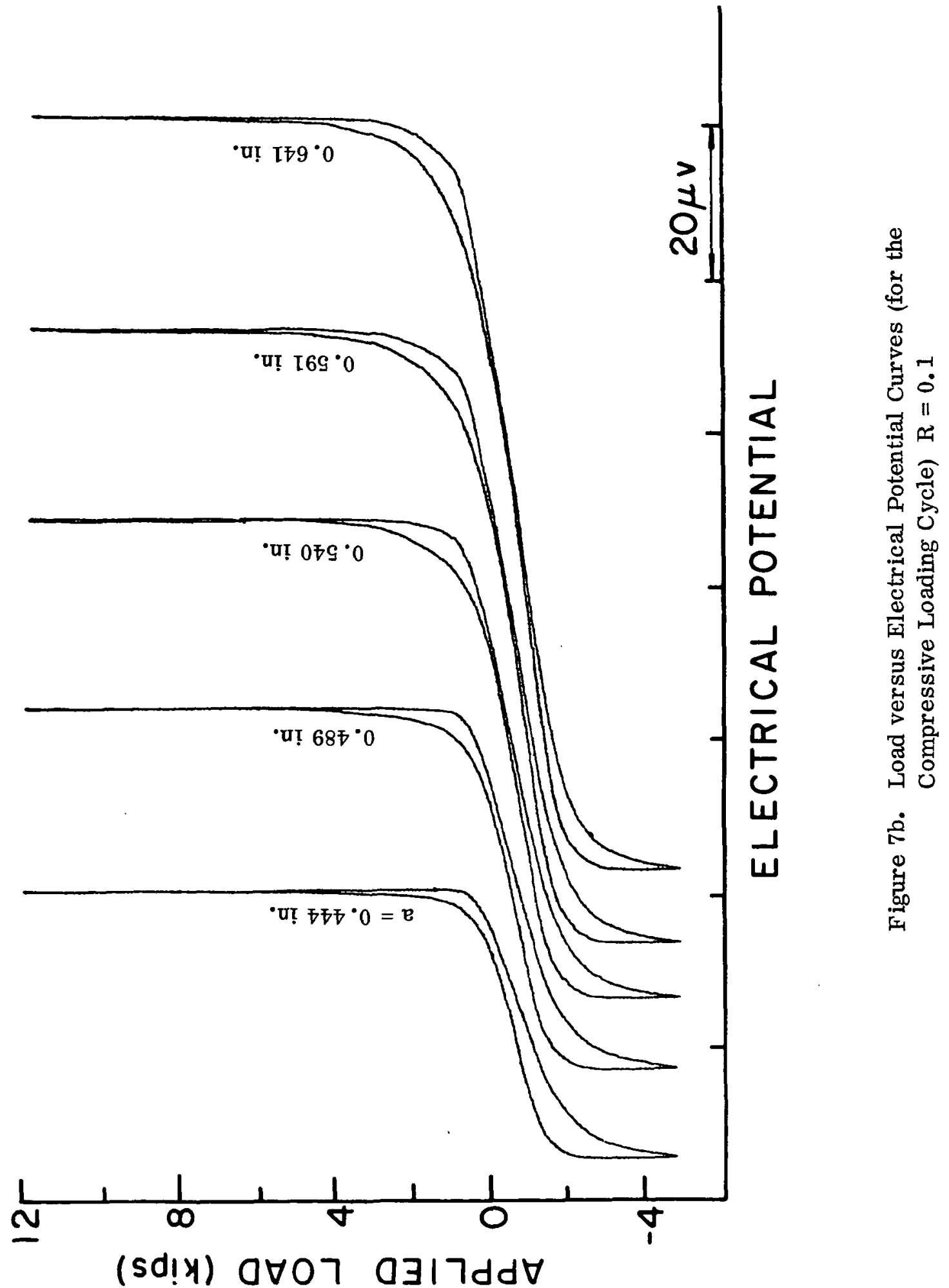




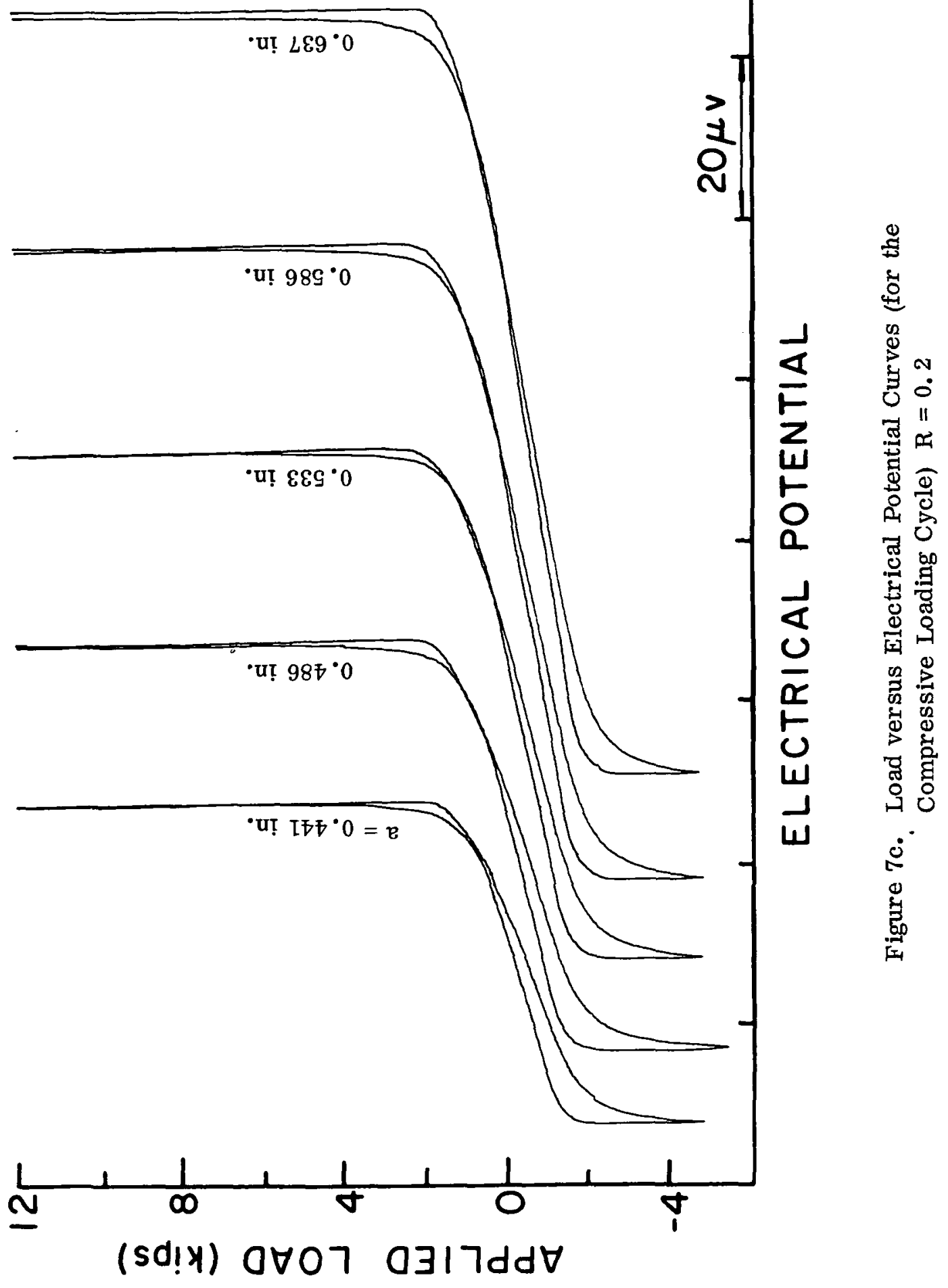




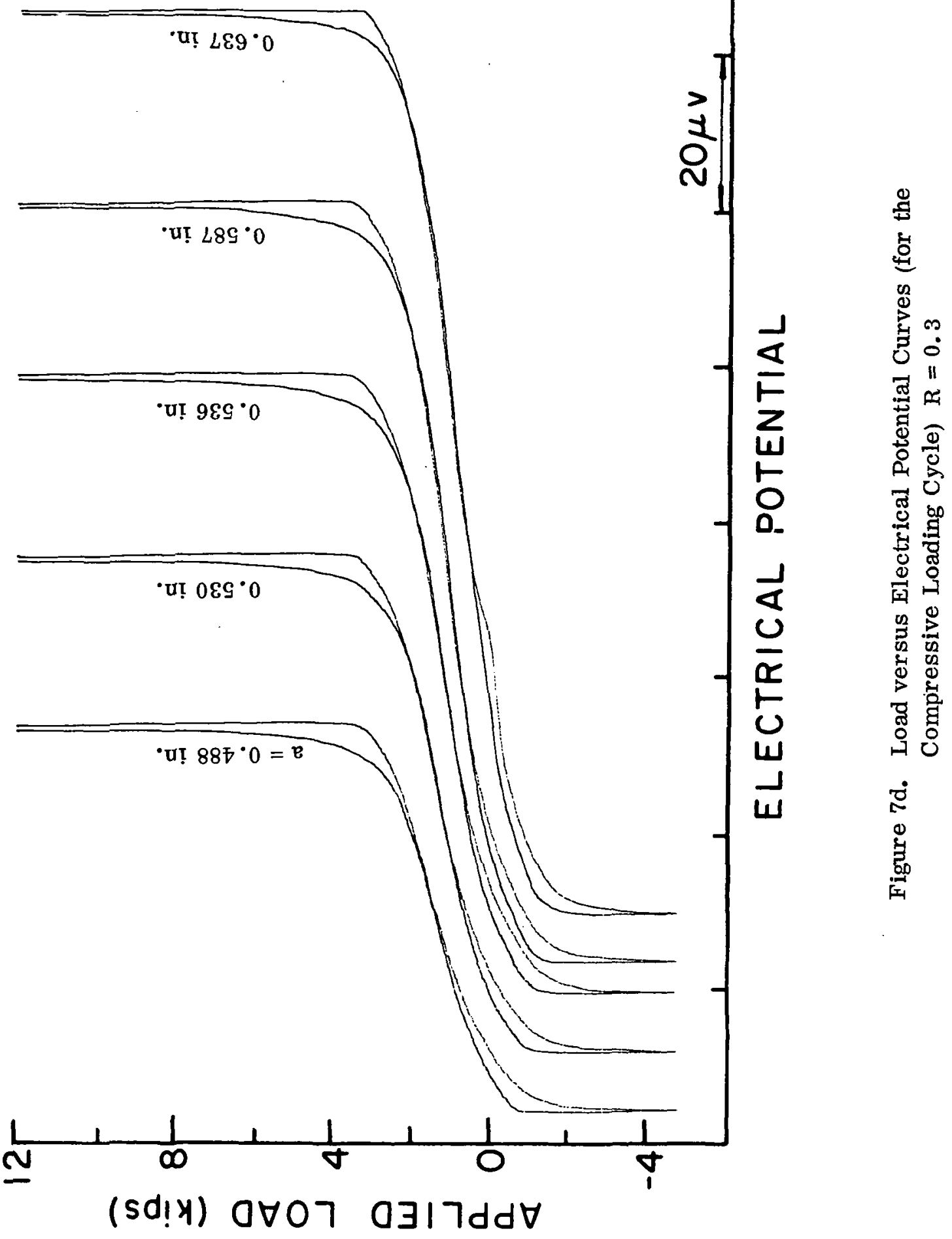




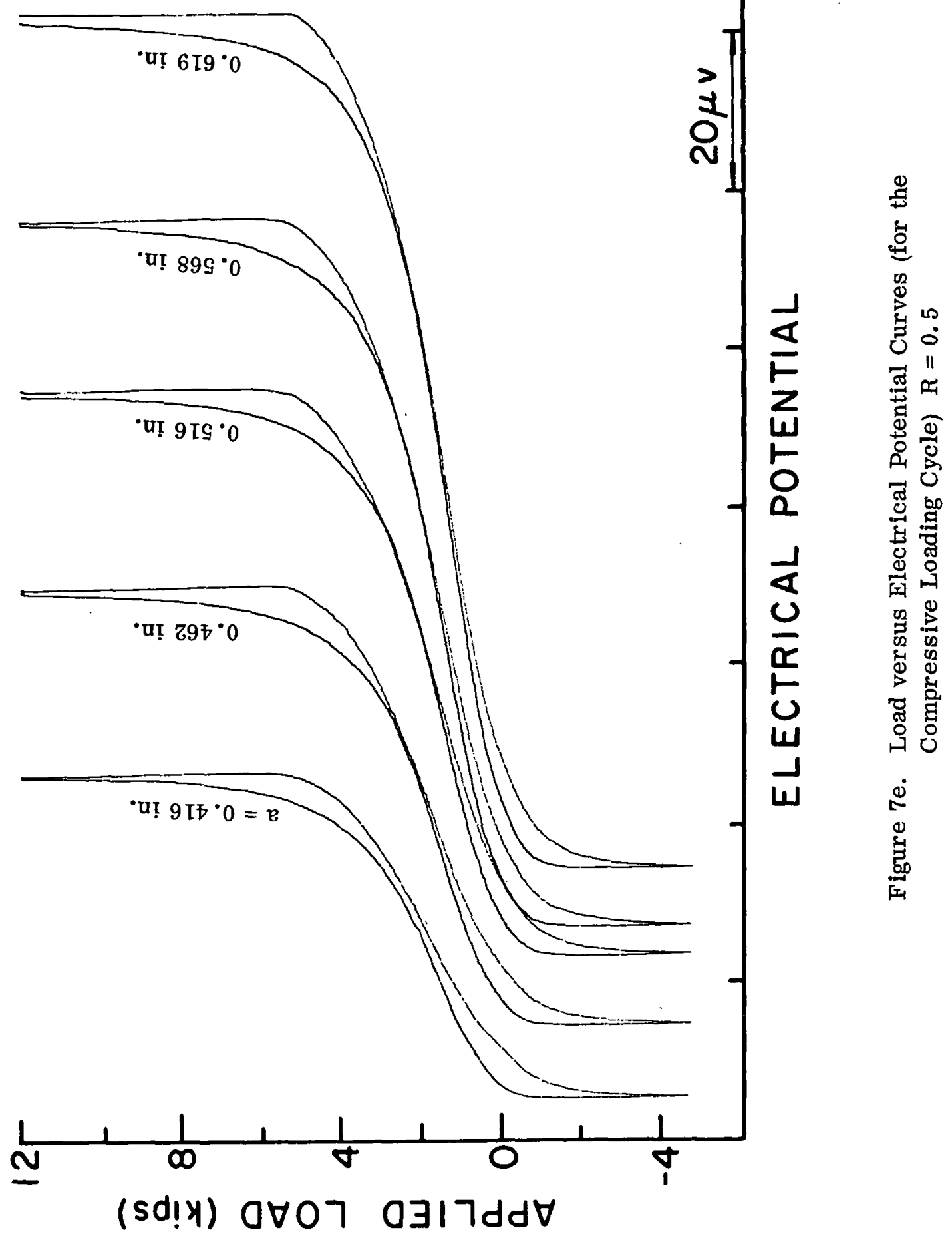



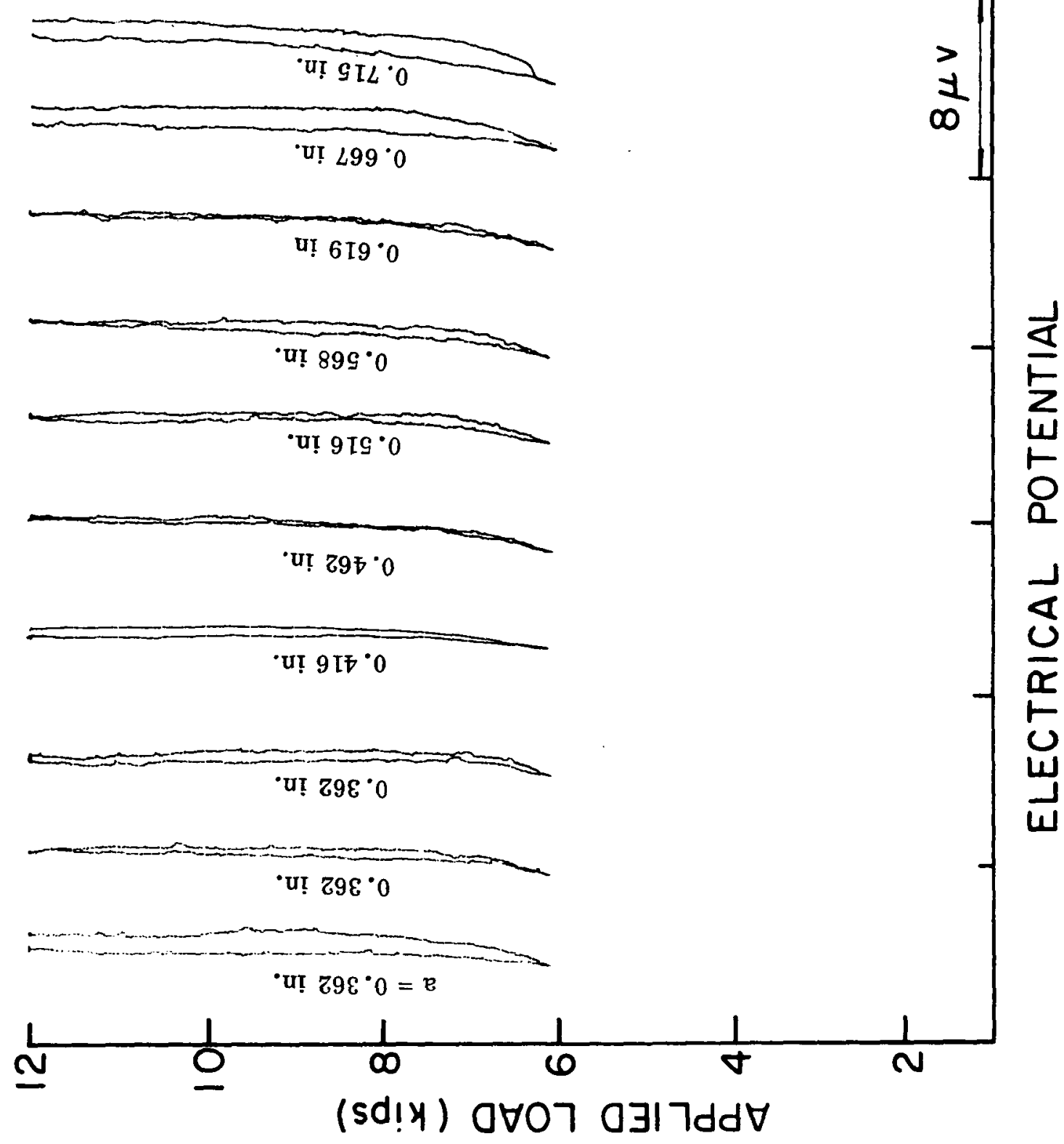

Figure 8. Load versus Electrical Potential Curves (after the Compressive Loading Cycle) 


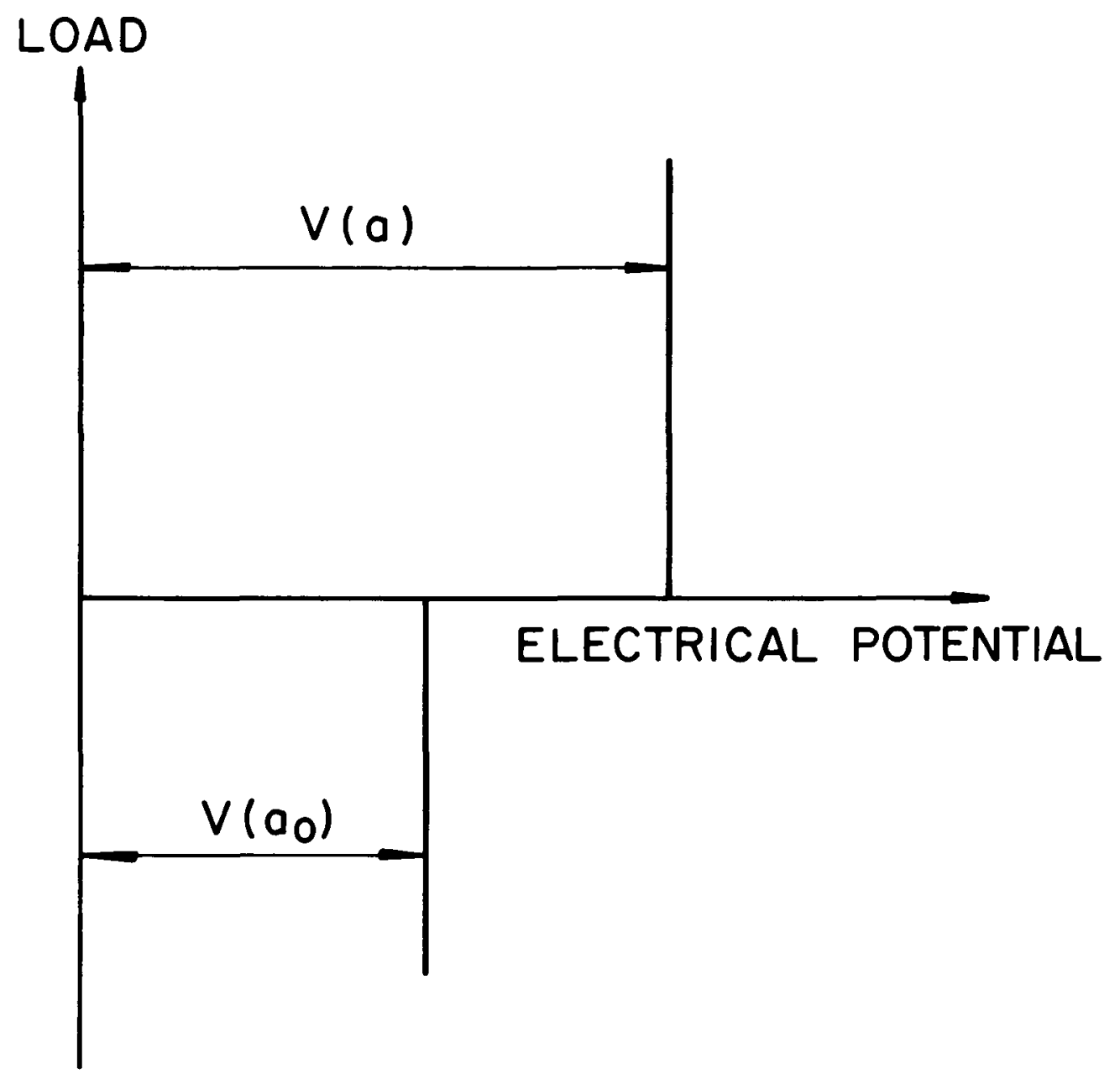

Figure 9. Load versus Electrical Potential Curve for an Idealized Crack in an Elastic Medium 

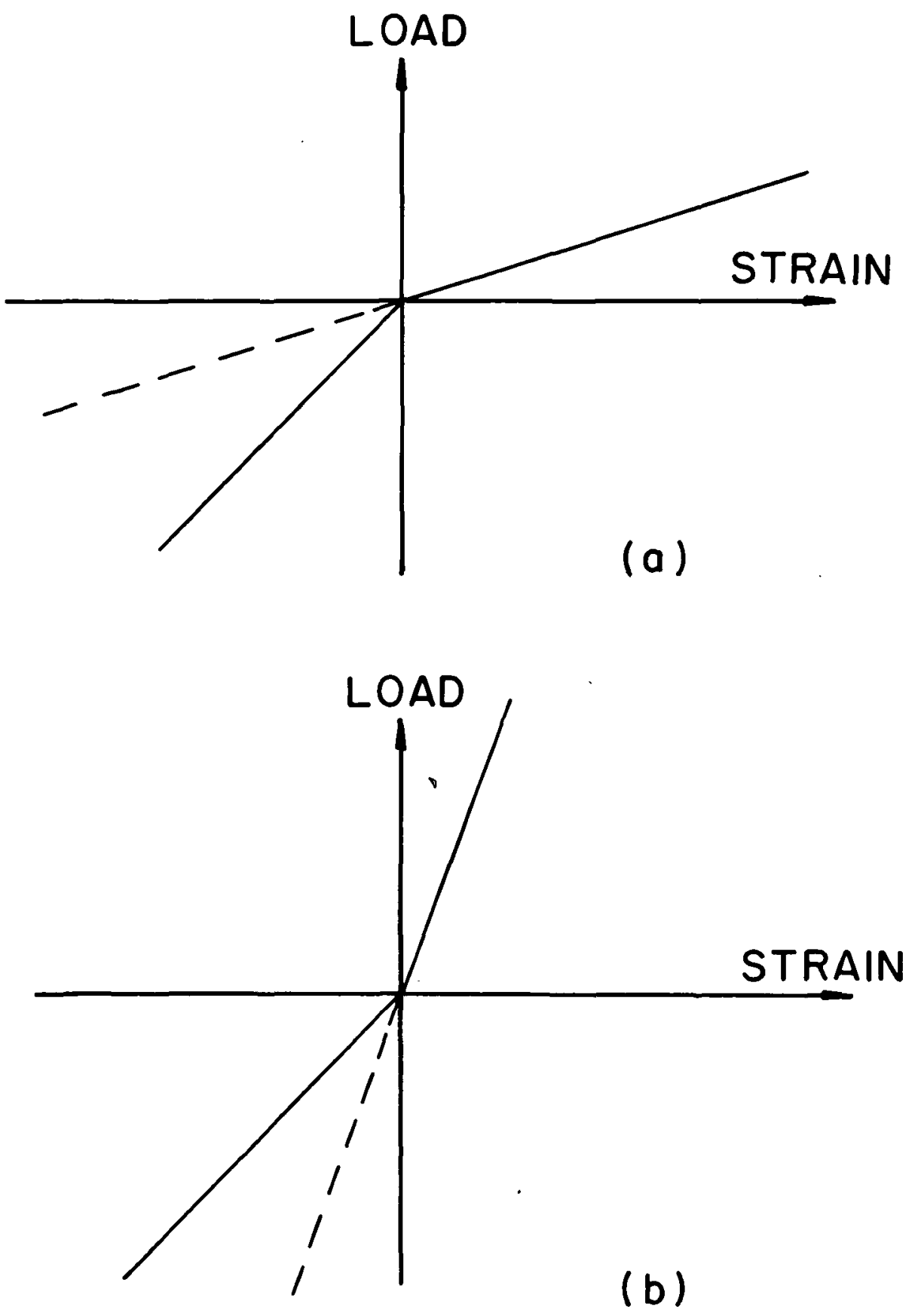

Figure 10. Load versus Strain Curve for an Idealized Crack in an Elastic Medium

(a) Strain Gage Located Ahead of the Crack Tip

(b) Strain Gage Located Behind the Crack Tip 


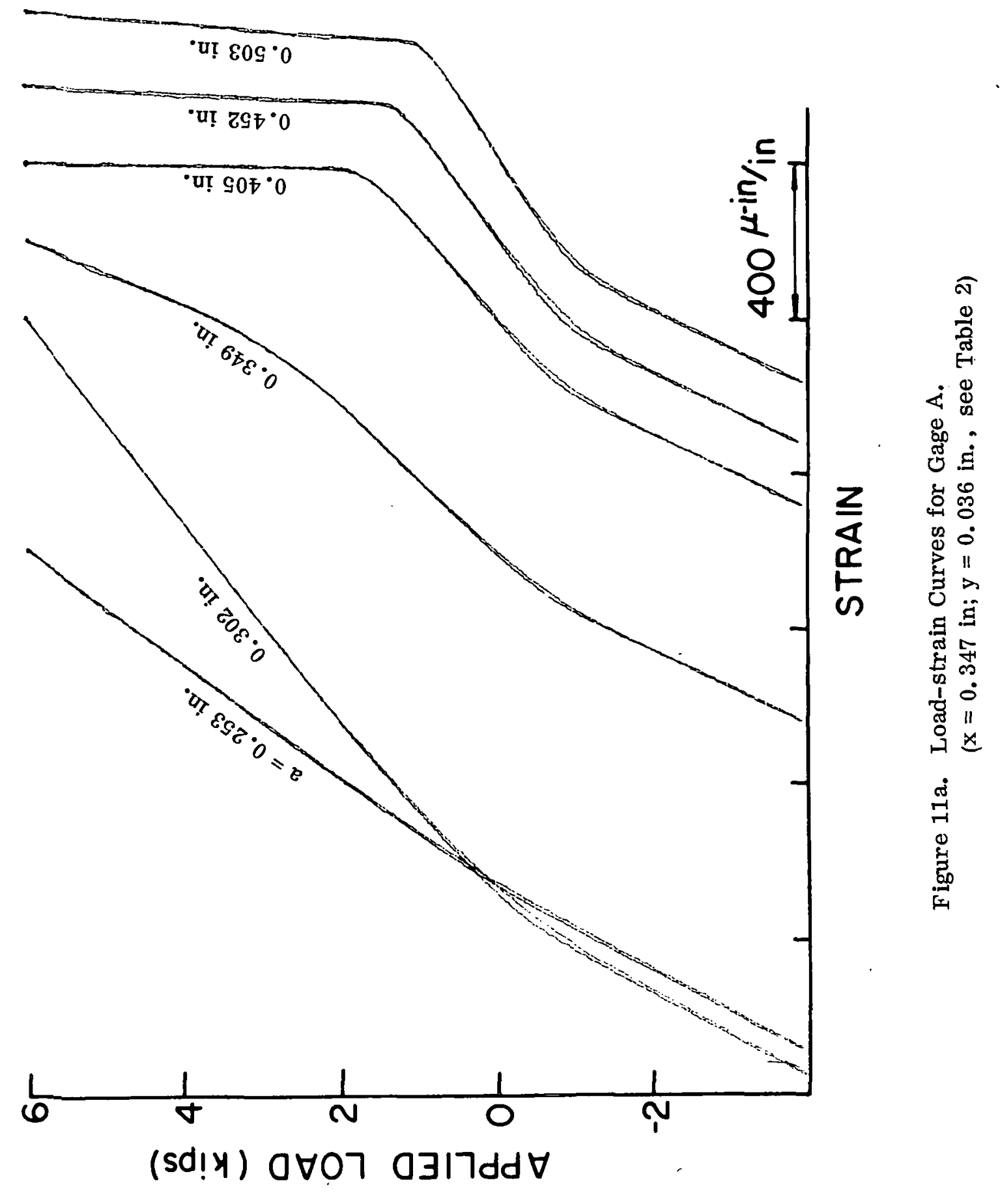




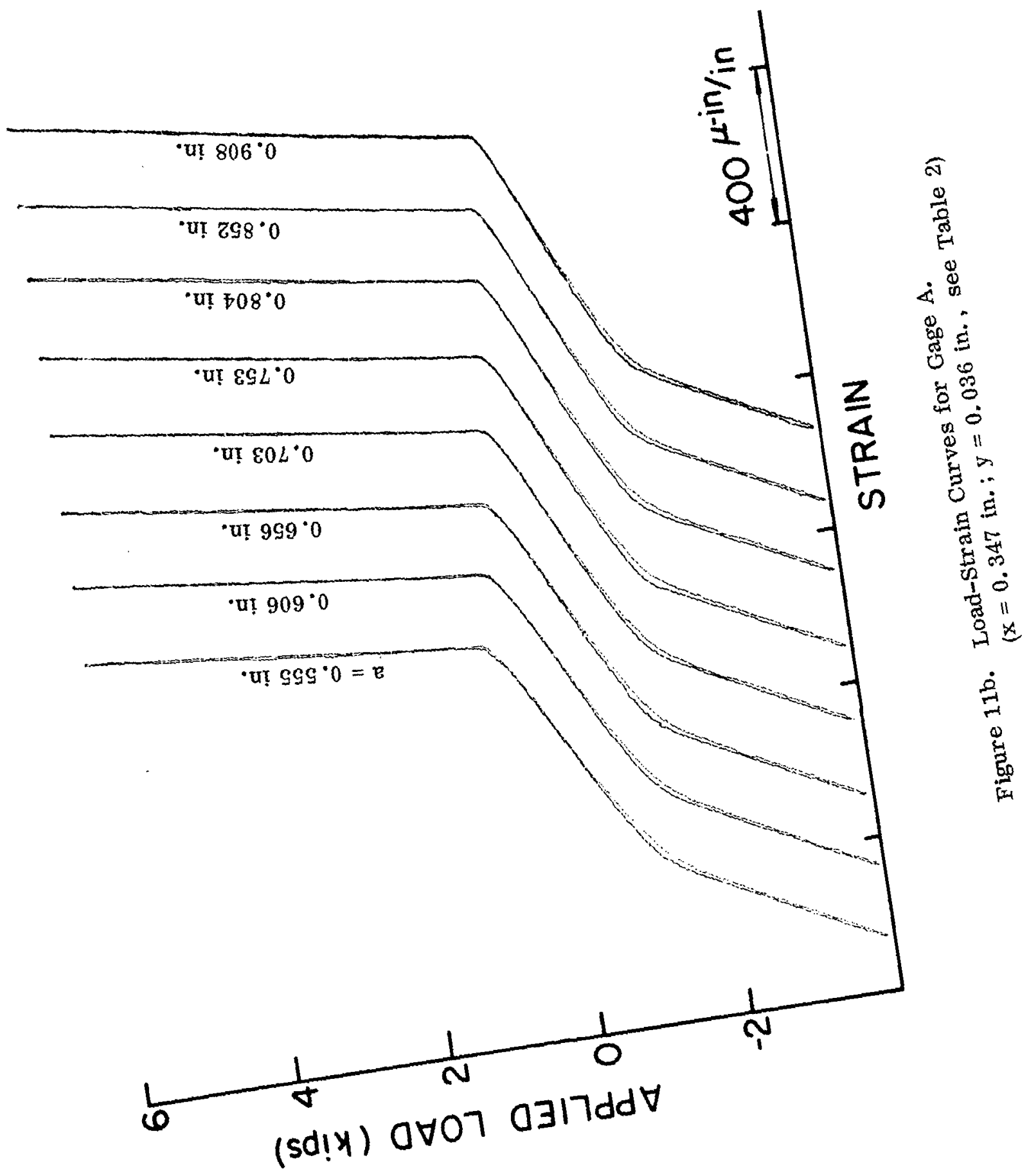




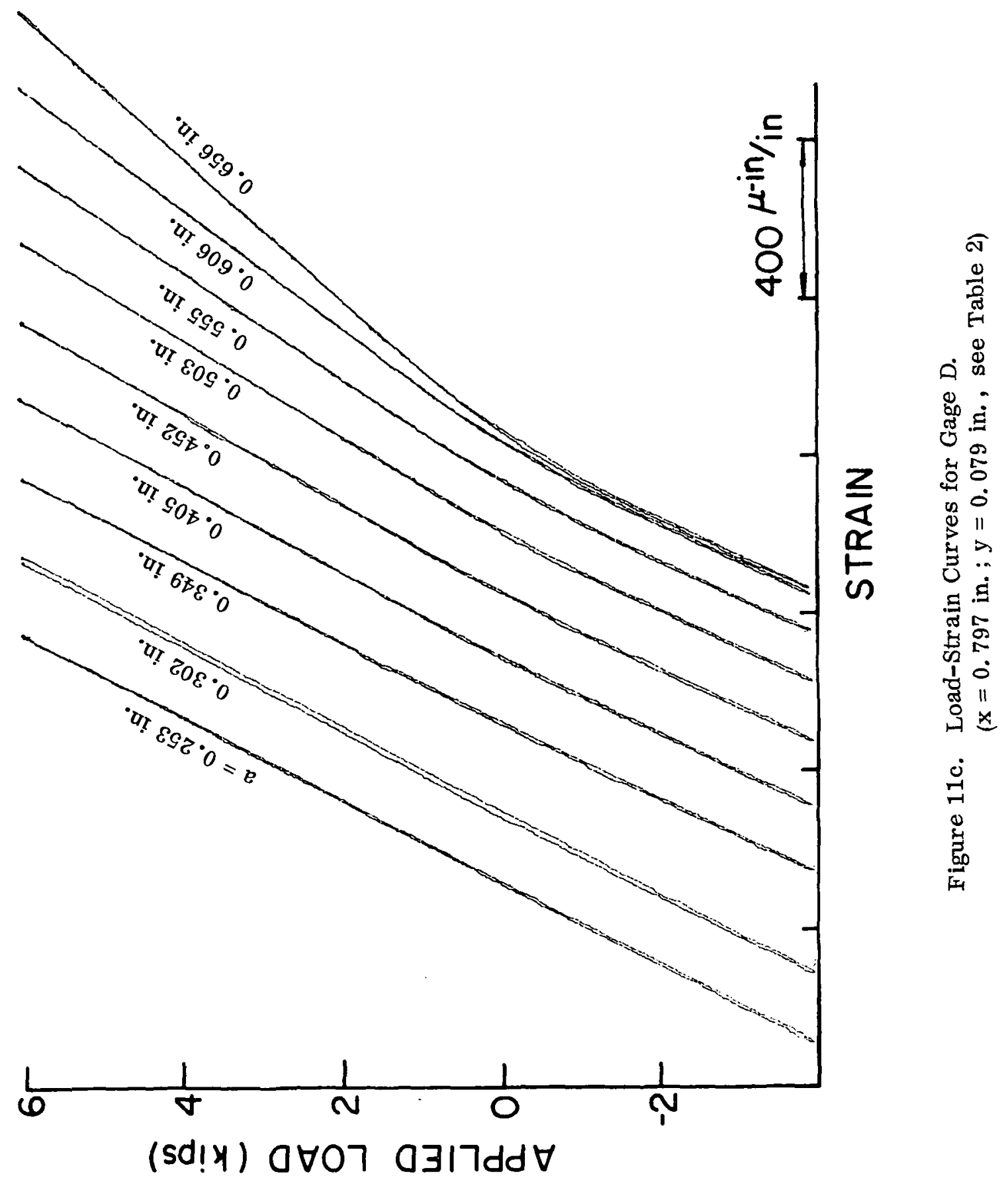




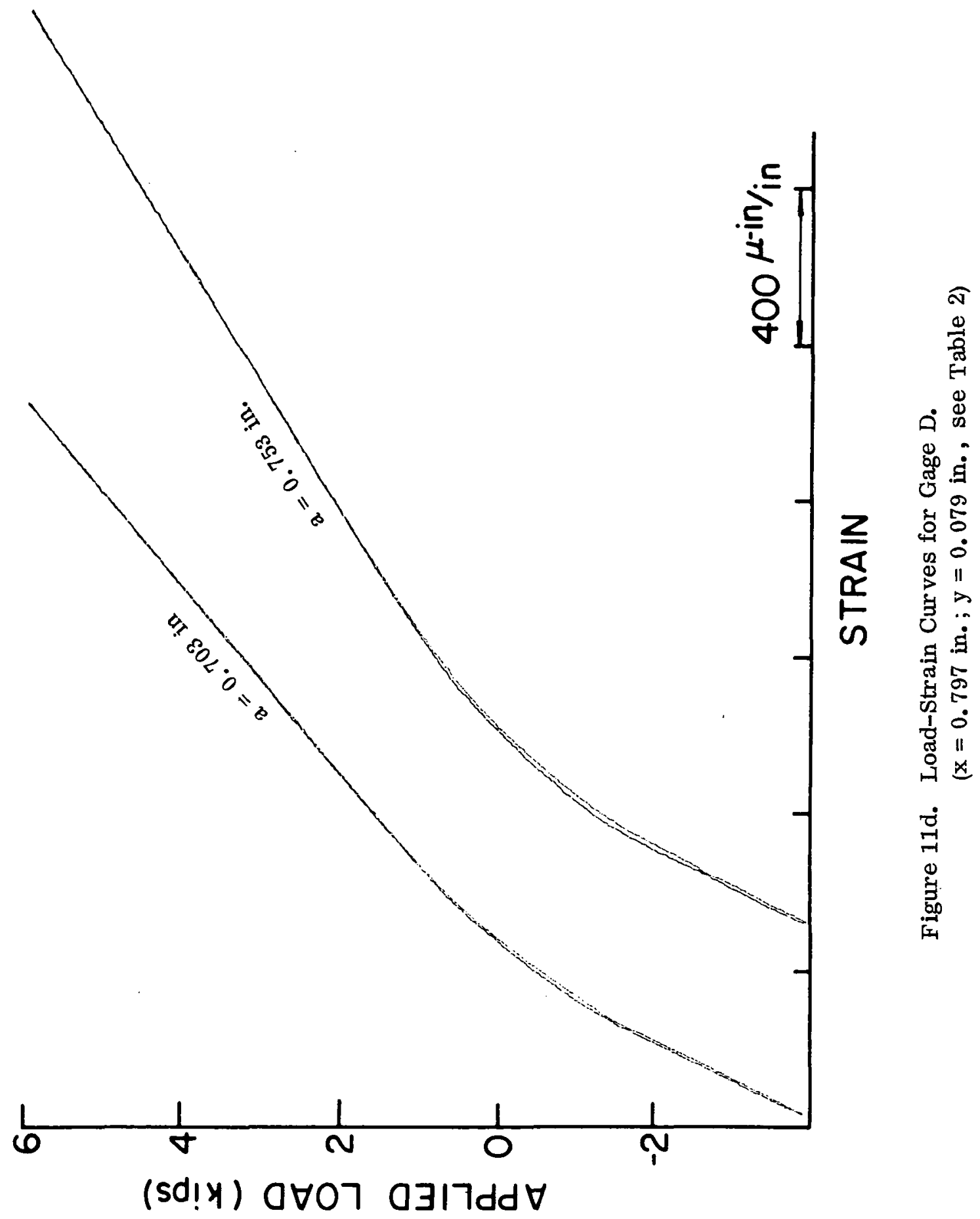




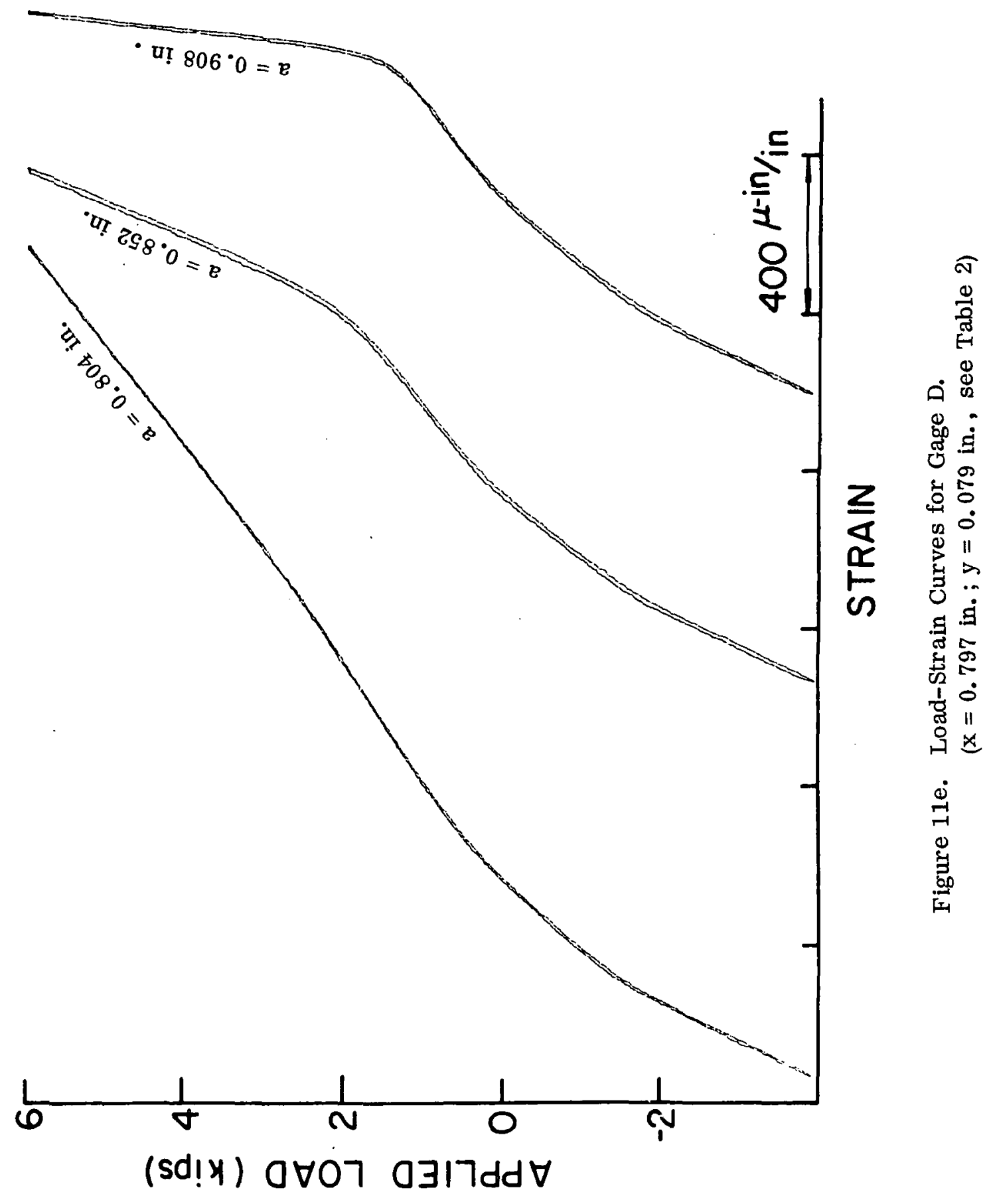




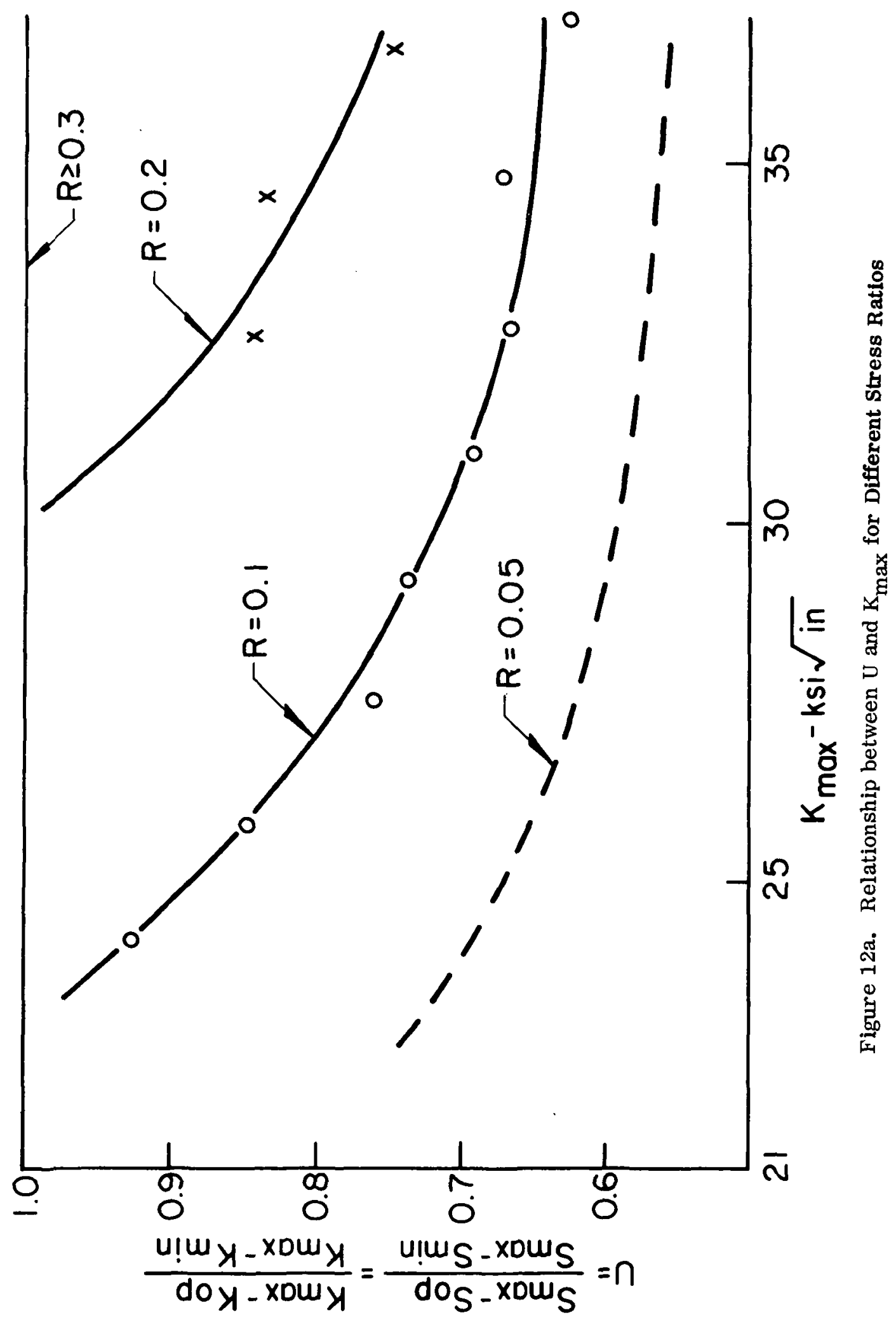




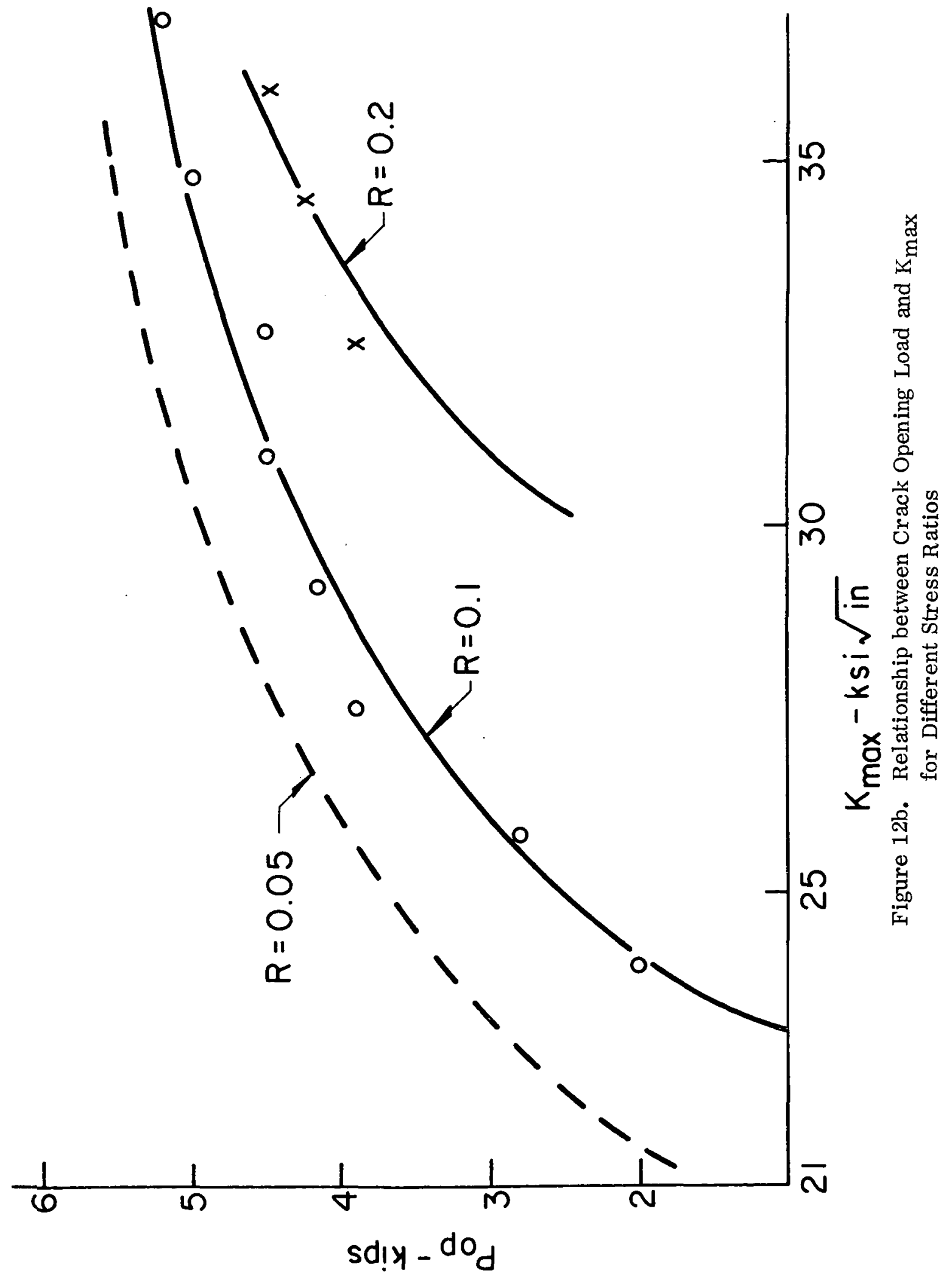



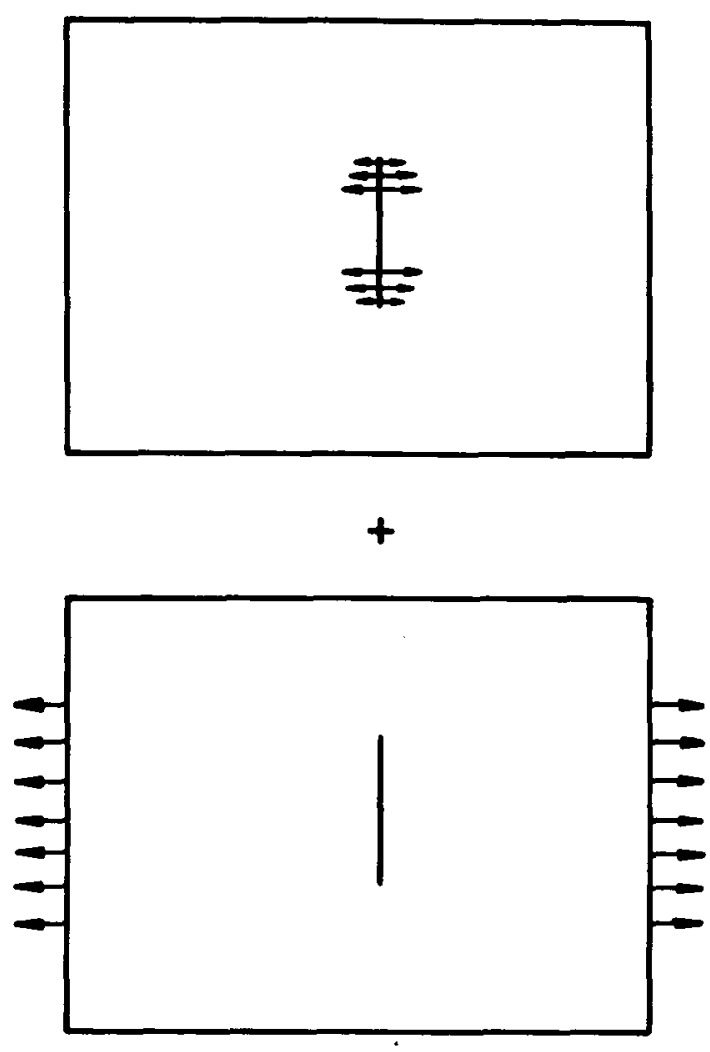

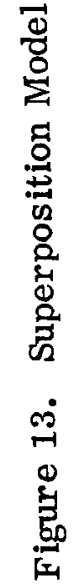

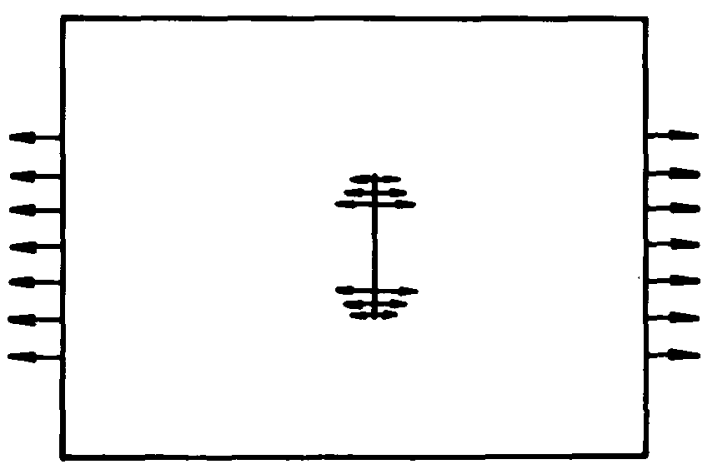




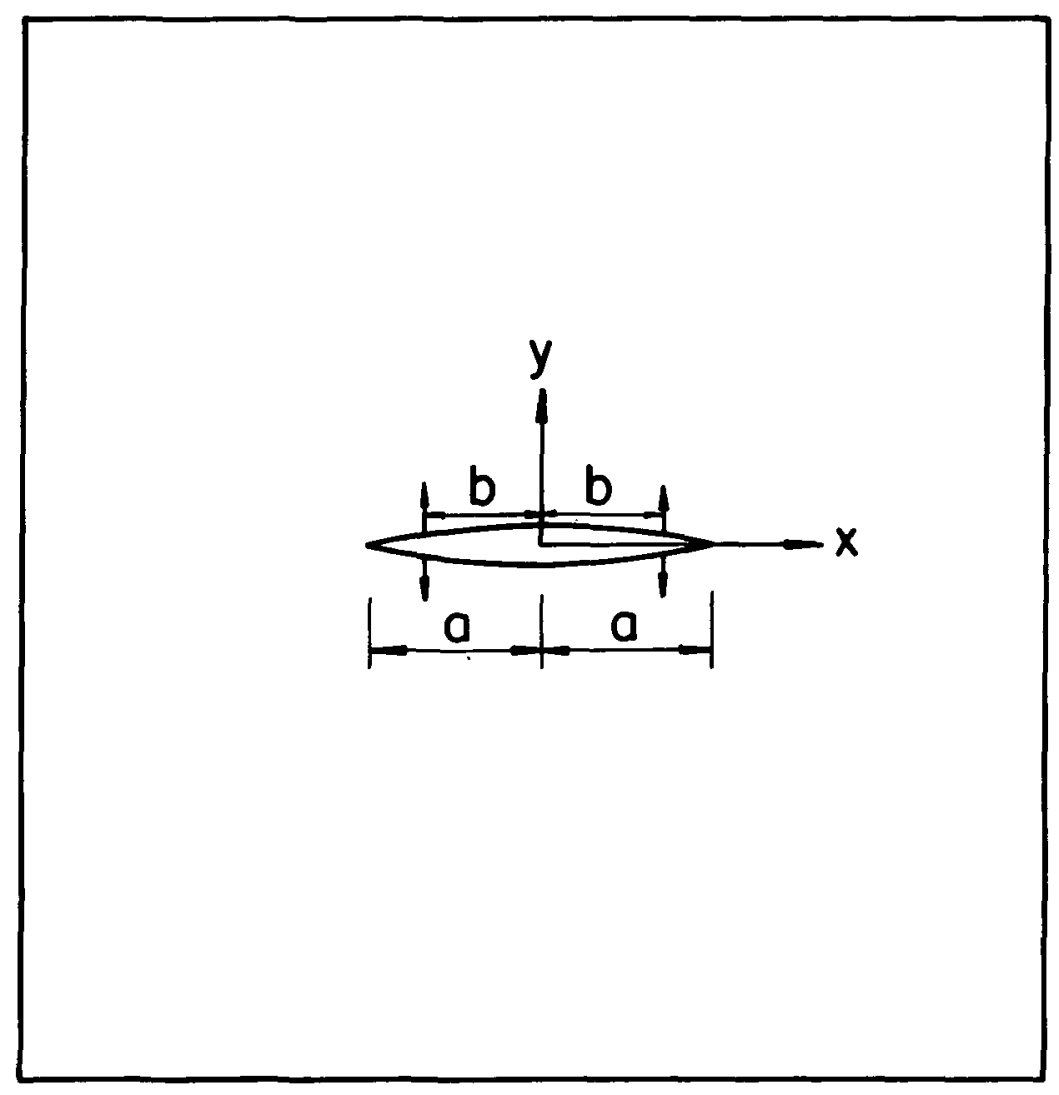

Figure 14. Central Crack of Length $2 \mathrm{a}$ in an Infinite Plate with Equal Pairs of Splitting Forces at $x= \pm b$ 


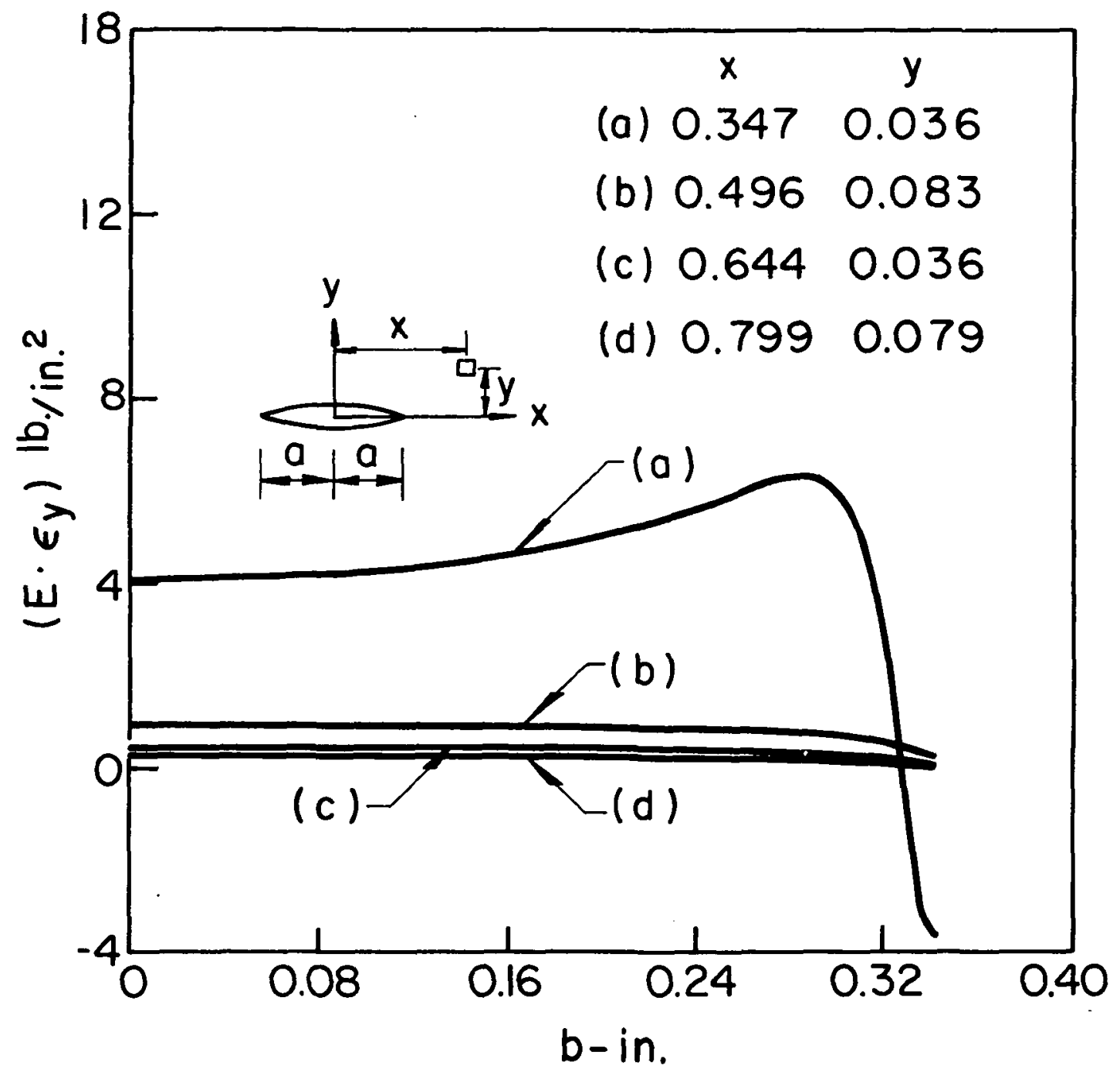

Figure 15. Relationships between Strains and the Position of Unit Splitting Forces with Strain Gages Located Ahead of the Crack Tip ( $a=0.34$ in.) 


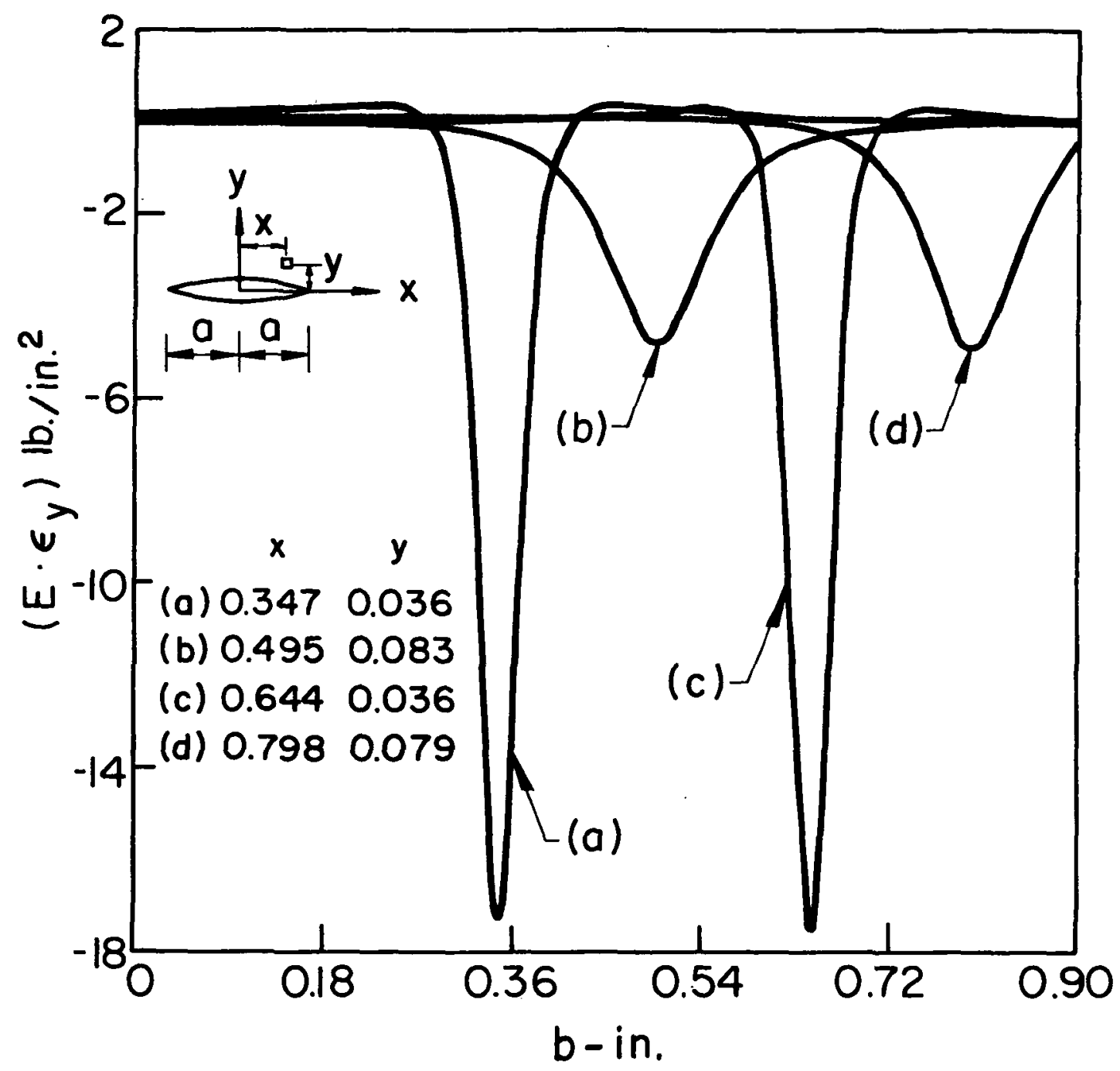

Figure 16. Relationships between Strains and the Position of Unit Splitting Forces with Strain Gage Located Behind the Crack Tip $(a=0.908$ in. $)$ 


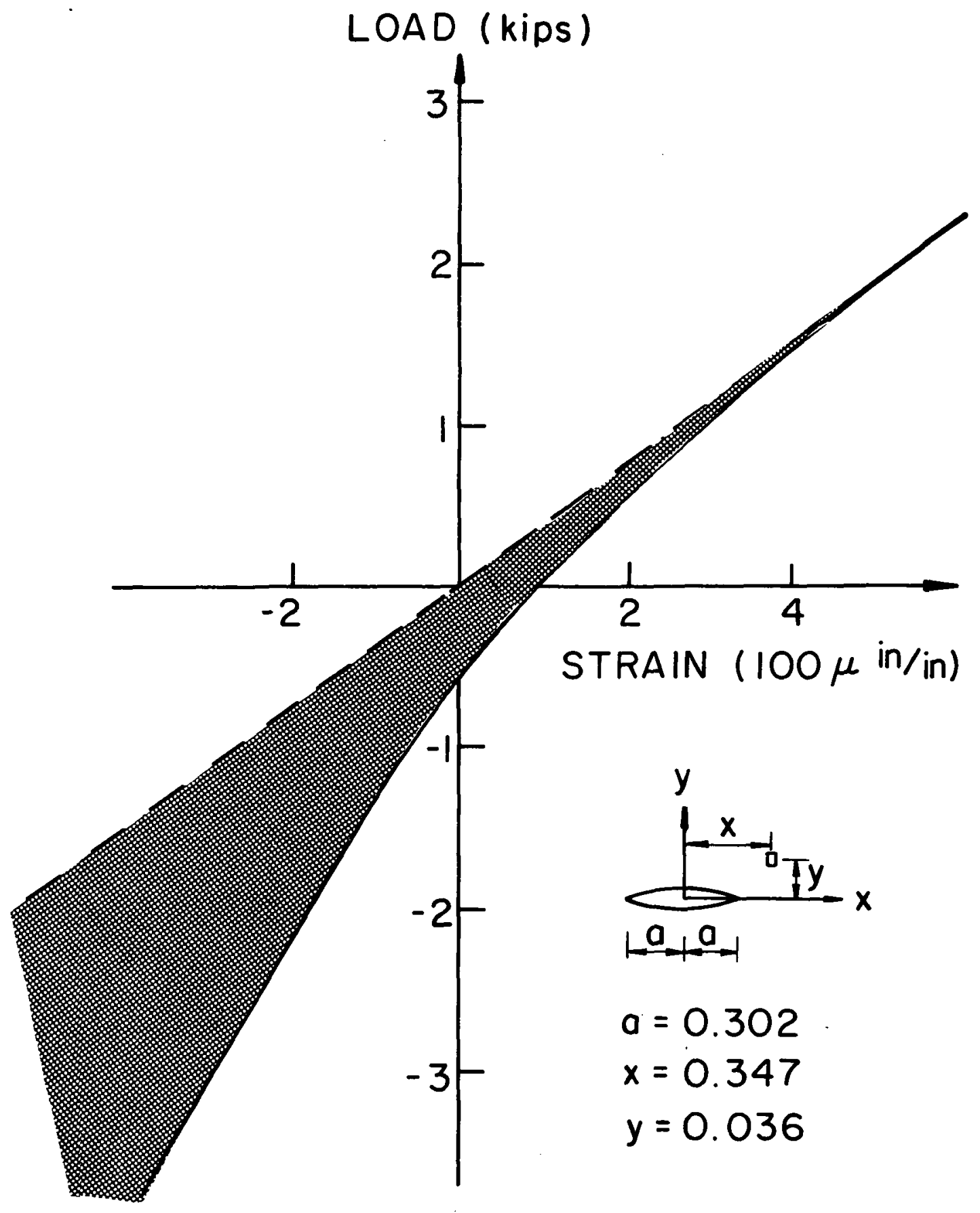

Figure 17. Typical Load versus Strain Curve for a Strain Gage Located Ahead of the Crack Tip 


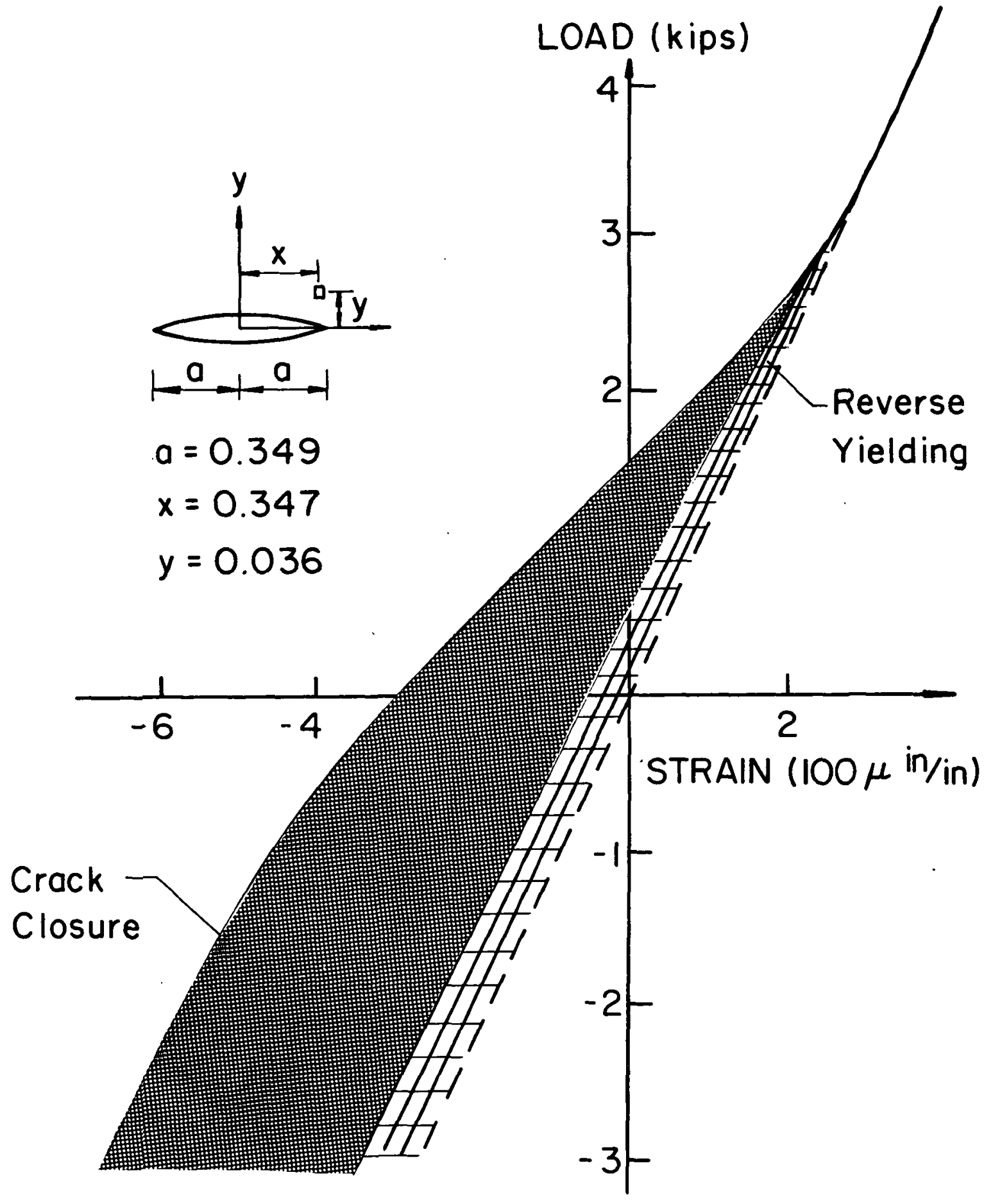

Figure 18. Typical Load versus Strain Curve for a Strain Gage Located Just Behind the Crack Tip 


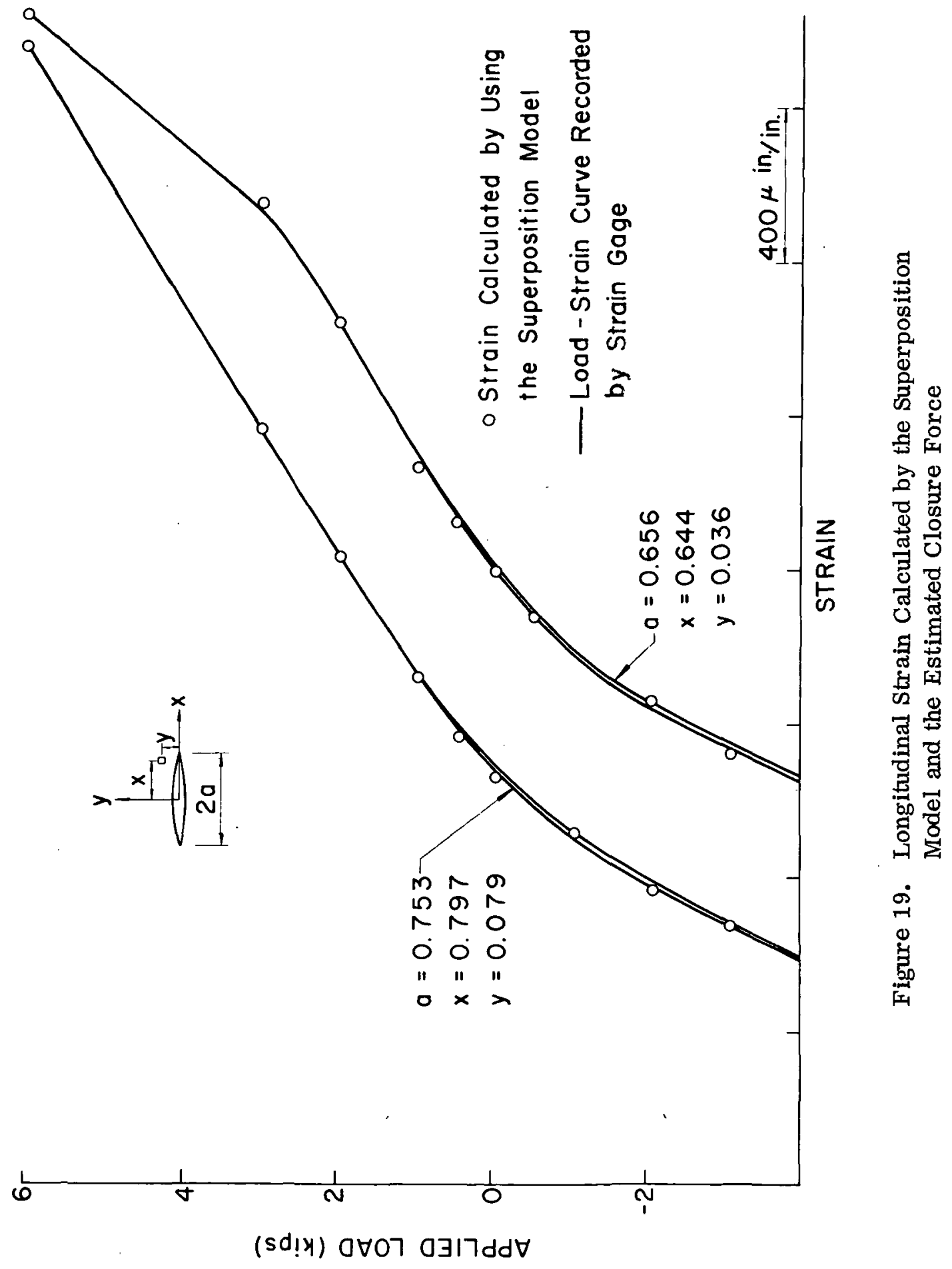




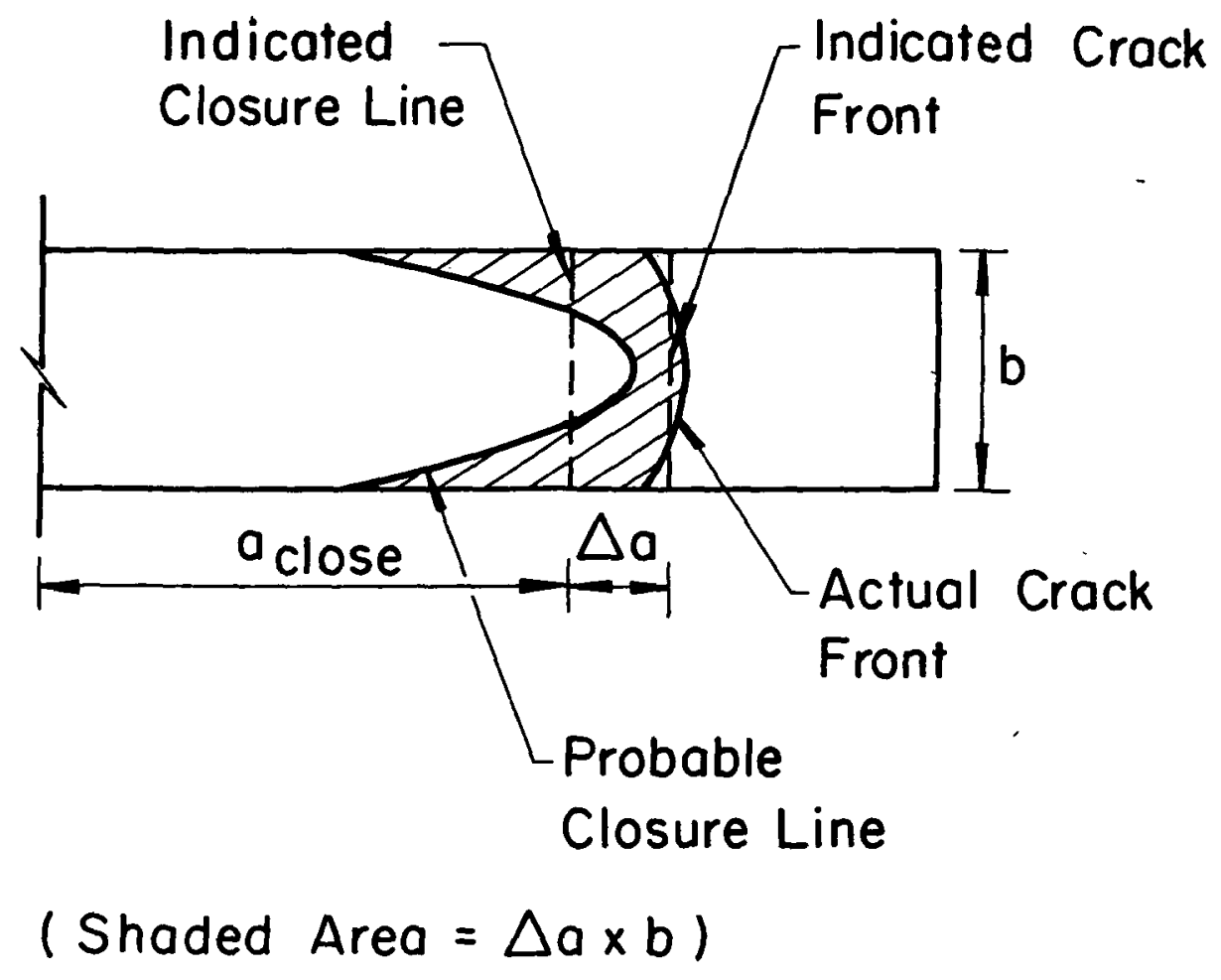

Figure 20. Comparison of Probable Closure Line with Closure Line Indicated by the Electrical Potential Measurements 


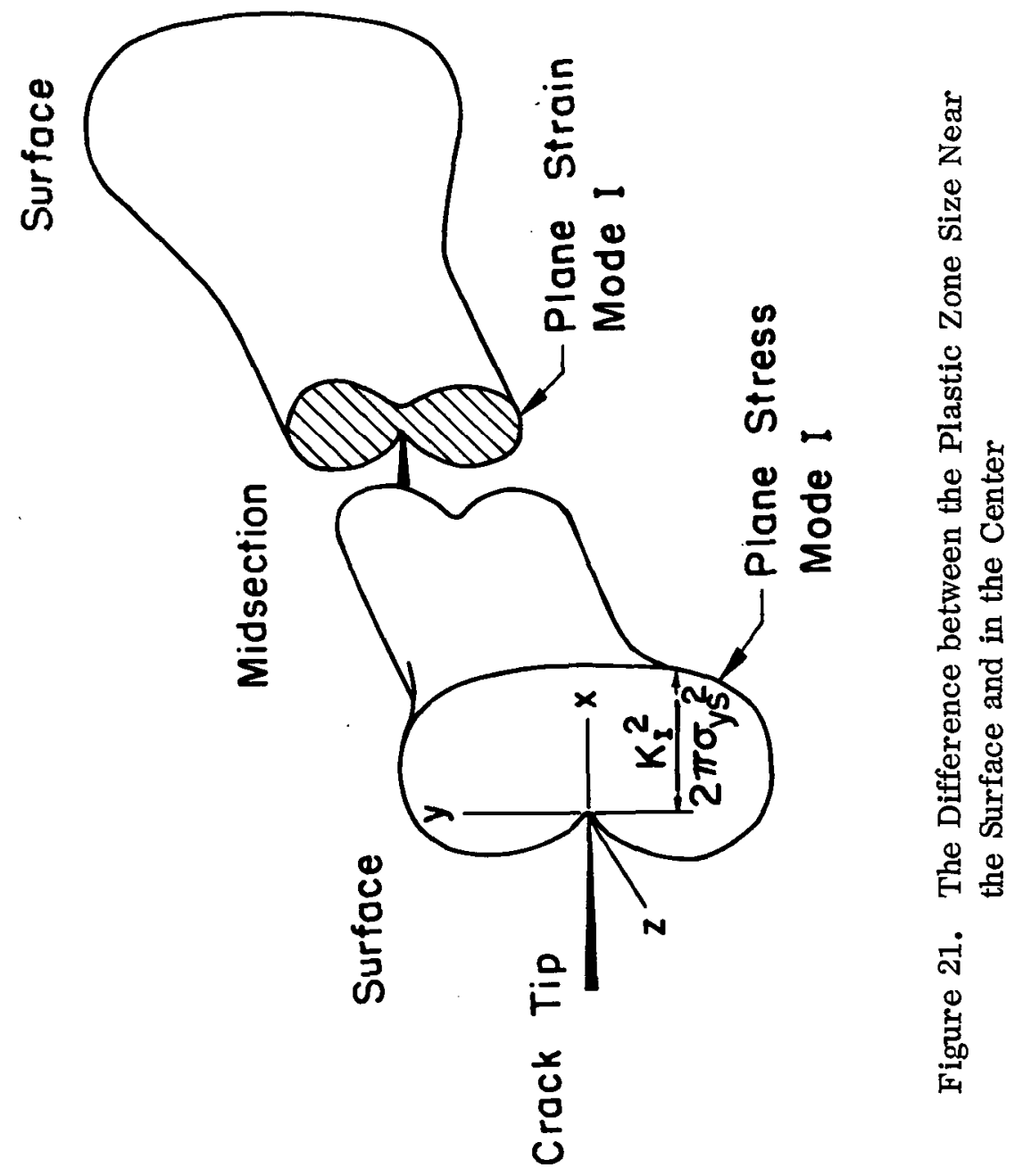




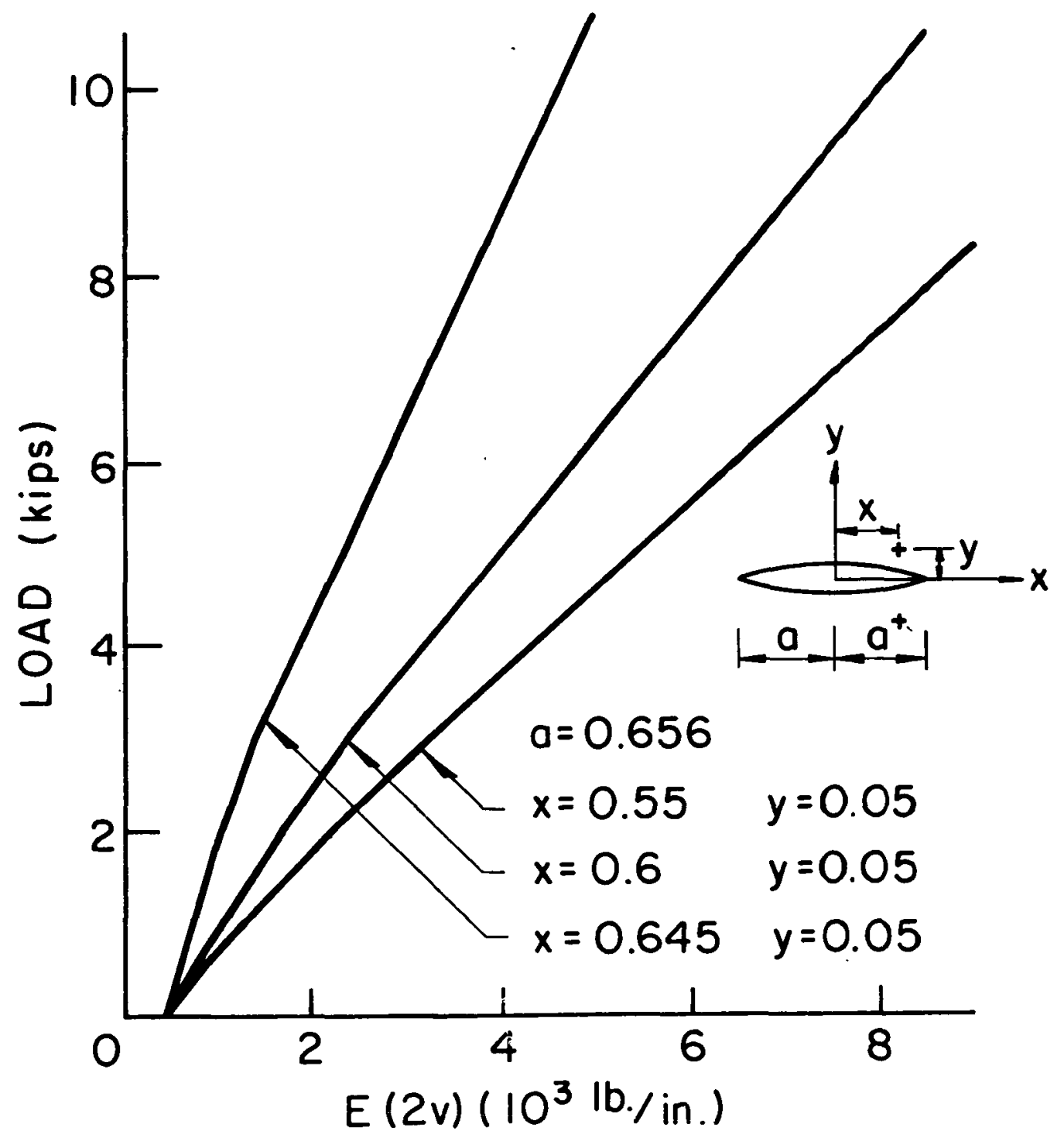

Figure 22. Relationship between Load and Relative Displacement between Gage Points Computed by Using the Superposition Model 

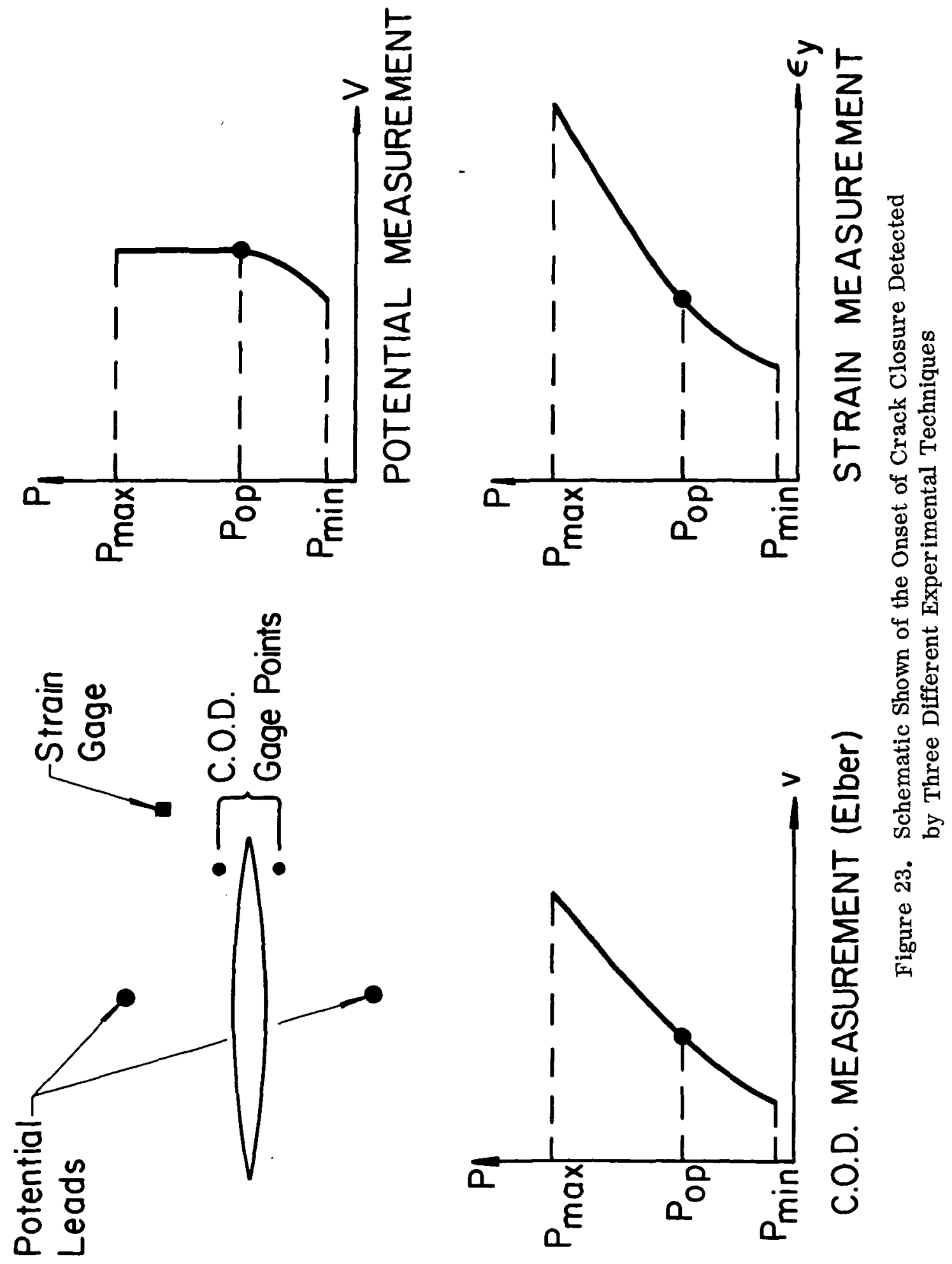


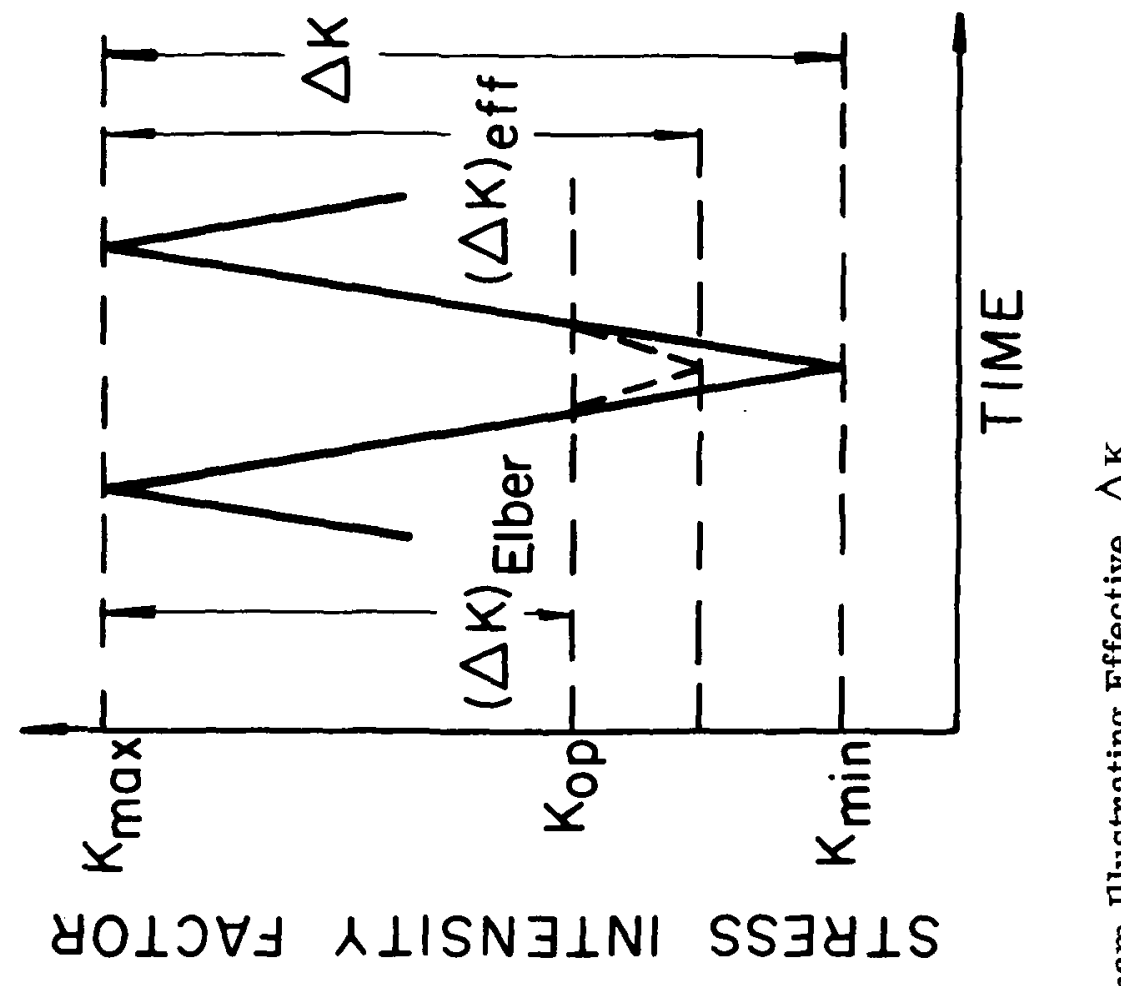

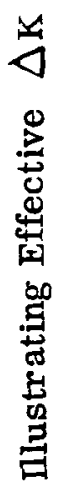

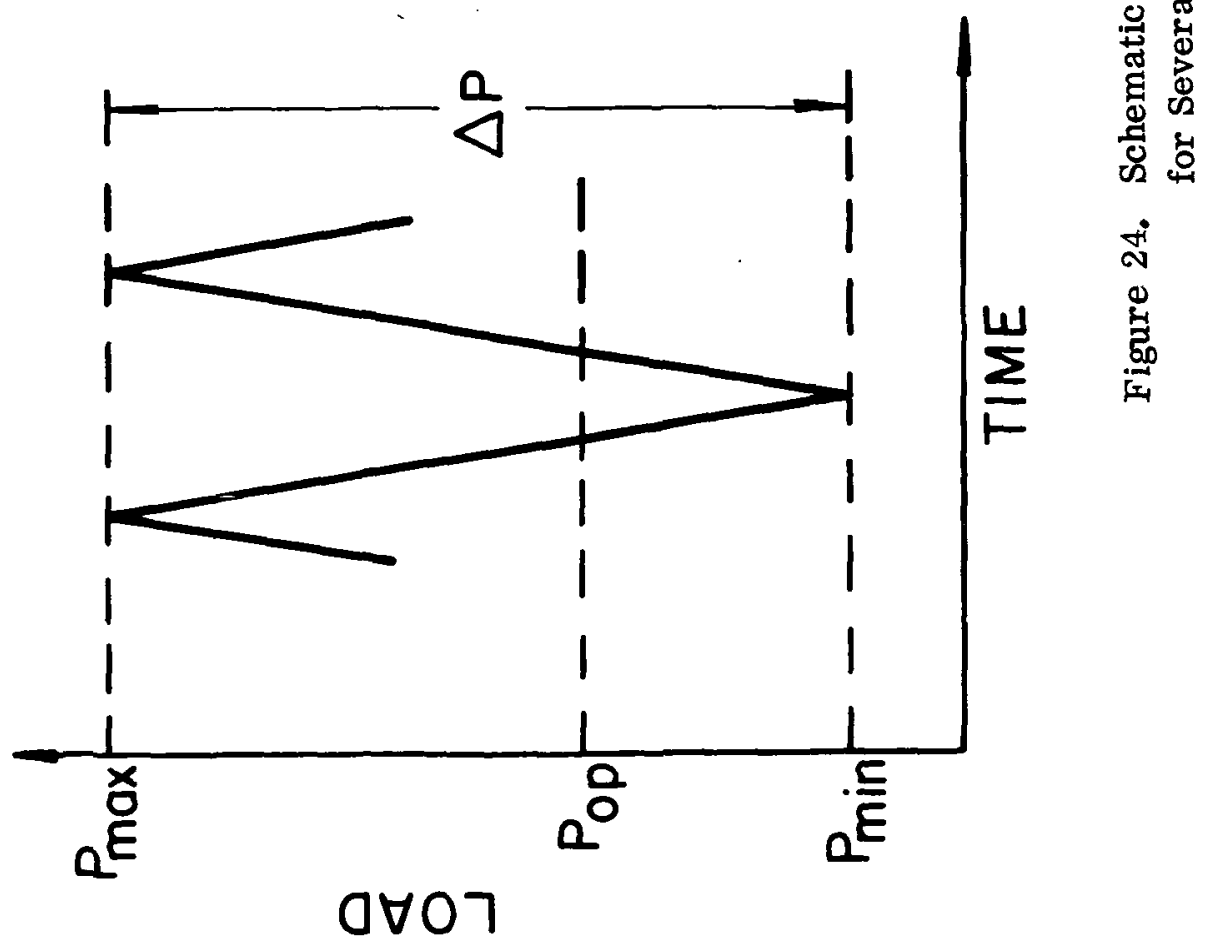




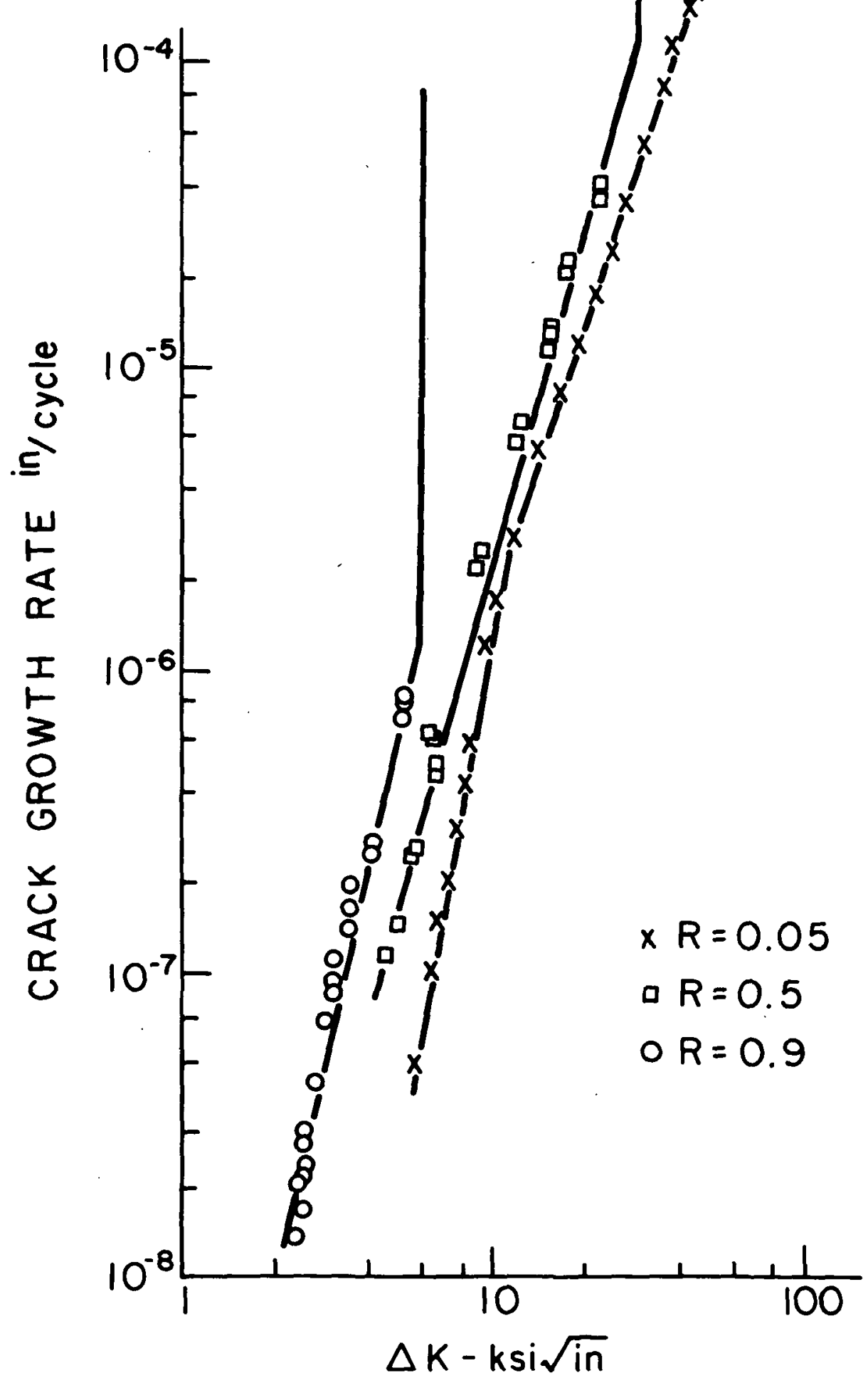

Figure 25. Crack Growth Rate for Ti-6Al-4V Alloy 


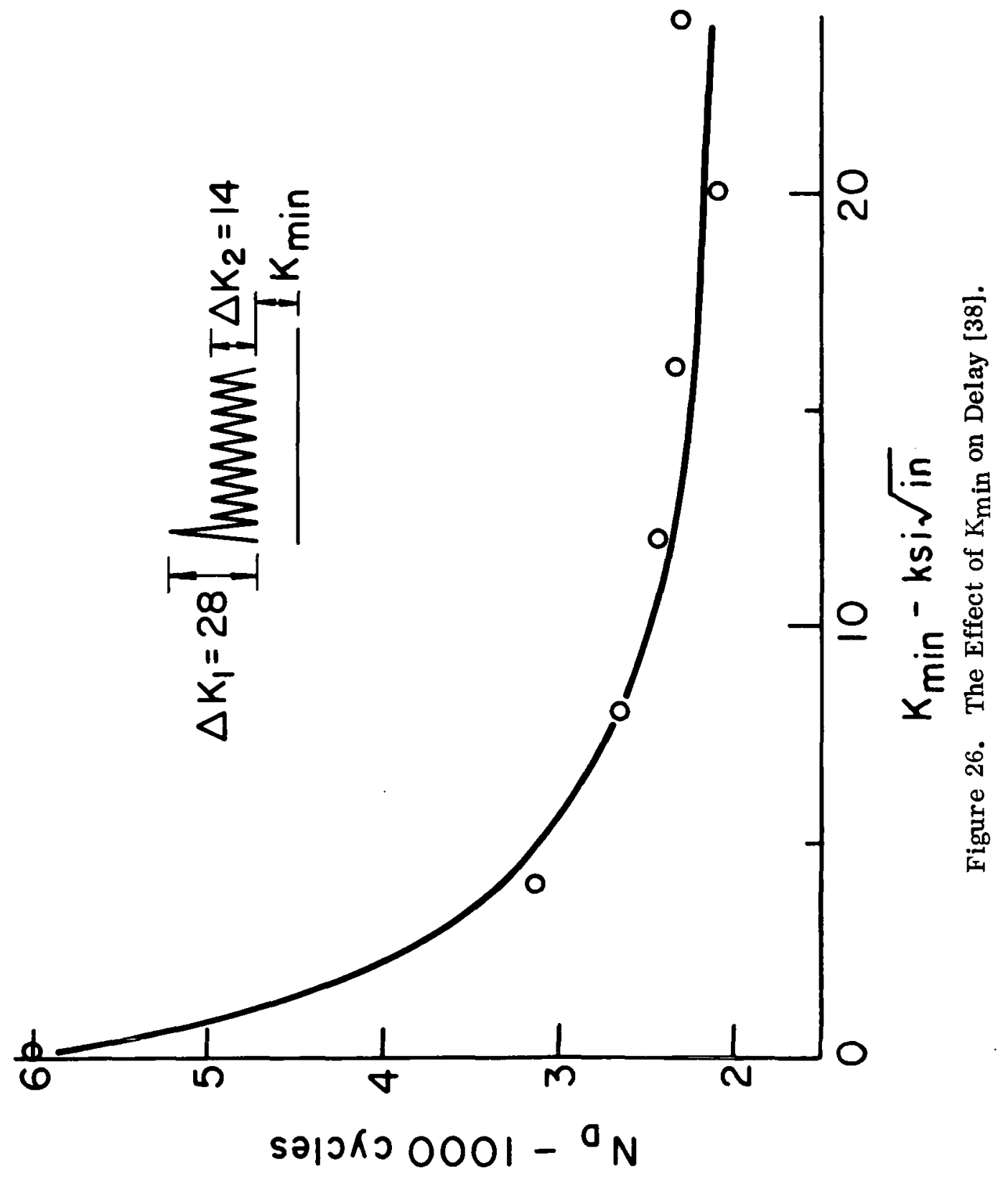




\section{REFERENCES}

1. H. F. Hardrath, "Fatigue and Fracture Mechanics," AIAA paper No. 70-512, April 1970.

2. P. C. Paris, "The Fracture Mechanics Approach to Fatigue", Proc. 10th Sagamore Conf., Syracuse University Press (1965).

3. S. R. Swanson, F. Cicci and W. Hoppe, ASTM 416 (1968).

4. H. H. Johnson and P. C. Paris, J. of Eng. Fracture Mech. , 1, (1968)

5. D. Broek and J. Schijve, "The Influence of the Mean Stress on the Propagation of Fatigue Cracks in Aluminum Alloy Sheet," NLR-Tech. Rep. M 2111 (1963).

6. R. G. Forman, V. E. Kearney, and R. M. Engle, "Numerical Analysis of Crack Propagation in Cyclically Loaded Structures, " J. of Basic Eng. , 89, 3 (Sept. 1967).

7. P. C. Paris, M. P. Gomez and W. E. Anderson, "A Rational Analytic Theory of Fatigue," The Trend in Engineering, 13, 9, (1961).

8. P. C. Paris and F. Erdogan, J. of Basic Engineering, Trans. ASME, 1963.

9. C. M. Hudson and J. T. Scardina, Eng. Fracture Mech., No. 1, (1969).

10. W. Elber, ASTM STP 486 (1971).

11. O. Buck, C. L. Ho, H. L. Marcus and R. B. Thompson, ASTM STP 513, (1972).

12. O. Buck, C. L. Ho and H. L. Marcus, reported in "The Symposium on Fracture and Fatigue," Washington, D. C. (1972).

13. C. L. Ho, O. Buck and H. L. Marcus, "Application of Strip Model to Crack Tip Resistance andCrack Closure Phenomena, "presented at Sixth National Symposium on Fracture Mechanics, Philadelphia, Aug. 1972.

14. R. A. Schmidt, and P. C. Paris, "Threshold for Fatigue Crack Propagation and the Effects of Load Ratio and Frequency, "presented at Sixth Annual Fracture Mechanics Symposium, Philadelphia, Aug. 1972. 
15. M. Katcher and M. Kaplan, "Effect of R Factor and Crack Closure on Aluminum and Titanium Alloys," presented at Sixth Annual Fracture Mechanics Symposium, Philadelphia, Aug. 1972.

16. V. W. Trebules, R. Roberts, and R. W. Hertzberg, "The Effect of Multiple Overload on Fatigue Crack Propagation in 2024-T3 Aluminum Alloy, " presented at Sixth Annual Fracture Mechanics Symposium, Philadelphia, Aug. 1972.

17. R. W. Hertzberg and E. F. J. Von Euw, "Crack Closure and Fatigue Striations," Met. Trans. $\underline{4}, 887$ (1973).

18. E. F. J. Von Euw, "Effect of Overload Cycle(s) on Subsequent Fatigue Crack Propagation in 2024-T3 Aluminum Alloy," Ph. D. dissertation, Lehigh University (1971).

19. W. F. Brown, Jr. and J. E. Srawley, ASTM STP 410 (1966).

20. Is ida, "Crack Tip Stress Intensity Factor for the Tension of an Eccentrically Cracked Strip," Lehigh University, Dep. of Mech. Rep. (1965). (see Reference 19)

21. H. H. Johnson, Materials Research and Standards, 5 (1965).

22. C. Y. LI and R. P. Wei, Materials Research and Standards, 6 (1966).

23. R. P. Wei and D. L. Ritter, J. of Materials, ASTM (June 1972).

24. P. C. Paris and G. C. Sih, ASTM STP 381 (1965).

25. F. A. McClintock and G. R. Erwin, ASTM STP 381 (1965).

26. J. M. Krafft, Trans. ASM, $\underline{58}, 691$ (1965).

27. J. M. Krafft, (discussion) ASTM STP 415 (1967).

28. R. P. Wei and T. T. Shih, "Delay in Fatigue Crack Growth"' Int'l J. of Fracture Mechanics (in press).

29. D. S. Dugdale, J. Mech. Phys. Solids, 8 (1960).

30. J. R. Rice, ASTM STP 415 (1967)

31. J. FitzGerald and R. P. Wei, to be published by ASTM (copies are available from the authors).

32. R. H. Christensen, Proceedings - Crack Propagation Symposium, Cranfield, College of Aeronautics (1962). 
33. J. Schijve, Fatigue Crack Propagation in Light Alloy Sheet Material and Structures, Rept. MP 195, National Luchtvaartlaboratorium (Amsterdam) (August 1960).

34. C. M. Hudson and H. F. Hardrath, NASA TN D-960 (1961).

35. O. Jonas and R. P. Wei, Int'l. J. Fract. Mech., 7 (1971), 116-118.

36. C. M. Hudson and H. F. Hardrath, "Investigation of Variable Loading on Fatigue Crack Propagation, "NASA Tech. Note D-1803 (1965). 


\section{APPENDIX}

The equation of equilibrium for two dimensional elastic problems can be found in any elementary text of the theory of elasticity.

$$
\begin{aligned}
& \frac{\partial \sigma_{x}}{\partial x}+\frac{\partial \tau_{x y}}{\partial y}=0 \\
& \frac{\partial \tau_{x y}}{\partial x}+\frac{\partial \sigma_{y}}{\partial y}=0 \\
& \tau_{x y}=\tau_{y x}
\end{aligned}
$$

The strain-displacement relationships and Hook's law lead to the compatibility equation.

$$
\left(\frac{\partial^{2}}{\partial \chi^{2}}+\frac{\partial^{2}}{\partial y^{2}}\right)\left(\sigma_{x}+\sigma_{y}\right)=0
$$

As is easily checked, equations A-1 are satisfied by an Airy's stress function, $\Phi(x, y)$, if the stress components are defined as follows:

$$
\begin{aligned}
& \sigma_{x}=\frac{\partial^{2} \Phi}{\partial y^{2}} \\
& \sigma_{y}=\frac{\partial^{2} \Phi}{\partial x^{2}} \\
& \tau_{x y}=-\frac{\partial^{2} \Phi}{\partial x \partial y}
\end{aligned}
$$

Substitutions of Equation A-3 into Equation A-2 gives the biharmonic equation in 玉.

$$
\nabla^{4} \Phi=\frac{\partial^{4} \Phi}{\partial x^{4}}+2 \frac{\partial^{4} \Phi}{\partial x^{2} \partial y^{2}}+\frac{\partial^{4} \Phi}{\partial y^{4}}=0
$$

For a specific problem, the stress function, $\Phi$, must satisfy Equation A-4 and the appropriate boundary conditons. Theory of functions of complex variables provides a powerful tool for the solution of two-dimensional elasticity problems. 
Define a complex variable, $z$, by

$$
z=x+i y
$$

If a function of this complex variable, $\overline{\overline{\mathrm{Z}}}(\mathrm{z})$, is analytic then its derivatives exist, that is,

$$
\begin{aligned}
& \widetilde{Z}=\frac{d \overline{\bar{Z}}}{d z} \\
& Z=\frac{d \bar{Z}}{d z} \\
& Z^{\prime}=\frac{d z}{d z}
\end{aligned}
$$

Let $\mathrm{Z}=\operatorname{Re} \mathrm{Z}+\mathrm{i} \operatorname{Im} \mathrm{Z}$, then the Cauchy Rieman conditions state that:

$$
\begin{aligned}
& \frac{\partial}{\partial x} \operatorname{Re} \overline{\overline{\mathrm{z}}}=\frac{\partial}{\partial y} \operatorname{Im} \overline{\overline{\mathrm{z}}}=\operatorname{Re} \overline{\mathrm{z}} \\
& \frac{\partial}{\partial \chi} \operatorname{Im} \overline{\overline{\mathrm{Z}}}=-\frac{\partial}{\partial y} \operatorname{Re} \overline{\overline{\mathrm{Z}}}=\operatorname{Im} \overline{\mathrm{Z}}
\end{aligned}
$$

It follows that

$$
\nabla^{2}(\operatorname{Re} \overline{\bar{Z}})=\nabla^{2}(\operatorname{Im} \overline{\bar{Z}})=0
$$

Similarly, all derivatives of $\mathrm{Z}$ have harmonic real and imaginary parts. If the $\psi_{i}$ are each harmonic, that is

$$
\nabla^{2} \psi_{i}=0
$$

then any stress function in the form

$$
\Phi=\psi_{1}+x \psi_{2}+y \psi_{3}+\left(x^{2}+y^{2}\right) \psi_{4}
$$

will automatically satisfy the Equation A-4.

In conformity with Equations A-5 - A-9, Westgaard defined an Airy stress function, $\Phi$, by 


$$
\Phi=\operatorname{Re} \overline{\overline{\mathrm{Z}}}+\mathrm{y} \operatorname{Im} \overline{\mathrm{Z}}
$$

This function satisfies the equilibrium Equations, A-1, and the compatibility condition, Equation A-2.

Using Equation A-3, A-7, and A-10 the stress are defined by Equation A-11:

$$
\begin{aligned}
& \sigma_{x}=\operatorname{Re} z-y \operatorname{Im} z^{\prime} \\
& \sigma_{y}=\operatorname{Re} z+y \operatorname{Im} z^{\prime} \\
& \tau_{x y}=-y \operatorname{Re} z^{\prime}
\end{aligned}
$$

The displacement-strain relations are given by Equation A-12:

$$
\begin{aligned}
& \frac{\partial u}{\partial x}=\epsilon_{x} \\
& \frac{\partial v}{\partial y}=\epsilon_{y}
\end{aligned}
$$

The strain-stress relations for generalized plane stress condition are given by Equation A-13

$$
\begin{aligned}
& E \epsilon_{x}=\sigma_{x}-\nu \dot{\sigma}_{y} \\
& E \epsilon_{y}=\sigma_{y}-\nu \sigma_{x}
\end{aligned}
$$

Combining Equation A-11, A-12, and A-13, the in-plane displacements and strains are obtained.

$$
\begin{aligned}
& E_{\mathfrak{u}}=(1-\nu) \operatorname{Re} \overline{\mathrm{Z}}-(1+\nu) y \operatorname{Im} \mathrm{Z} \\
& E_{\mathrm{V}}=2 \operatorname{Im} \overline{\mathrm{Z}}-(1+\nu) \text { y } \operatorname{Re} \mathrm{Z}
\end{aligned}
$$




$$
\begin{aligned}
& E E_{x}=(1-\nu) \operatorname{Re} Z-(1+\nu) y \operatorname{Im} Z^{\prime} \\
& E E_{y}=(1-\nu) \operatorname{Re} Z+(1+\nu) y \operatorname{Im} Z^{\prime}
\end{aligned}
$$

For a central crack of length $2 a$ in an infinite plate with uniform biaxial stress 6 at infinity, the stress function is given by Equations A-16 [24]:

$$
\begin{aligned}
& \bar{z}=\sigma \sqrt{z^{2}-a^{2}} \\
& z=\sigma z / \sqrt{z^{2}-a^{2}} \\
& z^{\prime}=\sigma / \sqrt{z^{2}-a^{2}}-\sigma z^{2} / \sqrt{\left(z^{2}-a^{2}\right)^{3}}
\end{aligned}
$$

The longitudinal strain and displacement at a point $A(x, y)$ are then obtained from Equations A-14 and A-15.

$$
\begin{aligned}
& \mathrm{E} \epsilon_{\mathrm{y}}=\sigma\left[(1-y) \operatorname{Re}\left(\frac{\mathrm{z}}{\sqrt{\mathrm{z}^{2}-\mathrm{a}^{2}}}\right)+(1+\nu) y \operatorname{Im}\left(\frac{-\mathrm{a}^{2}}{\sqrt{\left(\mathrm{z}^{2}-\mathrm{a}^{2}\right)^{3}}}\right)\right] \\
& \mathrm{Ev}=\sigma\left[2 \operatorname{Im}\left(\sqrt{\mathrm{z}^{2}-\mathrm{a}^{2}}\right)-(1+\nu) \text { y } \operatorname{Re}\left(\frac{\mathrm{z}}{\sqrt{\mathrm{z}^{2}-\mathrm{a}^{2}}}\right)\right]
\end{aligned}
$$

The stress function for a central crack with two equal pairs of splitting forces at $\mathrm{x}= \pm \mathrm{b}$ (Figure 14) is given by Equations $\mathrm{A}-18$.

$$
\begin{aligned}
& \bar{Z}=\frac{2 P}{\pi} \tan ^{-1} \sqrt{\frac{z^{2}-a^{2}}{a^{2}-b^{2}}} \\
& \mathrm{Z}=\frac{2 \mathrm{P}}{\pi} \frac{\mathrm{z} \sqrt{\mathrm{a}^{2}-\mathrm{b}^{2}}}{\left(z^{2}-\mathrm{b}^{2}\right) \sqrt{\mathrm{z}^{2}-\mathrm{a}^{2}}} \\
& \mathrm{Z}^{\prime}=-\frac{2 \mathrm{P}}{\pi}\left[\frac{\mathrm{a}^{2} \sqrt{\mathrm{a}^{2}-\mathrm{b}^{2}}}{\left(\mathrm{z}^{2}-\mathrm{b}^{2}\right) \sqrt{\left(z^{2}-a^{2}\right)^{3}}}+\frac{2 \mathrm{z}^{2} \sqrt{\mathrm{a}^{2}-\mathrm{b}^{2}}}{\left(\mathrm{z}^{2}-\mathrm{b}^{2}\right)^{2} \sqrt{\mathrm{z}^{2}-\mathrm{a}^{2}}}\right]
\end{aligned}
$$


The longitudinal strain and displacement at a point $\mathrm{A}(\mathrm{x}, \mathrm{y})$ is then given by Equations A-19.

$$
\begin{aligned}
E \epsilon_{y} & =\frac{2 P}{\pi} \cdot(1-\nu) \operatorname{Re}\left(\frac{z \sqrt{a^{2}-b^{2}}}{\left(z^{2}-b^{2}\right) \sqrt{z^{2}-a^{2}}}\right)-(1+\nu) y \operatorname{Im}\left(\frac{a^{2} \sqrt{a^{2}-b^{2}}}{\left(z^{2}-b^{2}\right) \sqrt{\left(z^{2}-a^{2}\right)^{3}}}\right. \\
+ & \left.\left.\frac{2 z^{2} \sqrt{a^{2}-b^{2}}}{\left(z^{2}-b^{2}\right)^{2} \sqrt{z^{2}-a^{2}}}\right)\right] \\
E v & =\frac{2 P}{\pi}\left[2 \operatorname{Im}\left(\tan ^{-1}\left(\sqrt{\frac{z^{2}-a^{2}}{a^{2}-b^{2}}}\right)-(1+\nu) \text { y } \operatorname{Re}\left(\frac{z \sqrt{a^{2}-b^{2}}}{\left(z^{2}-b^{2}\right) \sqrt{z^{2}-a^{2}}}\right)\right)\right.
\end{aligned}
$$


"The aeronautical and space activities of the United States shall be conducted so as to contribute ... to the expansion of buman knowledge of phenomena in the atmosphere and space. The Administration shall provide for the widest practicable and appropriate dissemination of information concerning its activities and the results thereof."

-National Aeronautics and Space ACt of 1958

\section{NASA SCIENTIFIC AND TECHNICAL PUBLICATIONS}

TECHNICAL REPORTS: Scientific and technical information considered important, complete, and a lasting contribution to existing knowledge.

TECHNICAL NOTES: Information less broad in scope but nevertheless of importance as a contribution to existing knowledge.

\section{TECHNICAL MEMORANDUMS:}

Information receiving limited distribution because of preliminary data, security classification, or other reasons. Also includes conference proceedings with either limited or unlimited distribution.

CONTRACTOR REPORTS: Scientific and technical information generated under a NASA contract or grant and considered an important contribution to existing knowledge.
TECHNICAL TRANSLATIONS: Information published in a foreign language considered to merit NASA distribution in English.

SPECIAL PUBLICATIONS: Information derived from or of value to NASA activities. Publications include final reports of major projects, monographs, data compilations, handbooks, sourcebooks, and special bibliographies.

\section{TECHNOLOGY UTILIZATION} PUBLICATIONS: Information on technology used by NASA that may be of particular interest in commercial and other non-aerospace applications. Publications inciude Tech Briefs, Technology Utilization Reports and Technology Surveys.

Defails on the availability of these publications may be obtained from:

SCIENTIFIC AND TECHNICAL INFORMATION OFFICE 